\title{
AN ANALYSIS OF LANTHANUM SPECTRA (LA I, LA II, LA III)
}

\author{
By Henry Norris Russell ${ }^{1}$ and William F. Meggers
}

\section{ABSTRACT}

All the available data (wave-length measurements and intensity estimates, temperature classes, Zeeman effects) on lanthanum lines have been correlated and interpreted in an analysis of the successive optical spectra. The total number of lines classified is 540 in the La I spectrum, 728 in the La II spectrum, and 10 in the La III spectrum.

Series-forming terms have been identified in each spectrum and from these the ionization potentials of 5.59 volts for neutral La atoms, 11.38 volts for Lat atoms and 19.1 volts for $\mathrm{La}^{++}$atoms have been deduced.

Lanthanum is a chemical analogue of scandium and yttrium, but, although the corresponding spectra are strikingly similar, some interesting differences are noted. A doublet-D term (from a $\bar{d}$ electron) represents the lowest energy (normal state) in the third spectrum of each element and another ${ }^{2} \mathrm{D}$ (from the $s^{2} d$ configuration) describes the normal state of the neutral atoms in each case. The homologous atoms $\mathrm{Sc}^{+}, \mathrm{Y}^{+}, \mathrm{La}^{+}$choose different normal states; $(s d){ }^{3} \mathrm{D}$, $\left(s^{2}\right){ }^{1} \mathrm{~S},\left(d^{2}\right) 3 \mathrm{~F}$, respectively. In addition, the first two spectra of La exhibit a large number of (odd) middle-set terms ascribed to the binding of an $f$ electron. The La II spectrum is the most completely developed example of a two-electron spectrum which has yet been investigated. All the configuration types, $s^{2}, s p, s d$, $s f, p^{2}, p d, p f, d^{2}, d f, f^{2}$, have been identified and almost all of the terms arising from each.

The analyses of all three spectra are supported by measurements of Zeeman effects, which are interpreted with the aid of Landé's theory. The splitting factors ( $g$ values) for many levels show marked departure from the theoretical values, but the " $g$-sum rule" is valid wherever it is tested.

\section{CONTENTS}

I. Introduction

II. The spectrum of doubly ionized lanthanum (La III)

III. The spectrum of singly ionized lanthanum (La II)

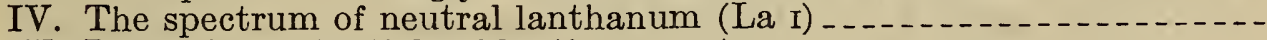

V. Ionization potentials of lanthanum atoms

VI. Discussion of Zeeman effects

665

\section{INTRODUCTION}

After the analyses of the arc and spark spectra of scandium ${ }^{2}$ (Sc, $\mathrm{Z}=21$ ) and yttrium ${ }^{3}(\mathrm{Y}, \mathrm{Z}=39)$ had been published the authors decided to continue cooperation on the remaining spectra of this type. Lanthanum ( $\mathrm{La}, \mathrm{Z}=57$ ) is a chemical analogue of scandium and yttrium; it occupies the same position in the third long period of elements that the former do in the first and second long periods, respectively. The fact that lanthanum occupies a position in the periodic system just preceding the group of 14 elements commonly called "rare earths" makes a complete analysis of its spectra of exceptional interest. Indeed, this analysis shows (vide infra) that the

1 Professor of astronomy, Princeton University.

2 H. N. Russell and W. F. Meggers, B. S. Sci. Paper No. 558, vol. 22, p. 329, 1927

3 W. F. Meggers and H. N. Russell, B. S. Jour, Research, vol, 2 (RP55), p. 733, 1929. 
atom building process which accounts for the rare earth elements is actually anticipated in the electron configurations of the lanthanum atom. Furthermore, the exact nature of electron coupling in complex atoms like $\mathrm{La}$, as disclosed by the analysis of spectral structure, is certain to be important in the further development of theory. In the present paper the authors give the results of an extensive analysis of lanthanum spectra in practically the same form as their results for scandium and yttrium; the summary of spectral theory given in the earlier publications applies also to the present case, and the notation is the same except for slight changes which bring it into conformity
with the standardized nomenclature ${ }^{4}$ and practice.

In 1914, Popow ${ }^{5}$ published the first suggestion of regularities in lanthanum spectra. From Zeeman effect observations, he recognized six La lines as comprising a combination of triplet $P$ and triplet D ferences occurringe year Paulson ${ }^{6}$ published a list of constant diflines, but no attemptween wave numbers corresponding to La spark earlier, Rybar ${ }^{7}$ had mas made to interpret the regularities. Even effects in La spectra and many compive measurements of the Zeeman there was at that time many complex patterns were published, but Another decade passed satisfactory explanation for most of these. began to unfold itself in the the theory of complex Zeeman effects an explanation of the work of Landé, ${ }^{8}$ thus paving the way for 1924 by Goudsmit ${ }^{9}$ La observations. An attempt was made in La II spectrum with who identified additional Paulson terms in the latter observations the aid of Rybar's Zeeman effects. From the $l$ were derived for 20 Landé $g$ values and quantum numbers $j$ and 70 lines.

In the following year the theory of spectral terms as developed by Heisenberg ${ }^{10}$ and by Hund ${ }^{11}$ gave some important suggestions as to the structures of La spectra and attempts were made by one of us to in the La I spectrum. These efforts succeeded also to find regularities of new empirical data consisting succeeded with the availability respect to their behavior with ten of a description of La lines with King and Carter ${ }^{12}$ and of unpublisherature in the electric furnace by by Prof. B. E. Moore. Th ublished Zeeman effects kindly advanced separation of I I I and The temperature classification gave a reliable effects, especially and La II lines, and the new observations of Zeeman clue to regula in the red portion of the spectrum, gave the first identified by Goudsmit, 22 I spectrum. In addition to the 20 levels binations of these 42 levels accounted found for $\mathrm{La}^{+}$atoms and comFor neutral La atoms 48 energy levels for about 180 La II lines. ${ }^{13}$ tions accounted for about 130 lines ${ }^{14}$ were found and their combinaor lowest energy levels were identified without cases the normal states spectrum many lines remained un without ambiguity, but in each terms were still undiscovered. Thelassified, and many theoretical

\footnotetext{
5 S. Popow, Ann. d. Physik, vol. 45, p. 147, 1914 . Turner, Phys. Rev., vol. 33, p. 900, 1929.

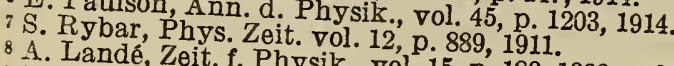

S. Goudsmit, Kon. A

10 W. Heisenberg, Zeit. f. Physik., vol. 32, 8 , vol. 33, No. 8, p. 774, 1921.

11 F. Hund, Zeit. f. Physik., Vol ., vol. 32, p. 841, 1925.

Julius, Springer, Berlin, 1927.
12 A. S. King and E. Carter, Astrophys. J., vol. 65, p. $86,1927$.

14 W. F. Meggers, J. Wt. Soc. Am., vol. 14, p. 191, 1927.
} 
that it would be impossible to extend them without still further experimental data, ${ }^{15}$ so it was decided to make an entirely new description of La spectra. Such a description was recently completed by one of us, ${ }^{16}$ and it serves as a basis for the analyses of La spectra to be detailed in the present paper. In addition to new wave-length determinations for more than 1,500 La lines in the interval 2,100 to $11,000 \mathrm{~A}$, the new data include intensity estimates of arc and spark lines, separating them into three classes, La I, La II, and La III spectra, and improved observations of Zeeman effects for 460 lines ranging from 2,700 to $7,500 \mathrm{~A}$. These data have permitted us to classify almost all of the lines ascribed to lanthanum atoms, to identify a large majority of the spectral terms, and correlate them with electron configurations. In each case it has been possible to recognize series-forming terms, the extrapolation of which lead to calculated ionization potentials of 5.59 volts for neutral La atoms, 11.38 volts for La ${ }^{+}$atoms, and 19.1 volts for $\mathrm{La}^{++}$atoms. The total numbers of classified lines in the successive spectra are as follows: 540 for La I, 728 for La II, and 10 for La III.

On account of the greater complexity of La spectra, as compared with $\mathrm{Sc}$ and $\mathrm{Y}$, and pronounced departures from theoretical interval ratios, line intensities and Zeeman effects, their analysis has been attended by greater difficulties and uncertainties, but patience and perseverance have been rewarded by the final classification of practically all lines without ambiguity. The detailed results will be presented for La III, then for La II, and finally for La I, thus proceeding from the relatively simple (alkali) case of 1 -valence electron to the 2-electron spectrum with greatly increased transition possibilities and lastly to the spectrum characteristics of atoms with a full complement of 3 -valence electrons.

\section{THE SPECTRUM OF DOUBLY IONIZED LANTHANUM (La III)}

Lanthanum belongs to the third long period in which electron orbits of the types $6 s, 6 p$, and $5 d$ are successively added to the completed xenon shell. Only one valence electron remains in doubly ionized lanthanum and in the normal state this electron is in a $5 d$ orbit which produces a $: D$ term. The next lowest state occurs with the $6 s$ orbit, and higher states arise from $6 p, 6 \bar{d}, 7 s$ orbits. Some of these terms were already identified by Gibbs and White ${ }^{17}$ and recently Badami ${ }^{18}$ classified eight lines of the La III spectrum.

The new description of La spectra yielded 10 lines which are characterized by an enormous intensity difference between arc and spark and are, therefore, ascribed to doubly ionized atoms. Analysis of these data resulted in the identification of spectral terms listed in Table 1; the observed lines and estimated relative intensities appear in Table 2.

The observed and theoretical (Landé) splitting factors $(g)$ are compared in the last column of the term table. Since La spectra possess $g$ values which depart more or less from Landé, the observed Zeeman effects in the table of classified lines are compared with those computed from observed rather than theoretical $g$ 's. This procedure shows in

\footnotetext{
15 W. F. Meggers, J. Wash. Acad. Sci., vol. 17, p. 35, 1927.

${ }_{16} \mathrm{~W}$. F. Meggers, B. S. Jour. Research, vol. 9 (RP468), p. 239,1932

17 R. C. Gibbs and H. E. White, Proc. Nat. Acad. Sci., vol. 12, p. 557, 1926; Phys. Rev., vol. 33, p. 157 1929.

${ }_{18}$ J. S. Badami, Proc. Roy. Soc. London, vol. 43, p. 53, 1931.
} 
the first table, the deviations of observed from theoretical $g$ values and in the second table how closely the observed data are represented by the empirical $g$ values. The observed or computed Zeeman patterns may be compared with the theoretical by reference to "Tables of Theoretical Zeeman Effects" published by Kiess and Meggers. ${ }^{19}$ These remarks apply to the La II and La I spectra (vide infra) as well as to La III.

TABLE 1.-Terms in the La III spectrum

\begin{tabular}{|c|c|c|c|c|c|}
\hline \multirow{2}{*}{$\begin{array}{l}\text { Electron } \\
\text { configura- } \\
\text { tion }\end{array}$} & \multirow[b]{2}{*}{ Terms } & \multirow[b]{2}{*}{ Levels } & \multirow[b]{2}{*}{$\begin{array}{l}\text { Separa- } \\
\text { tions }\end{array}$} & \multicolumn{2}{|c|}{$g$} \\
\hline & & & & $\begin{array}{c}\text { Ob- } \\
\text { serv- } \\
\text { ed }\end{array}$ & Landé \\
\hline $\begin{array}{l}5 d \\
6 s \\
6 p \\
6 d \\
7 s\end{array}$ & 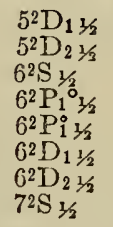 & $\begin{array}{r}0.00 \\
1,603.23 \\
13,590.76 \\
42,014.92 \\
45,110.64 \\
82,378.75 \\
82,812.51 \\
82,345.0\end{array}$ & $\begin{array}{r}3,095.72 \\
433.76\end{array}$ & $\begin{array}{r}2.10 \\
.63 \\
1.37\end{array}$ & $\begin{array}{r}2.000 \\
.667 \\
1.333\end{array}$ \\
\hline
\end{tabular}

It will be shown later that the terms arising from the $4 f$ electron are higher than those from $6 p$ in La I, but lower in La II. This suggested that the La III combinations $5 d\left({ }^{2} \mathrm{D}\right)-4 f\left({ }^{2} \mathrm{~F}\right)$ might lie in the infra-red. The La spark spectrum in the interval 8,000 to 10,500 A was recently explored with xenocyanine plates, but no La III lines were found although La II lines were recorded all the way to $9,893.8 \mathrm{~A}$.

TABLE 2.-Classified lines in the La III spectrum

\begin{tabular}{|c|c|c|c|c|c|}
\hline \multirow{2}{*}{$\begin{array}{l}\lambda \text { (air) } \\
\text { I. A. }\end{array}$} & \multirow{2}{*}{$\begin{array}{l}\text { Inten- } \\
\text { sity }\end{array}$} & \multirow{2}{*}{$\begin{array}{l}\nu(\mathrm{vac}) \\
\mathrm{cm}^{1}\end{array}$} & \multirow{2}{*}{$\begin{array}{l}\text { Term combina- } \\
\text { tions }\end{array}$} & \multicolumn{2}{|c|}{ Zeeman effect } \\
\hline & & & & Observed & Computed \\
\hline $\begin{array}{l}3,517.14 \\
3,171.68 \\
2,684.90 \\
2,682.46 \\
2,651.60\end{array}$ & $\begin{array}{r}200 \\
300 \\
50 \\
30 \\
300\end{array}$ & $\begin{array}{l}28,424.09 \\
31,519.94 \\
37,234.29 \\
37,268.16 \\
37,701.87\end{array}$ & 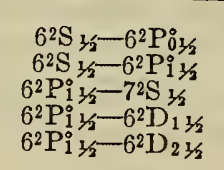 & $\begin{array}{l}(0.73) 1.36 \\
(0.37) 0.99,1.76\end{array}$ & $\begin{array}{l}(0.74) 1.36 \\
(0.36) 1.00,1.74\end{array}$ \\
\hline $\begin{array}{l}2,478.81 \\
2,47.72 \\
2,379.38 \\
2,297.75 \\
2,216.08\end{array}$ & $\begin{array}{r}20 \\
100 \\
200 \\
200 \\
50\end{array}$ & $\begin{array}{l}40,329.9 \\
40,363.79 \\
42,014.92 \\
43,507.40 \\
45,110.63\end{array}$ & $\begin{array}{c}6^{2} \mathrm{P}_{013-72}^{0}-7 \mathrm{~S}_{1 / 2} \\
6^{2} \mathrm{P}_{01 / 2}^{0}-6^{2} \mathrm{D}_{11 / 2} \\
5^{2} \mathrm{D}_{11 / 2}-6^{2} \mathrm{P}_{01 / 2}^{0} \\
5^{2} \mathrm{D}_{21 / 2}-6^{2} \mathrm{P}_{11 / 2}^{0} \\
5^{2} \mathrm{D}_{11 / 2}-6^{2} \mathrm{P}_{11 / 2}^{0}\end{array}$ & & \\
\hline
\end{tabular}

\section{THE SPECTRUM OF SINGLY IONIZED LANTHANUM (La II)}

The spark spectrum of lanthanum is exceptionally complex; more than 800 lines appearing in spark spectrograms are associated with $\mathrm{La}^{+}$atoms. Superficially, the lanthanum spark spectrum resembles the yttrium spark spectrum; in each case the lines are divided roughly into two classes by comparison of arc and spark spectrograms. One

${ }^{19}$ C. C. Kiess and W. F. Meggers, B. S. Jour. Research, vol. 1 (RP23), p. 64, 1928. 
group, including most of the stronger lines, has nearly the same appearance in a 6-ampere, 220 -volt arc and in a high voltage condensed discharge, while the second, lying mainly in the ultra-violet, is greatly enhanced upon passing from the arc to the spark and consists largely of hazy and unsymmetrical lines. The former arise from combinations of low energy states with the next higher or middle set, while the latter are practically all identified as combinations of middle terms with a still higher third set.

The lowest energy states which can arise from $s$ and $d$ type electrons are identified with (even) spectral terms as follows:

\begin{tabular}{|c|c|}
\hline $\begin{array}{c}\text { Electron configuration } \\
s^{2}\end{array}$ & ${ }_{1 \mathrm{~S}}$ Spectral terms \\
\hline$s d$ & $1 \mathrm{D}$ \\
\hline$d^{2}$ & ${ }^{1} \mathrm{~S},{ }^{1} \mathrm{D},{ }^{1} \mathrm{G}$ \\
\hline
\end{tabular}

All of these have been identified except $\left(d^{2}\right)^{1} \mathrm{~S}$. Which particular term will be the lowest energy and represent the normal state of the atom depends on the relative strength of binding of the individual electrons. It is very remarkable that the homologous atoms, $\mathrm{Sc}^{+}$, $\mathrm{Y}^{+}$, and $\mathrm{La}^{+}$, each make a different choice; the normal state of $\mathrm{Sc}^{+}$ is $(s d)^{3} \mathrm{D}$, of $\mathrm{Y}^{+}\left(s^{2}\right)^{1} \mathrm{~S}$, and of $\mathrm{La}^{+}\left(d^{2}\right)^{3} \mathrm{~F}$.

Substitution of a $p$ electron for an $s$ or a $d$ electron produces the following set of (odd) middle terms:

\begin{tabular}{cl} 
Electron configuration & \multicolumn{1}{c}{ Spectral terms } \\
$s p$ & ${ }^{1} \mathrm{P} \quad{ }^{3} \mathrm{P}$ \\
$d p$ & ${ }^{1} \mathrm{P}, 1 \mathrm{D}, 1 \mathrm{~F} \quad{ }^{3} \mathrm{P},{ }^{3} \mathrm{D},{ }^{3} \mathrm{~F}$
\end{tabular}

All of the easily excited lines of $\mathrm{Sc}^{+}$and $\mathrm{Y}^{+}$are accounted for by the above-mentioned low and middle spectral terms, but in the case of $\mathrm{La}^{+}$a large number of otherwise superfluous lines indicate additional middle-set terms to account for which it is necessary to conclude that $f$-type electrons are present. Thus, the substitution of $f$-for $p$-type electrons would yield the following additional (odd) middle-set terms:

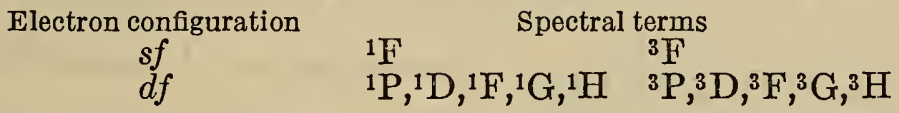

All of these terms have been found in the La II spectrum, they increase the number of middle-set levels from 16 to 40 and thus account for the greater complexity of the spectrum.

The terms produced by the $4 f$ electrons lie lower than those arising from the $6 p$. This is obviously related to the fact that $4 f$ electrons are bound into the normal state of the directly following elements, $\mathrm{Ce}$ to $\mathrm{Lu}$, while the $6 p$ electrons begin to be similarly bound only in Tl. In $\mathrm{Ba}$ I the $f$ electron is much more loosely bound than the $p$. There are numerous high even terms in La II. Those arising from the configurations $5 d 7 s, 5 d 6 d, 6 s 6 d$, and $6 p^{2}$ are homologous with similar terms in Sc II and Y II. Two important additional groups evidently arise from $6 p 4 f$ and $4 f^{2}$. The former are the lowest of all the high even terms. Some hazy lines confined to the spark appear to be combinations between these and still higher odd levels which have been denoted by numbers $1^{\circ}$ to $8^{\circ}$. There are several configurations (for example, $5 d 7 p, 5 d 5 f, 6 p 6 d, 4 f 6 d$ ) which may give rise to levels of this sort, and they must be very numerous. They should combine with the ground terms to give lines in the 
Schumann region. Observations in this region may detect many more such levels and thus lead to the interpretation of the remaining unclassified lines in the visible and near ultra-violet.

This is the most completely developed example of a 2-electron spectrum which has yet been investigated. All the configuration types, $s^{2}, s p, s d, s f, p^{2}, p d, p f, d^{2}, d f, f^{2}$, have been identified and almost all of the terms arising from each. The theoretical relations of the terms arising from each configuration have been discussed by Condon and Shortley. ${ }^{20}$ The agreement of the observed and computed levels is, in general, good, and their theoretical predictions led to the correct identification of the difficult terms $(d f)^{1} \mathrm{H}$ and $\left(f^{2}\right)^{1} \mathrm{I}$.

The terms which have been identified in the La II spectrum are listed in Table 3 , in which term symbols, relative values of the levels, level separations, adopted and Landé $g$ values, and combining terms are given in successive columns. The adopted $g$ values are derived from the observed Zeeman effects for La II lines starting with the completely resolved patterns and then applying the formulas of Shenstone and Blair ${ }^{21}$ to the unresolved blends. For patterns which though unresolved had perpendicular $(n)$ components distinctly shaded outwards $\left(\mathrm{A}^{1}\right)$ or inwards $\left(\mathrm{A}^{2}\right)$ the attempt was made to measure the points of maximum intensity corresponding to the strongest components. If $x$ is the observed separation we should then have

$$
x=J_{1} g_{1}-J_{2} g_{2} \quad\left(J_{1}=J_{2}+1\right)
$$

When no such asymmetry was noticed it was assumed that the settings were on the centroid of the whole pattern and the formula then used was

When $J_{1}=J_{2}$ the equations are

$$
2 x=\left(J_{1}+1\right) g_{1}-J_{2} g_{2}
$$

$$
2 x=g_{1}+g_{2}(n \text { components })
$$

$$
4 / 3 y=(J+1 / 2)(1-X)\left(g_{1}-g_{2}\right) \quad(p \text { components })
$$

where $X=\frac{1}{(2 J+1)^{2}}$ or $\frac{1}{4 J(J+1)}$ according as $J$ is integral or half integral. ${ }^{22}$

The weights assigned to the adopted $g$ 's in Table 3 depend on the number and consistency of the derived values. The probable error corresponding to unit weight is \pm 0.020 .

Most of the g's have nearly the theoretical (Landé) values but marked discrepancies frequently appear which may be attributed to deviations of the actual coupling of the vectors from the ideal SL coupling for narrow multiplets. Some of the largest discordances are clearly due to " $g$ sharing" among neighboring levals with the same $J$; for example, $e^{3} G_{4}, e^{3} F_{4} ; y^{3} D_{1}, y^{3} P_{1}, z^{1} P_{1}$. In these cases the intensities of many combinations are also abnormal.

20 E. U. Condon and C. H. Shortley, Phys. Rev., vol. 37, p. 1025, 1931.

${ }_{21}$ A. G. Shenstone and H. A. Blair, Phil. Mag., vol. 8, p. 765, 1929.

22 H. N. Russell, Phys. Rev., vol. 36, p. 1590, 1930. 
TABLE 3.-Relative terms in the La II spectrum

\begin{tabular}{|c|c|c|c|c|c|c|c|}
\hline \multirow{3}{*}{$\begin{array}{c}\begin{array}{c}\text { Elec- } \\
\text { tron } \\
\text { config- } \\
\text { uration }\end{array} \\
5 d^{2}\end{array}$} & \multirow{3}{*}{ Term } & \multirow{3}{*}{$\begin{array}{l}\text { Level } \\
\begin{array}{r} \\
\\
1,016.10 \\
1,970.70\end{array}\end{array}$} & \multirow{3}{*}{$\begin{array}{r}\begin{array}{c}\text { Level sep- } \\
\text { arations }\end{array} \\
1,016.10 \\
954.60\end{array}$} & \multicolumn{3}{|c|}{$g$} & \multirow{2}{*}{ Combinations } \\
\hline & & & & \multicolumn{2}{|c|}{$\begin{array}{l}\text { Adopted } \\
\text { weight }\end{array}$} & \multirow{2}{*}{$\begin{array}{l}\text { Landé } \\
0.667 \\
1.083 \\
1.250\end{array}$} & \\
\hline & & & & $\begin{array}{l}0.730 \\
1.092 \\
1.258\end{array}$ & $\begin{array}{l}71 / 2 \\
9 \\
8\end{array}$ & & $\left\{\begin{array}{c}z^{3} \mathrm{~F}^{\circ}, z^{1} \mathrm{~F}^{\circ}, z^{1} \mathrm{G}^{\circ}, y^{3} \mathrm{~F}^{\circ}, z^{3} \mathrm{H}^{\circ}, z^{1} \mathrm{D}^{\circ}, z^{3} \mathrm{G}^{\circ}, z^{3} \mathrm{D}^{\circ}, \\
z^{3} \mathrm{P}^{\circ}, y^{1} \mathrm{D}^{\circ}, y^{1} \mathrm{~F}^{\circ}, y^{3} \mathrm{D}^{\circ}, x^{3} \mathrm{~F}^{\circ}, z^{1} \mathrm{P}^{\circ}, y^{3} \mathrm{P}^{\circ}, \\
x^{3} \mathrm{P}^{\circ}, x^{1} \mathrm{~F}^{\circ}, x^{1} \mathrm{P}^{\circ}\end{array}\right.$ \\
\hline $6 s 5 d$ & $a^{1} \mathrm{D}_{2}$ & $1,394.46$ & & .987 & $101 / 2$ & 1.000 & $y^{1} \mathrm{~F}^{\circ}, y^{3} \mathrm{D}^{\circ}, x^{3} \mathrm{~F}^{\circ}, z^{1} \mathrm{P}^{\circ}, y^{3} \mathrm{P}^{\circ}, y^{1} \mathrm{P}^{\circ}, x^{3} \mathrm{P}^{\circ}$, \\
\hline $6 s 5 d$ & $\begin{array}{l}a^{3} D_{1} \\
a^{3} D_{2} \\
a^{3} D_{3}\end{array}$ & $\begin{array}{l}1,895.15 \\
2,591.60 \\
3,250.35\end{array}$ & $\begin{array}{l}696.45 \\
658.75\end{array}$ & $\begin{array}{r}.520 \\
1.140 \\
1.337\end{array}$ & $\begin{array}{r}13 \\
8 \\
11\end{array}$ & $\begin{array}{r}.500 \\
1.167 \\
1.333\end{array}$ & $\left\{\begin{array}{l}{ }^{23} \mathrm{~F}^{\circ}, z^{3} \mathrm{G}^{\circ}, y^{3} \mathrm{~F}^{\circ}, z^{3} \mathrm{H}^{\circ}, z^{1} \mathrm{D}^{\circ}, z^{3} \mathrm{G}^{\circ}, z^{3} \mathrm{D}^{\circ}, z^{3} \mathrm{P}^{\circ}, \\
y^{1} \mathrm{D}^{\circ}, y^{1} \mathrm{~F}^{\circ}, y^{3} \mathrm{D}^{\circ}, x^{3} \mathrm{~F}^{\circ}, z^{1} \mathrm{P}^{\circ}, y^{3} \mathrm{P}^{\circ}, y^{1} \mathrm{P}^{\circ}, \\
x^{3} \mathrm{P}^{\circ}, x^{1} \mathrm{~F}^{\circ}, x^{1} \mathrm{P}^{\circ} .\end{array}\right.$ \\
\hline $5 d^{2}$ & $\begin{array}{l}a^{3} \mathrm{P}_{0} \\
a^{3} \mathrm{P}_{1} \\
a^{3} \mathrm{P}_{2}\end{array}$ & $\begin{array}{l}5,249.70 \\
5,718.12 \\
6,227.42\end{array}$ & $\begin{array}{l}468.42 \\
509.30\end{array}$ & $\begin{array}{r}0 / 0 \\
1.506 \\
1.488\end{array}$ & $\begin{array}{c}6 \\
9 \\
111 / 2\end{array}$ & $\begin{array}{r}0 / 0 \\
1.500 \\
1.500\end{array}$ & ${ }^{3} \mathrm{~F}^{\circ}, z^{1} \mathrm{D}^{\circ}, y^{3} \mathrm{P}^{\circ}, y^{1} \mathrm{P}^{\circ}, z^{\circ}, x^{3} \mathrm{P}^{\circ}, y^{\circ}, x^{1} \mathrm{~F}^{\circ}, y^{\circ}, x^{1} \mathrm{P}^{\circ}, y^{3} \mathrm{D}^{\circ}, x^{3} \mathrm{~F}^{\circ}$, \\
\hline $\begin{array}{l}6 s^{2} \\
5 d^{2}\end{array}$ & $\begin{array}{l}a^{2} \\
a^{1} \mathrm{~S}_{0}\end{array}$ & $\begin{array}{l}7,394.57 \\
7,473.32\end{array}$ & & $\begin{array}{c}0 / 0 \\
1.003\end{array}$ & $\begin{array}{c}4 \\
2\end{array}$ & $\begin{array}{r}0 / 0 \\
1.000\end{array}$ & $\begin{array}{l}z^{3} \mathrm{D}^{\circ}, z^{3} \mathrm{P}^{\circ}, y^{3} \mathrm{D}^{\circ}, z^{1} \mathrm{P}^{\circ}, y^{1} \mathrm{P}^{\circ}, x^{3} \mathrm{P}^{\circ}, x^{1} \mathrm{P}^{\circ} \\
z^{1} \mathrm{G}^{\circ}, y^{3} \mathrm{~F}^{\circ}, z^{3} \mathrm{H}^{\circ}, z^{3} \mathrm{G}^{\circ}, z^{3} \mathrm{D}^{\circ}, y^{1} \mathrm{~F}^{\circ}, y^{3} \mathrm{D}^{\circ},\end{array}$ \\
\hline $5 d^{2}$ & $b^{1} \mathrm{D}_{2}$ & $10,094.86$ & & 1. 015 & 70 & 1.000 & $z^{3} \mathrm{D}^{\circ}, z^{\circ}, x^{3} \mathrm{P}^{\circ}, y^{1} \mathrm{D}^{\circ} \mathrm{P}^{\circ} y^{3} \mathrm{D}^{\circ}, x^{3} \mathrm{~F}^{\circ}, z^{1} \mathrm{P}^{\circ}, y^{3} \mathrm{P}^{\circ}, y^{1} \mathrm{P}^{\circ}$, \\
\hline $6 s 4 f$ & $\begin{array}{l}z^{3} \mathrm{~F}_{2}^{\circ} \\
2^{3} \mathrm{~F}_{3}^{\circ} \\
z^{3} \mathrm{~F}_{4}^{\circ}\end{array}$ & $\begin{array}{l}14,147.98 \\
14,375.17 \\
15,698.74\end{array}$ & $\begin{array}{l}227.19 \\
323.57\end{array}$ & $\begin{array}{l}.676 \\
1.087 \\
1.258\end{array}$ & $\begin{array}{r}7 \\
10 \\
9\end{array}$ & $\begin{array}{l}.667 \\
1.083 \\
1.250\end{array}$ & $\left\{\begin{array}{l}a^{3} \mathrm{~F}, a^{1} \mathrm{D}, a^{3} \mathrm{D}, e^{3} \mathrm{G}, e^{3} \mathrm{~F}, e^{1} \mathrm{~F}, e^{3} \mathrm{D}, e^{1} \mathrm{G}, f^{3} \mathrm{D}, \\
f^{1} \mathrm{~F}, f^{3} \mathrm{G}, f^{3} \mathrm{~F}, g^{3} \mathrm{D}, e^{3} \mathrm{H}, g^{3} \mathrm{~F}, h^{1} \mathrm{D} .\end{array}\right.$ \\
\hline $6 s 4 f$ & $z^{1} \mathrm{~F}_{3}^{\circ}$ & $15,773.77$ & & 1. 047 & 10 & 1.000 & $a^{3} \mathrm{~F}, a^{1} \mathrm{D}, e^{3} \mathrm{G}, e^{3} \mathrm{~F}, e^{1} \mathrm{~F}, e^{3} \mathrm{D}, e^{1} \mathrm{G}, e^{1} \mathrm{D}, f^{1} \mathrm{D}$, \\
\hline $5 d 4 f$ & $z^{1} G_{i}^{0}$ & $16,599.17$ & & .996 & 4 & 1.000 & $\begin{array}{l}a^{3} \mathrm{~F}, a^{3} \mathrm{D}, a^{1} \mathrm{G}, e^{3} \mathrm{G}, e^{3} \mathrm{~F}, e^{1} \mathrm{~F}, e^{1} \mathrm{G}, f^{1} \mathrm{~F}, f^{3} \mathrm{G}, f^{1} \mathrm{G}, \\
e^{3} \mathrm{H}, g^{3} \mathrm{~F}, g^{1} \mathrm{G} .\end{array}$ \\
\hline $5 d 4 f$ & $\begin{array}{l}y^{3} \mathrm{~F}_{2}^{\circ} \\
y^{3} \mathrm{~F}_{3}^{\circ} \\
y^{3} \mathrm{~F}_{4}^{\circ}\end{array}$ & $\begin{array}{l}17,211.93 \\
18,235.56 \\
19,214.54\end{array}$ & $\begin{array}{r}1,023.63 \\
978.98\end{array}$ & $\begin{array}{l}.757 \\
1.090 \\
1.243\end{array}$ & $\begin{array}{l}8 \\
8 \\
6\end{array}$ & $\begin{array}{l}.667 \\
1.083 \\
1.250\end{array}$ & $\left\{\begin{array}{l}a^{3} \mathrm{~F}, a^{1} \mathrm{D}, a^{3} \mathrm{D}, a^{3} \mathrm{P}, a^{1} \mathrm{G}, e^{3} \mathrm{G}, e^{3} \mathrm{~F}, e^{3} \mathrm{D}, e^{1} \mathrm{G}, \\
e^{1} \mathrm{D}, f^{1} \mathrm{D}, f^{1} \mathrm{~F}, f^{3} \mathrm{G}, g^{3} \mathrm{D}, f^{1} \mathrm{G}, g^{3} \mathrm{~F}, g^{1} \mathrm{G}, h^{1} \mathrm{D}, \\
f^{3} \mathrm{P}, i^{1} \mathrm{D} .\end{array}\right.$ \\
\hline $5 d 4 f$ & $\begin{array}{l}z^{3} \mathrm{H}_{4}^{\circ} \\
z^{3} \mathrm{H}_{5}^{\circ} \\
z^{3} \mathrm{H}_{6}^{\circ}\end{array}$ & $\begin{array}{l}17,825.62 \\
18,580.41 \\
19,749.62\end{array}$ & $\begin{array}{r}754.79 \\
1,169.21\end{array}$ & $\begin{array}{l}.865 \\
1.082 \\
1.17\end{array}$ & $\begin{array}{l}2 \\
4 \\
1\end{array}$ & $\begin{array}{l}.800 \\
1.033 \\
1.167\end{array}$ & $\left\{\begin{array}{l}a^{3} \mathrm{~F}, a^{3} \mathrm{D}, a^{1} \mathrm{G}, e^{3} \mathrm{G}, e^{3} \mathrm{~F}, e^{1} \mathrm{~F}, e^{1} \mathrm{G}, f^{3} \mathrm{~F}, f^{1} \mathrm{G}, e^{3} \mathrm{H} \\
g^{3} \mathrm{~F}, g^{1} \mathrm{G}, e^{1} \mathrm{I} .\end{array}\right.$ \\
\hline $5 d 4 f$ & $z^{1} \mathrm{D}_{2}^{\circ}$ & $18,895.41$ & & .931 & $31 / 2$ & 1.000 & $\begin{array}{l}a^{3} \mathrm{~F}, a^{1} \mathrm{D}, a^{3} \mathrm{D}, a^{3} \mathrm{P}, e^{3} \mathrm{G}, e^{3} \mathrm{~F}, e^{1} \mathrm{~F}, e^{3} \mathrm{D}, e^{1} \mathrm{D}, f^{3} \mathrm{D}, \\
f^{1} \mathrm{~F}, f^{3} \mathrm{G}, f^{3} \mathrm{~F}, g^{3} \mathrm{D}, e^{1} \mathrm{P}, e^{3} \mathrm{~S}, g^{3} \mathrm{~F}, h^{1} \mathrm{D}, g^{3} \mathrm{P}, h^{3} \mathrm{D}, \\
f^{3} \mathrm{P}, i^{1} \mathrm{D} .\end{array}$ \\
\hline $5 d 4 f$ & $\begin{array}{l}z^{3} \mathrm{G}_{3}^{\circ} \\
z^{3} \mathrm{G}_{4}^{\circ} \\
z^{3} \mathrm{G}_{5}^{\circ}\end{array}$ & $\begin{array}{l}20,402.82 \\
21,331.60 \\
22,282.90\end{array}$ & $\begin{array}{l}928.78 \\
951.30\end{array}$ & $\begin{array}{l}.775 \\
1.060 \\
1.203\end{array}$ & $\begin{array}{l}4 \\
6 \\
5\end{array}$ & $\begin{array}{l}.750 \\
1.050 \\
1.200\end{array}$ & $\left\{\begin{array}{l}a^{3} \mathrm{~F}, a^{1} \mathrm{D}, a^{3} \mathrm{D}, a^{1} \mathrm{G}, e^{3} \mathrm{G}, e^{3} \mathrm{~F}, e^{1} \mathrm{~F}, e^{3} \mathrm{D}, e^{1} \mathrm{D}, f^{3} \mathrm{G} \\
f^{3} \mathrm{~F}, g^{1} \mathrm{D}, f^{1} \mathrm{G}, e^{3} \mathrm{H}, g^{3} \mathrm{~F}, g^{1} \mathrm{G} .\end{array}\right.$ \\
\hline $5 d 4 f$ & $\begin{array}{l}z^{3} \mathrm{D}_{1}^{\circ} \\
z^{3} \mathrm{D}_{2}^{\circ} \\
z^{3} \mathrm{D}_{3}^{\circ}\end{array}$ & $\begin{array}{l}21,441.73 \\
22,106.02 \\
22,537.30\end{array}$ & $\begin{array}{l}664.29 \\
431.28\end{array}$ & $\begin{array}{l}.547 \\
1.186 \\
1.317\end{array}$ & $\begin{array}{r}8 \\
12 \\
10\end{array}$ & $\begin{array}{l}.500 \\
1.167 \\
1.333\end{array}$ & $\left\{\begin{array}{l}a^{3} \mathrm{~F}, a^{1} \mathrm{D}, a^{3} \mathrm{D}, a^{3} \mathrm{P}, a^{1} \mathrm{~S}, a^{1} \mathrm{G}, b^{1} \mathrm{D}, e^{3} \mathrm{G}, e^{3} \mathrm{~F}, e^{1 \mathrm{~F}}, \\
e^{3} \mathrm{D}, e^{1} \mathrm{G}, e^{1} \mathrm{D}, f^{3} \mathrm{D}, f^{1} \mathrm{D}, f^{3} \mathrm{G}, f^{3} \mathrm{~F}, g^{3} \mathrm{D}, e^{1 \mathrm{P}}, e^{3} \mathrm{~S}, \\
e^{3} \mathrm{P}, g^{1} \mathrm{D}, f^{1} \mathrm{G}, e^{3} \mathrm{H}, g^{3} \mathrm{~F}, h^{1} \mathrm{D}, g^{3} \mathrm{P}, f^{3} \mathrm{P}, i^{1} \mathrm{D} .\end{array}\right.$ \\
\hline $5 d 4 f$ & $\begin{array}{l}z^{3} \mathrm{P}_{0}^{0} \\
z^{3} \mathrm{P}_{1}^{\circ} \\
z^{3} \mathrm{P}_{2}^{0}\end{array}$ & $\begin{array}{l}22,68 \\
22,70 \\
23,24\end{array}$ & $\begin{array}{r}21.45 \\
541.78\end{array}$ & $\begin{array}{r}0 / 0 \\
1.458 \\
1.462\end{array}$ & $\begin{array}{l}7 \\
7\end{array}$ & $\begin{array}{r}0 / 0 \\
1.500 \\
1.500\end{array}$ & $\left\{\begin{array}{l}a^{3} \mathrm{~F}, a^{1} \mathrm{D}, a^{3} \mathrm{D}, a^{3} \mathrm{P}, a^{1 \mathrm{~S}}, b^{1} \mathrm{D}, e^{3} \mathrm{D}, e^{1 \mathrm{D}}, j^{3} \mathrm{D}, f^{1} \mathrm{~F}, \\
g^{3} \mathrm{D}, e^{1} \mathrm{P}, e^{2} \mathrm{~S}, e^{3} \mathrm{P}, g^{1} \mathrm{D}, g^{3} \mathrm{~F}, h^{1} \mathrm{D}, g^{3} \mathrm{P}, h^{3} \mathrm{D}, f^{3} \mathrm{P} \text {, } \\
i^{1} \mathrm{D} .\end{array}\right.$ \\
\hline $6 p 5 d$ & $y^{1} \mathrm{D}_{2}^{\circ}$ & $24,462.66$ & & .897 & 71 & 1.000 & $\begin{array}{l}a^{3} \mathrm{~F}, a^{1} \mathrm{D}, a^{3} \mathrm{D}, a^{3} \mathrm{P}, b^{1} \mathrm{D}, e^{3} \mathrm{G}, e^{3} \mathrm{D}, f^{3} \mathrm{D}, f^{1} \mathrm{D}, f^{1} \mathrm{~F}, \\
f^{3} \mathrm{G}, f^{3} \mathrm{~F}, y^{3} \mathrm{D}, e^{1} \mathrm{P}, e^{3} \mathrm{P}, h^{1} \mathrm{D}, f^{3} \mathrm{P}, i^{1} \mathrm{D} .\end{array}$ \\
\hline $5 d 4 f$ & $y^{1} \mathrm{~F}_{3}^{\circ}$ & $24,522.70$ & & 1.032 & 8 & 1.000 & $\begin{array}{l}a^{3} \mathrm{~F}, a^{1} \mathrm{D}, a^{3} \mathrm{D}, a^{3} \mathrm{P}, a^{1} \mathrm{G}, e^{3} \mathrm{G}, e^{1} \mathrm{~F}, e^{3} \mathrm{D}, e^{1} \mathrm{G}, e^{1} \mathrm{D}, \\
f^{1} \mathrm{~F}, g^{3} \mathrm{D}, g^{1} \mathrm{G}, h^{1} \mathrm{D}, g^{3} \mathrm{P}, f^{3} \mathrm{P}, i^{1} \mathrm{D} .\end{array}$ \\
\hline $6 p 5 d$ & $\begin{array}{l}y^{3} \mathrm{D}_{1}^{\circ} \\
y^{3} \mathrm{D}_{2}^{\circ} \\
y^{3} \mathrm{D}_{3}^{\circ}\end{array}$ & $\begin{array}{l}25,973.37 \\
27,388.11 \\
28,315.25\end{array}$ & $\begin{array}{r}1,414.74 \\
927.14\end{array}$ & $\begin{array}{r}.799 \\
1.191 \\
1.312\end{array}$ & \begin{tabular}{r|r}
8 \\
10 \\
7
\end{tabular} & $\begin{array}{l}.500 \\
1.167 \\
1.333\end{array}$ & $\left\{\begin{array}{l}a^{3} \mathrm{~F}, a^{1} \mathrm{D}, a^{3} \mathrm{D}, a^{3} \mathrm{P}, a^{1} \mathrm{~S}, a^{1 \mathrm{G}}, b^{1} \mathrm{D}, e^{3} \mathrm{~F}, e^{3} \mathrm{D}, e^{1} \mathrm{D} \\
f^{3} \mathrm{D}, f^{3} \mathrm{G}, f^{3} \mathrm{~F}, g^{3} \mathrm{D}, e^{1 \mathrm{P}}, e^{3} \mathrm{~S}, e^{3} \mathrm{P}, g^{1} \mathrm{D}, f^{1} \mathrm{G}, e^{3} \mathrm{H}, \\
h^{1} \mathrm{D}, g^{3} \mathrm{P}, h^{3} \mathrm{D}, f^{3} \mathrm{P}, i^{3} \mathrm{D} .\end{array}\right.$ \\
\hline $6 p 5 d$ & $\begin{array}{l}x^{3} \mathrm{~F}_{2}^{\circ} \\
x^{3} \mathrm{~F}_{3}^{\circ} \\
x^{3} \mathrm{~F}_{4}^{\circ}\end{array}$ & $\begin{array}{l}26,414.01 \\
26,837.66 \\
28,565.40\end{array}$ & $\begin{array}{r}423.65 \\
1,727.74\end{array}$ & $\begin{array}{l}.836 \\
1.081 \\
1.246\end{array}$ & $\begin{array}{l}71 / 2 \\
6 \\
4\end{array}$ & $\begin{array}{l}.667 \\
1.083 \\
1.250\end{array}$ & $\left\{\begin{array}{l}a^{3} \mathrm{~F}, a^{1} \mathrm{D}, a^{3} \mathrm{D}, a^{3} \mathrm{P}, a^{1} \mathrm{G}, b^{1} \mathrm{D}, e^{3} \mathrm{G}, e^{3} \mathrm{~F}, e^{1} \mathrm{~F}, e^{3} \mathrm{D} \\
e^{1} \mathrm{D}, f^{3} \mathrm{D}, f^{1} \mathrm{D}, f^{1} \mathrm{~F}, f^{3} \mathrm{G}, f^{3} \mathrm{~F}, g^{3} \mathrm{D}, e^{1} \mathrm{P}, e^{3} \mathrm{~S}, g^{1} \mathrm{D}, \\
f^{1} \mathrm{G}, e^{3} \mathrm{H}, h^{1} \mathrm{D}, g^{3} \mathrm{P}, i^{1} \mathrm{D}, i^{3} \mathrm{D} .\end{array}\right.$ \\
\hline $5 d 4 f$ & $2^{1} \mathrm{P}_{1}^{4}$ & $27,423.91$ & & .882 & 9 & 1. 000 & 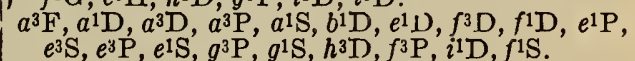 \\
\hline $\begin{array}{l}5 d 4 f \\
6 p 5 d\end{array}$ & $\begin{array}{l}21 \mathrm{H}_{5}^{:} \\
y^{3} \mathrm{P}_{0}^{0}\end{array}$ & $\begin{array}{l}28,525.71 \\
27,545.85\end{array}$ & & $\begin{array}{c}1.00 \\
0 / 0\end{array}$ & 1 & $\begin{array}{r}1.000 \\
0 / 0\end{array}$ & $a^{1} \mathrm{G}, e^{1} \mathrm{G}, e^{3} \mathrm{H}, g^{1} \mathrm{G}, e^{1} \mathrm{I}$ \\
\hline & $\begin{array}{l}y^{3} \mathrm{P}^{0} \mathrm{P}_{1}^{0} \\
y^{3} \mathrm{P}_{2}^{0}\end{array}$ & $\begin{array}{l}0.85 \\
4.55 \\
18.05\end{array}$ & $\begin{array}{r}608.70 \\
1,343.50\end{array}$ & $\begin{array}{l}0 / 0 \\
1.262 \\
1.495\end{array}$ & $\begin{array}{l}5 \\
5\end{array}$ & $\begin{array}{l}0 / 0 \\
1.500 \\
1.500\end{array}$ & $\left\{\begin{array}{l}a^{3} \mathrm{~F}, a^{1} \mathrm{D}, a^{3} \mathrm{D}, a^{3} \mathrm{P}, b^{1} \mathrm{D}, e^{3} \mathrm{D}, f^{3} \mathrm{D}, f^{1} \mathrm{D}, g^{3} \mathrm{D}, e^{3} \mathrm{~S} \\
e^{3} \mathrm{P}, g^{1} \mathrm{D}, g^{3} \mathrm{P}, g^{1} \mathrm{~S}, h^{3} \mathrm{D}, f^{3} \mathrm{P}, i^{1} \mathrm{D}, f^{1} \mathrm{~S} .\end{array}\right.$ \\
\hline $6 p 5 d$ & $y^{1} \mathrm{P}_{1}^{6}$ & $30,353.33$ & & 1. 066 & 6 & 1.000 & $\begin{array}{l}a^{1} \mathrm{D}, a^{3} \mathrm{D}, a^{3} \mathrm{P}, b^{1} \mathrm{D}, e^{1} \mathrm{D}, f^{1} \mathrm{D}, f^{3} \mathrm{~F}, e^{1 \mathrm{P}}, e^{3} \mathrm{P}, e^{1} \mathrm{~S}, \\
g^{1} \mathrm{~S}, h^{3} \mathrm{D}, i^{1} \mathrm{D}, f^{1} \mathrm{~S},{ }^{1} \mathrm{D} ?\end{array}$ \\
\hline $6 s 6 p$ & $\begin{array}{l}x^{3} \mathrm{P}_{0}^{\circ} \\
x^{3} \mathrm{P}_{1}^{0} \\
x^{3} \mathrm{P}_{2}^{0}\end{array}$ & $\begin{array}{l}31,785.82 \\
32,160.99 \\
33,204.41\end{array}$ & $\begin{array}{r}375.17 \\
1,043.42\end{array}$ & $\begin{array}{r}0 / 0 \\
1.515 \\
1.478\end{array}$ & $\begin{array}{l}6 \\
4\end{array}$ & $\begin{array}{r}0 / 0 \\
1.500 \\
1.500\end{array}$ & $\left\{\begin{array}{l}a^{3} \mathrm{~F}, a^{1} \mathrm{D}, a^{3} \mathrm{D}, a^{3} \mathrm{P}, a^{1} \mathrm{~S}, b^{1} \mathrm{D}, f^{3} \mathrm{G}, g^{3} \mathrm{D}, e^{1 \mathrm{P}}, e^{3} \mathrm{~S}, \\
e^{1} \mathrm{~S}, g^{3} \mathrm{P}, h^{3} \mathrm{D}, f^{3} \mathrm{P} .\end{array}\right.$ \\
\hline $6 p 5 d$ & $x^{1} \mathrm{~F}_{3}^{\circ}$ & $32,201.05$ & & 1.006 & 7 & 1.000 & $\begin{array}{c}a^{3} \mathrm{~F}, a^{1} \mathrm{D}, a^{3} \mathrm{D}, a^{3} \mathrm{P}, a^{1} \mathrm{G}, b^{1} \mathrm{D}, f^{3} \mathrm{D}, f^{1} \mathrm{D}, f^{1} \mathrm{~F}, f^{3} \mathrm{G}, \\
f^{1} \mathrm{G}, g^{3} \mathrm{~F}, g^{1} \mathrm{G}, h^{1} \mathrm{D}, i^{1} \mathrm{D},{ }^{1} \mathrm{D} ?\end{array}$ \\
\hline $6 p 4 f$ & $\begin{array}{l}e^{3} G_{3} \\
e^{3} G_{4} \\
e^{3} G_{3}\end{array}$ & $\begin{array}{l}35,452.66 \\
37,172.79 \\
39,018.74\end{array}$ & $\begin{array}{l}1,720.13 \\
1,845.95\end{array}$ & $\begin{array}{l}.873 \\
1.146 \\
1.203\end{array}$ & $\begin{array}{l}5 \\
7 \\
3\end{array}$ & $\begin{array}{l}.750 \\
1.050 \\
1.200\end{array}$ & $\left\{\begin{array}{l}z^{3} \mathrm{~F}^{\circ}, z^{1} \mathrm{~F}^{\circ}, z^{1} \mathrm{G}^{\circ}, y^{3} \mathrm{~F}^{\circ}, z^{3} \mathrm{H}^{\circ}, z^{1} \mathrm{D}^{\circ}, z^{3} \mathrm{G}^{\circ}, z^{3} \mathrm{D}^{\circ}, \\
y^{1} \mathrm{D}^{\circ}, y^{1} \mathrm{~F}^{\circ}, x^{3} \mathrm{~F}^{\circ} .\end{array}\right.$ \\
\hline $6 p 4 f$ & $\begin{array}{l}e^{3} \mathrm{~F}_{2} \\
e^{3} \mathrm{~F}_{3} \\
e^{3} \mathrm{~F}_{4}\end{array}$ & $\begin{array}{l}35,787.53 \\
36,954.65 \\
37,790.57\end{array}$ & $\begin{array}{r}1,167.12 \\
835.92\end{array}$ & $\begin{array}{l}.732 \\
1.061 \\
1.141\end{array}$ & $\begin{array}{l}5 \\
71 / 2 \\
7\end{array}$ & $\begin{array}{l}.667 \\
1.083 \\
1.250\end{array}$ & $\left\{\begin{array}{l}z^{3} \mathrm{~F}^{\circ}, z^{1} \mathrm{~F}^{\circ}, z^{1} \mathrm{G}^{\circ}, y^{3} \mathrm{~F}^{\circ}, z^{3} \mathrm{H}^{\circ}, z^{1} \mathrm{D}^{\circ}, z^{3} \mathrm{G}^{\circ}, z^{3} \mathrm{D}^{\circ}, \\
y^{3} \mathrm{D}^{\circ}, x^{3} \mathrm{~F}^{\circ} .\end{array}\right.$ \\
\hline $6 p 4 f$ & $e^{1} \mathrm{~F}_{3}$ & & & 053 & 6 & 1.000 & $z^{3} \mathrm{~F}^{\circ}, z^{1} \mathrm{~F}^{\circ}, z^{1} \mathrm{G}^{\circ}, z^{3} \mathrm{H}^{\circ}, z^{1} \mathrm{D}^{\circ}, z^{3} \mathrm{G}^{\circ}, z^{3} \mathrm{D}^{\circ}, y^{1} \mathrm{~F}^{\circ}$, \\
\hline $6 p 4 f$ & $e^{1} G_{1}$ & $39,221.65$ & & 1.060 & 6 & 1. 000 & $\begin{array}{l}z^{3} \mathrm{~F}^{\circ}, z^{1} \mathrm{~F}^{\circ}, z^{1} \mathrm{G}^{\circ}, y^{3} \mathrm{~F}^{\circ}, z^{3} \mathrm{H}^{\circ}, z^{3} \mathrm{G}^{\circ}, z^{3} \mathrm{D}^{\circ}, y^{1} \mathrm{~F}^{\circ}, \\
z^{\circ},\end{array}$ \\
\hline
\end{tabular}


TABLE 3.-Relative terms in the La II spectrum-Continued

\begin{tabular}{|c|c|c|c|c|c|c|c|}
\hline \multirow{3}{*}{$\begin{array}{c}\begin{array}{c}\text { Elec- } \\
\text { tron } \\
\text { config- } \\
\text { uration }\end{array} \\
\epsilon p 4 f\end{array}$} & \multirow{3}{*}{$\begin{array}{l}\text { Term } \\
e^{3} \mathrm{D}_{1} \\
e^{3} \mathrm{D}_{2} \\
e^{3} \mathrm{D}_{3}\end{array}$} & \multirow{3}{*}{$\begin{array}{c}\text { Level } \\
38,534.11 \\
38,221.49 \\
39,402.55\end{array}$} & \multirow{4}{*}{$\begin{array}{c}\begin{array}{c}\text { Level sep- } \\
\text { arations }\end{array} \\
-312.62 \\
1,181.06\end{array}$} & \multicolumn{3}{|c|}{$g$} & \multirow{2}{*}{ Combinations } \\
\hline & & & & \multicolumn{2}{|c|}{$\begin{array}{l}\text { Adopted } \\
\text { weight }\end{array}$} & Landé & \\
\hline & & & & $\begin{array}{l}0.495 \\
1.100 \\
1.306\end{array}$ & $\begin{array}{l}6 \\
7 \\
9\end{array}$ & $\begin{array}{l}0.500 \\
1.167 \\
1.333\end{array}$ & $\left\{\begin{array}{l}z^{3} \mathrm{~F}^{\circ}, z^{1} \mathrm{~F}^{\circ}, z^{3} \mathrm{~F}^{\circ}, z^{1} \mathrm{D}^{\circ}, z^{3} \mathrm{G}^{\circ}, z^{3} \mathrm{D}^{\circ}, z^{3} \mathrm{P}^{\circ}, y^{1} \mathrm{D}^{\circ}, \\
x^{3} \mathrm{~F}^{\circ}, y^{3} \mathrm{P}^{\circ}\end{array}\right.$ \\
\hline $6 p 4 f$ & $e^{1} \mathrm{D}_{2}$ & $40,457.71$ & & 1. 046 & 5 & 1. 000 & $\begin{array}{c}z^{1} \mathrm{~F}^{\circ}, y^{3} \mathrm{~F}^{\circ}, z^{1} \mathrm{D}^{\circ}, z^{3} \mathrm{G}^{\circ}, z^{3} \mathrm{D}^{\circ}, z^{3} \mathrm{P}^{\circ}, y^{1} \mathrm{~F}^{\circ}, y^{3} \mathrm{D}^{\circ}, \\
x^{3} \mathrm{~F}^{\circ}, y^{1} \mathrm{P}^{\circ} .\end{array}$ \\
\hline $\begin{array}{l}6 s 6 p \\
5 d 7 s\end{array}$ & $\begin{array}{l}x^{1} \mathrm{P}_{1} \\
f^{3} \mathrm{D}_{1} \\
f^{3} \mathrm{D}_{2} \\
f^{3} \mathrm{D}_{3}\end{array}$ & $\begin{array}{l}45,692.17 \\
49,733.13 \\
49,884.35 \\
51,228.57\end{array}$ & $\begin{array}{r}151.22 \\
1,344.22\end{array}$ & $\begin{array}{l}.97 \\
.520 \\
1.141 \\
1.331\end{array}$ & \begin{tabular}{l|}
1 \\
3 \\
$31 / 2$ \\
3
\end{tabular} & $\begin{array}{l}1.000 \\
.500 \\
1.167 \\
1.333\end{array}$ & $\left\{\begin{array}{l}a^{3 \mathrm{~F}}, a^{1} \mathrm{D}, a^{3} \dot{\mathrm{D}}, a^{3} \mathrm{P}, a^{1} \mathrm{~S}, b^{1} \mathrm{D}, i^{1} \mathrm{D} . \\
z^{3} \mathrm{~F}^{\circ}, z^{1} \mathrm{D}^{\circ}, z^{3} \mathrm{D}^{\circ}, z^{3} \mathrm{P}^{\circ}, y^{1} \mathrm{D}^{\circ}, y^{3} \mathrm{D}^{\circ}, x^{3} \mathrm{~F}^{\circ}, z^{1} \mathrm{P}^{\circ}, \\
{ }^{3} y \mathrm{P}^{\circ}, x^{1} \mathrm{~F}^{\circ} .\end{array}\right.$ \\
\hline $5 d 7 s$ & $f 1 \mathrm{D}_{2}$ & $51,523.86$ & & 1. 02 & $11 / 2$ & 1. 000 & $\underset{x^{1} \mathrm{~F}^{\circ}}{\mathrm{F}^{\circ}}, y^{3} \mathrm{~F}^{\circ}, z^{3} \mathrm{D}^{\circ}, y^{1} \mathrm{D}^{\circ}, x^{3} \mathrm{~F}^{\circ}, z^{1} \mathrm{P}^{\circ}, y^{3} \mathrm{P}^{\circ}, y^{1} \mathrm{P}^{\circ}$, \\
\hline $5 d 6 d$ & $f^{1} \mathrm{~F}_{3}$ & $52,137.67$ & & 1.00 & 1 & 1. 000 & $\begin{array}{c}z^{3} \mathrm{~F}^{\circ}, z^{1} \mathrm{G}^{\circ}, y^{3} \mathrm{~F}^{\circ}, z^{1} \mathrm{D}^{\circ}, z^{3} \mathrm{P}^{\circ}, y^{1} \mathrm{D}^{\circ}, y^{1} \mathrm{~F}^{\circ}, x^{3} \mathrm{~F}^{\circ}, \\
x^{\prime}\end{array}$ \\
\hline $5 d 6 d$ & $\begin{array}{l}f^{3} \mathrm{G}_{3} \\
f^{3} \mathrm{G}_{4} \\
f^{3} \mathrm{G}_{5}\end{array}$ & $\begin{array}{l}52,857.88 \\
53,333.37 \\
54,434.65\end{array}$ & $\begin{array}{r}475.49 \\
1,101.28\end{array}$ & $\begin{array}{l}.89 \\
1.05 \\
1.20\end{array}$ & $\begin{array}{l}1 \\
1 \\
1\end{array}$ & $\begin{array}{r}.750 \\
1.050 \\
1.200\end{array}$ & $z^{3} \mathrm{~F}^{\circ}, z^{1} \mathrm{~F}^{\circ}, z^{1} \mathrm{G}^{\circ}, y^{3} \mathrm{~F}^{\circ}, z^{3} \mathrm{G}^{\circ}, x^{3} \mathrm{~F}^{\circ}, x^{1} \mathrm{~F}^{\circ}$. \\
\hline $5 d 6 d$ & $\begin{array}{l}f^{3} \mathrm{~F}_{2} \\
f^{3} \mathrm{~F}_{3} \\
f^{3} \mathrm{~F}_{4}\end{array}$ & $\begin{array}{l}53,885.24 \\
54,840.04 \\
55,321.35\end{array}$ & $\begin{array}{l}954.80 \\
481.31\end{array}$ & $\begin{array}{l}.77 \\
1.14 \\
1.16\end{array}$ & $\begin{array}{l}2 \\
2 \\
2\end{array}$ & $\begin{array}{l}.667 \\
1.083 \\
1.250\end{array}$ & $\left\{\begin{array}{l}z^{3} \mathrm{~F}^{\circ}, z^{1} \mathrm{~F}^{\circ}, z^{3} \mathrm{H}^{\circ}, z^{1} \mathrm{D}^{\circ}, z^{3} \mathrm{G}^{\circ}, z^{3} \mathrm{D}^{\circ}, y^{1} \mathrm{D}^{\circ}, y^{3} \mathrm{D}^{\circ}, \\
x^{3} \mathrm{~F}^{\circ}\end{array}\right.$ \\
\hline $5 d 6 d$ & $\begin{array}{l}g^{3} \mathrm{D}_{1} \\
g^{3} \mathrm{D}_{2} \\
g^{3} \mathrm{D}_{3}\end{array}$ & $\begin{array}{l}52,169.66 \\
52,734.81 \\
53,689.56\end{array}$ & $\begin{array}{l}565.15 \\
954.75\end{array}$ & $\begin{array}{r}.67 \\
1.17 \\
1.21\end{array}$ & $\begin{array}{l}3 \\
1 \\
3\end{array}$ & $\begin{array}{r}.500 \\
1.167 \\
1.333\end{array}$ & $\left\{\begin{array}{l}z^{1} \mathrm{~F}^{\circ}, y^{3} \mathrm{~F}^{\circ}, z^{1} \mathrm{D}^{\circ}, z^{3} \mathrm{D}^{\circ}, z^{3} \mathrm{P}^{\circ}, y^{1} \mathrm{D}^{\circ}, y^{1} \mathrm{~F}^{\circ}, y^{3} \mathrm{D}^{\circ}, y^{3} \mathrm{P}^{\circ}, x^{3} \mathrm{P}^{\circ} \\
\end{array}\right.$ \\
\hline $5 d 6 d$ & $e^{3} \mathrm{~S}_{1}$ & $54,365.80$ & & 1.42 & 1 & 2.000 & $z^{1} \mathrm{D}^{\circ} \mathrm{P}^{z^{3}} \mathrm{D}^{\circ}, z^{3} \mathrm{P}^{\circ}, y^{3} \mathrm{D}^{\circ}, x^{3} \mathrm{~F}^{\circ}, z^{1} \mathrm{P}^{\circ}, y^{3} \mathrm{P}^{\circ}, y^{1} \mathrm{P}^{\circ}$, \\
\hline $5 d 6 d$ & $e^{1} \mathrm{P}_{1}$ & $53,302.56$ & & & & & $z^{1} \mathrm{D}^{\circ} \mathrm{P}^{\circ} \dot{z}^{3} \mathrm{D}^{\circ}, z^{3} \mathrm{P}^{\circ}, y^{1} \mathrm{D}^{\circ}, y^{3} \mathrm{D}^{\circ}, x^{3} \mathrm{~F}^{\circ}, z^{1} \mathrm{P}^{\circ}, y^{1} \mathrm{P}^{\circ}$, \\
\hline $5 d 6 d$ & $\begin{array}{l}e^{3} \mathrm{P}_{0} \\
e^{3} \mathrm{P}_{1} \\
e^{3} \mathrm{P}_{2}\end{array}$ & $\begin{array}{l}54,964.19 ? \\
55,230.33 \\
56,036.60\end{array}$ & 266. 14 & $\begin{array}{l}1.57 \\
1.22\end{array}$ & $\begin{array}{l}1 \\
1\end{array}$ & $\begin{array}{l}1.500 \\
1.500\end{array}$ & $z^{3} \mathrm{D}^{\circ}, z^{3} \mathrm{P}^{\circ}, y^{1} \mathrm{D}^{\circ}, y^{3} \mathrm{D}^{\circ}, z^{1} \mathrm{P}^{\circ}, y^{3} \mathrm{P}^{\circ}, y^{1} \mathrm{P}^{\circ}$ \\
\hline $5 d 6 d$ & $f^{1} \mathrm{G}_{4}$ & $56,035.70$ & 806.27 & 1.03 & & 1.000 & $\begin{array}{c}z^{1} \mathrm{~F}^{\circ}, z^{1} \mathrm{G}^{\circ} \\
x^{\circ}, y^{3} \mathrm{~F}^{\circ}, z^{3} \mathrm{H}^{\circ}, z^{3} \mathrm{G}^{\circ}, z^{3} \mathrm{D}^{\circ}, y^{3} \mathrm{D}^{\circ}, x^{3} \mathrm{~F}^{\circ},\end{array}$ \\
\hline $5 d 6 d$ & $\begin{array}{l}g^{1} \mathrm{~N}_{2} \\
e^{1} \mathrm{~S}_{0}\end{array}$ & $55,184.05$ & & 1.08 & 1 & 1.000 & $\begin{array}{l}z^{3} \mathrm{G}^{\circ}, z^{3} \mathrm{D}^{\circ}, z^{3} \mathrm{P}^{\circ}, y^{3} \mathrm{D}^{\circ}, x^{3} \mathrm{~F}^{\circ}, y^{3} \mathrm{P}^{\circ} \\
z^{1} \mathrm{P}^{\circ}, z^{3} \mathrm{P}^{\circ}\end{array}$ \\
\hline $4 f^{2}$ & $\begin{array}{l}e^{3} \mathrm{H}_{4} \\
e^{3} \mathrm{H}_{5} \\
e^{3} \mathrm{H}_{6}\end{array}$ & $\begin{array}{l}55,107.25 \\
55,982.09 \\
56,837.94\end{array}$ & $\begin{array}{l}874.84 \\
855.85\end{array}$ & $\begin{array}{l}.94 \\
1.04 \\
1.18\end{array}$ & $\begin{array}{l}1 \\
2 \\
1\end{array}$ & $\begin{array}{l}.800 \\
1.033 \\
1.167\end{array}$ & $\left\{\begin{array}{l}z^{3} \mathrm{~F}^{\circ}, z^{1} \mathrm{~F}^{\circ}, z^{1} \mathrm{G}^{\circ}, z^{3} \mathrm{H}^{\circ}, z^{3} \mathrm{G}^{\circ}, z^{3} \mathrm{D}^{\circ}, y^{3} \mathrm{D}^{\circ}, x^{3} \mathrm{~F}^{\circ}, \\
.\end{array}\right.$ \\
\hline $4 f^{2}$ & $\begin{array}{l}g^{3} \mathrm{~F}_{2} \\
g^{3} \mathrm{~F}_{3} \\
g^{3} \mathrm{~F}_{4}\end{array}$ & $\begin{array}{l}57,399.58 \\
57,918.50 \\
58,259.41\end{array}$ & $\begin{array}{l}518.92 \\
340.91\end{array}$ & $\begin{array}{l}1.11 \\
1.22\end{array}$ & 1 & $\begin{array}{l}1.083 \\
1.250\end{array}$ & $\left\{\begin{array}{l}z^{3} \mathrm{~F}, z^{1} \mathrm{~F}^{\circ}, z^{1} \mathrm{G}^{\circ}, y^{3} \mathrm{~F}^{\circ}, z^{3} \mathrm{H}^{\circ}, z^{1} \mathrm{D}^{\circ}, z^{3} \mathrm{G}^{\circ}, z^{3} \mathrm{D}^{\circ}, \\
z^{3} \mathrm{P}^{\circ}, x^{1} \mathrm{~F}^{\circ}\end{array}\right.$ \\
\hline $\begin{array}{l}4 f^{2} \\
4 f^{2}\end{array}$ & $\begin{array}{l}g^{1} \mathrm{G}_{44}^{2} \\
h^{1} \mathrm{D}_{2}\end{array}$ & $\begin{array}{l}59,527.60 \\
59,900.08\end{array}$ & & $\begin{array}{l}1.07 \\
1.00\end{array}$ & 1 & $\begin{array}{l}1.000 \\
1.000\end{array}$ & $\begin{array}{l}z^{1} \mathrm{G}^{\circ}, y^{3} \mathrm{~F}^{\circ}, z^{3} \mathrm{H}^{\circ}, z^{3} \mathrm{G}^{\circ}, y^{1} \mathrm{~F}^{\circ}, z^{1} \mathrm{H}^{\circ}, x^{1} \mathrm{~F}^{\circ} \\
z^{3} \mathrm{~F}^{\circ}, z^{1} \mathrm{~F}^{\circ}, y^{3} \mathrm{~F}^{\circ}, z^{1} \mathrm{D}^{\circ}, z^{3} \mathrm{D}^{\circ}, z^{3} \mathrm{P}^{\circ}, y^{1} \mathrm{D}^{\circ}, y^{1} \mathrm{~F}^{\circ}, \\
y^{3} \mathrm{D}^{\circ}, x^{3} \mathrm{~F}^{\circ}, x^{1} \mathrm{~F}^{\circ} .\end{array}$ \\
\hline $6 p^{2}$ & $\begin{array}{l}f^{3} \mathrm{P}_{03} \\
f^{3} \mathrm{P}_{1}\end{array}$ & $60,094.84$ & $1,033.99$ & $\begin{array}{r}0 / 0 \\
1.47\end{array}$ & $11 / 2$ & $\begin{array}{r}0 / 0 \\
1.500\end{array}$ & $y^{3} \mathrm{~F}^{\circ}, z^{1} \mathrm{D}^{\circ}, z^{3} \mathrm{D}^{\circ}, z^{3} \mathrm{P}^{\circ}, y^{1} \mathrm{D}^{\circ}, y^{1} \mathrm{~F}^{\circ}, y^{3} \mathrm{D}^{\circ}, z^{1} \mathrm{P}^{\circ}$, \\
\hline $6 p^{2}$ & ${ }_{i 1}^{f^{3} \mathrm{P}_{2}}$ & $\begin{array}{l}62,506.36 \\
62,026.27\end{array}$ & $1,377.53$ & 1.45 & $11 / 2$ & 1. 500 & $\begin{array}{l}y^{3} \mathrm{P}^{\circ}, x^{3} \mathrm{P}^{\circ} \\
z^{1} \mathrm{~F}^{\circ}, y^{3} \mathrm{~F}^{\circ}, z^{1} \mathrm{D}^{\circ}, z^{3} \mathrm{D}^{\circ}, z^{3} \mathrm{P}^{\circ}, y^{1} \mathrm{D}^{\circ}, y^{1} \mathrm{~F}^{\circ}, x^{3} \mathrm{~F}^{\circ}, \\
z^{1} \mathrm{P}^{\circ}, y^{3} \mathrm{P}^{\circ}, y^{1} \mathrm{P}^{\circ}, x^{1} \mathrm{~F}^{\circ}, x^{1} \mathrm{P}^{\circ} .\end{array}$ \\
\hline $\begin{array}{l}4 f^{2} \\
4 f^{2}\end{array}$ & $\begin{array}{l}e^{1} \mathrm{I}_{6} \\
g^{3} \mathrm{P}_{0} \\
g^{3} \mathrm{P}_{1} \\
g^{3} \mathrm{P}_{2}\end{array}$ & $\begin{array}{l}62,408.40 \\
63,463.95 \\
63,703.18 \\
64,278.92\end{array}$ & $\begin{array}{l}239.23 \\
575.74\end{array}$ & 1.01 & 1 & 1.000 & $\left\{\begin{array}{l}z^{3} \mathrm{H}^{3}, z^{1} \mathrm{H}^{\circ} \\
z^{1} \mathrm{D}^{\circ}, z^{3} \mathrm{D}^{\circ}, z^{3} \mathrm{P}^{\circ}, y^{1} \mathrm{~F}^{\circ}, y^{3} \mathrm{D}^{\circ}, x^{3} \mathrm{~F}^{\circ}, z^{1} \mathrm{P}^{\circ}, y^{3} \mathrm{P}^{\circ}, \\
x^{3} \mathrm{P}^{\circ}\end{array}\right.$ \\
\hline $6 s 6 d$ & $\begin{array}{l}h^{3} \mathrm{D}_{1} \\
h^{3} \mathrm{D}_{2} \\
h^{3} \mathrm{D}_{3} \\
1 \mathrm{D}_{2} ?\end{array}$ & $\begin{array}{l}64,361.28 \\
64,529.90 \\
64,692.59 \\
64,706.76 ?\end{array}$ & $\begin{array}{l}168.62 \\
162.69\end{array}$ & & & & $\begin{array}{l}z^{1} \mathrm{D}^{\circ}, z^{3} \mathrm{P}^{\circ}, y^{3} \mathrm{D}^{\circ}, z^{1} \mathrm{P}^{\circ}, y^{3} \mathrm{P}^{\circ}, y^{1} \mathrm{P}^{\circ}, x^{3} \mathrm{P}^{\circ} \\
y^{1} \mathrm{P}^{\circ}, x^{1} \mathrm{~F}^{\circ}\end{array}$ \\
\hline $\begin{array}{c}6 p^{2} \\
4 f^{2} \\
6 d 6 s\end{array}$ & $\begin{array}{c}f^{1} \mathrm{~S}_{0} \\
g^{1} \mathrm{~S}_{0} \\
i^{3} \mathrm{D}_{3} \\
1_{3}^{10} \\
2 \\
2_{4}^{\circ} \\
3_{3,2}^{\circ} \\
4_{4}^{\circ} \\
5_{4,3}^{\circ} \\
6_{4}^{\circ}, 3 \\
7_{4,3}^{\circ}, 3 \\
8_{3}^{\circ}\end{array}$ & $\begin{array}{l}66,591.91 \\
69,505.06 \\
69,233.90 \\
57,364.12 \\
58,748.90 \\
59,612.64 \\
60,744.17 \\
61,017.66 \\
61,514.46 \\
63,598.87 \\
64,411.17\end{array}$ & & & & & $\begin{array}{l}z^{1} \mathrm{P}^{\circ}, y^{3} \mathrm{P}^{\circ}, y^{1} \mathrm{P}^{\circ} \\
z^{1} \mathrm{P}^{\circ}, y^{3} \mathrm{P}^{\circ}, y^{1} \mathrm{P}^{\circ} \\
y^{3} \mathrm{D}^{\circ}, x^{3} \mathrm{~F}^{\circ} \\
e^{3} \mathrm{G}, e^{3 \mathrm{~F}}, e^{3} \mathrm{D} \\
e^{3} \mathrm{G}, e^{3 \mathrm{~F}}, e^{1} \mathrm{~F} \\
e^{3} \mathrm{G}, e^{3} \mathrm{~F}, e^{3} \mathrm{D} \\
e^{3} \mathrm{G}, e^{3} \mathrm{D} \\
e^{3 \mathrm{~F}} . \\
e^{1 \mathrm{~F}}, e^{1} \mathrm{G} \\
e^{3} \mathrm{G}, e^{1} \mathrm{G} \\
e^{3} \mathrm{G}, e^{1} \mathrm{~F}, e^{3} \mathrm{D}\end{array}$ \\
\hline
\end{tabular}




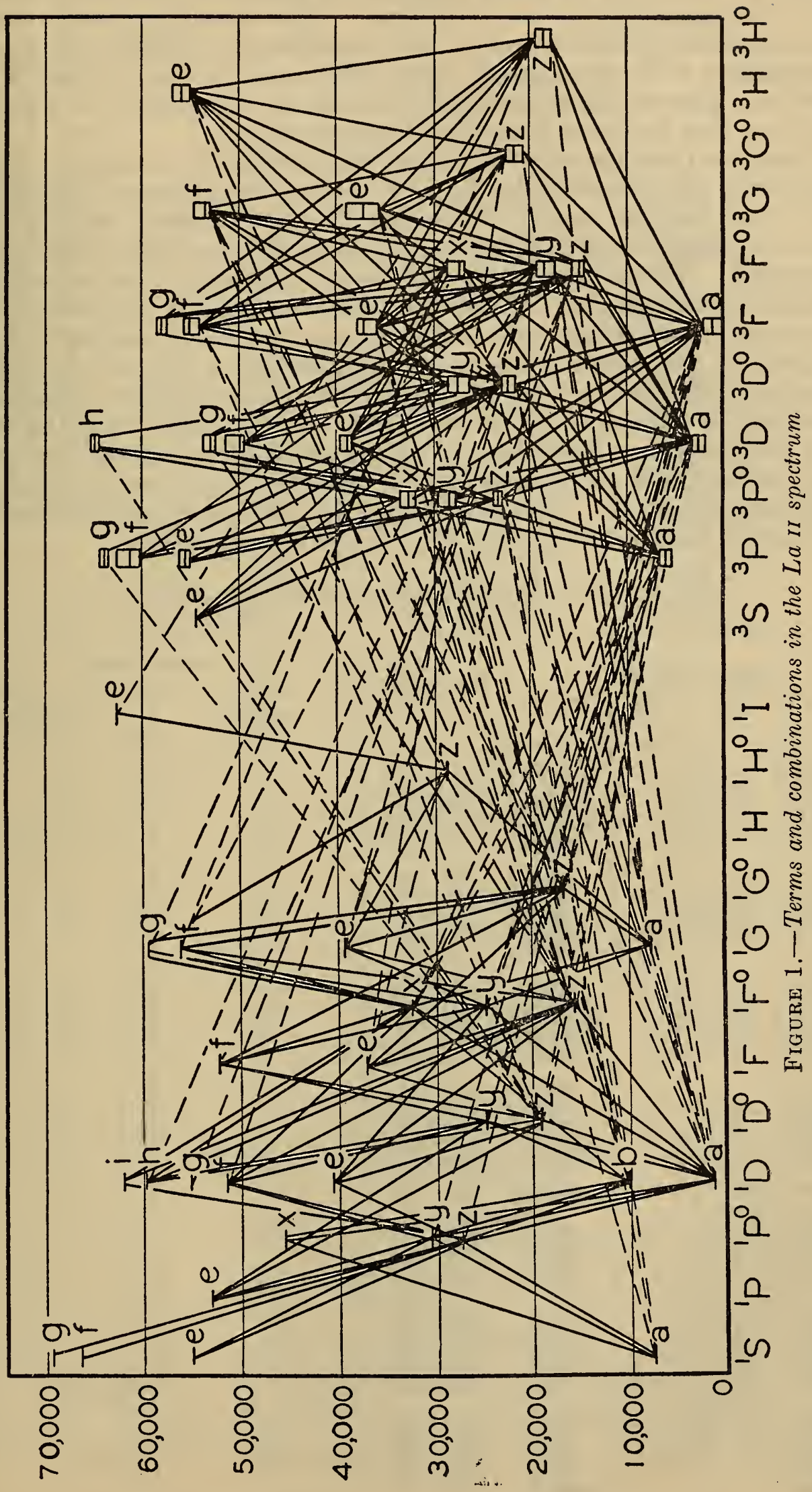


A diagram of the La II terms and combinations is reproduced in Figure 1. In order to avoid making the figure too confusing some of the combinations have been omitted. The abundance and strength of intersystem connections is very striking in lanthanum spectra. Comparison with the corresponding diagrams for Sc ${ }^{23}$ and $\mathrm{Y} \mathrm{II}^{24}$ show at a glance the remarkable increase in complexity of the La II spectrum due to terms involving the $f$-electron.

Complete data for all of the observed lines characteristic of ionized lanthanum are presented in Table 4, successive columns of which contain wave lengths, spark intensities, furnace classes, vacuum wave numbers, term combinations, and Zeeman effects, both observed and computed. The latter are derived from the adopted $g$ values with the formulas given above. When the observed pattern is resolved, the computed components are given separately; when unresolved, the centroid of the blend given by equations (2) or (4), except for the patterns described as $A^{1}$ or $A^{2}$, where the strongest component according to equation (1) is tabulated. In the latter case the measured position often deviates a little toward the centroid of the group. Apart from this the agreement of the observed and computed values is usually satisfactory. One faint line, 4,193.34 A is entirely discordant, and clearly does not arise from the assigned combination.

TABLE 4.-The first spark spectrum of lanthanum (La II)

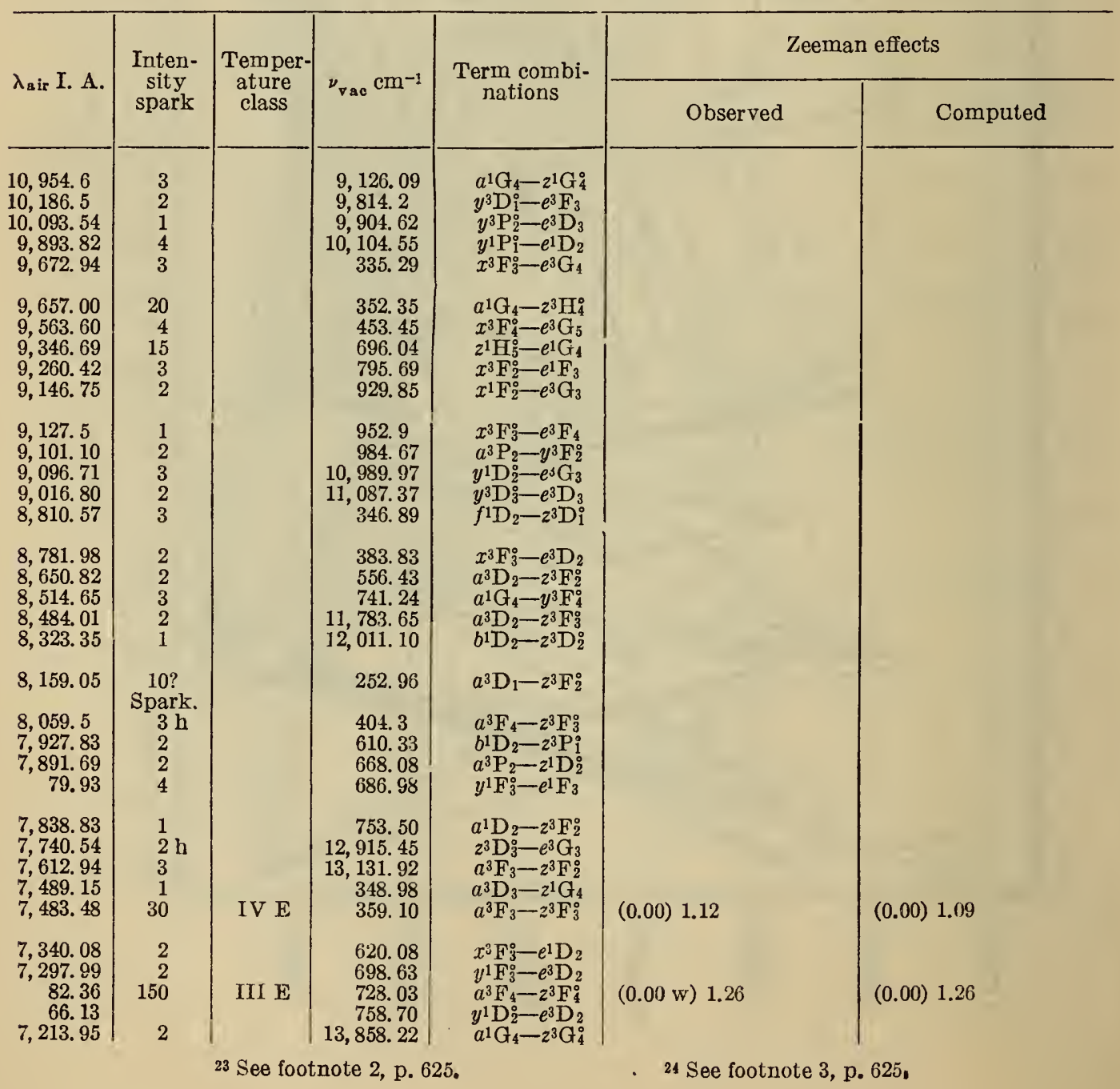


TABLE 4.-The first spark spectrum of lanthanum (La II)-Continued

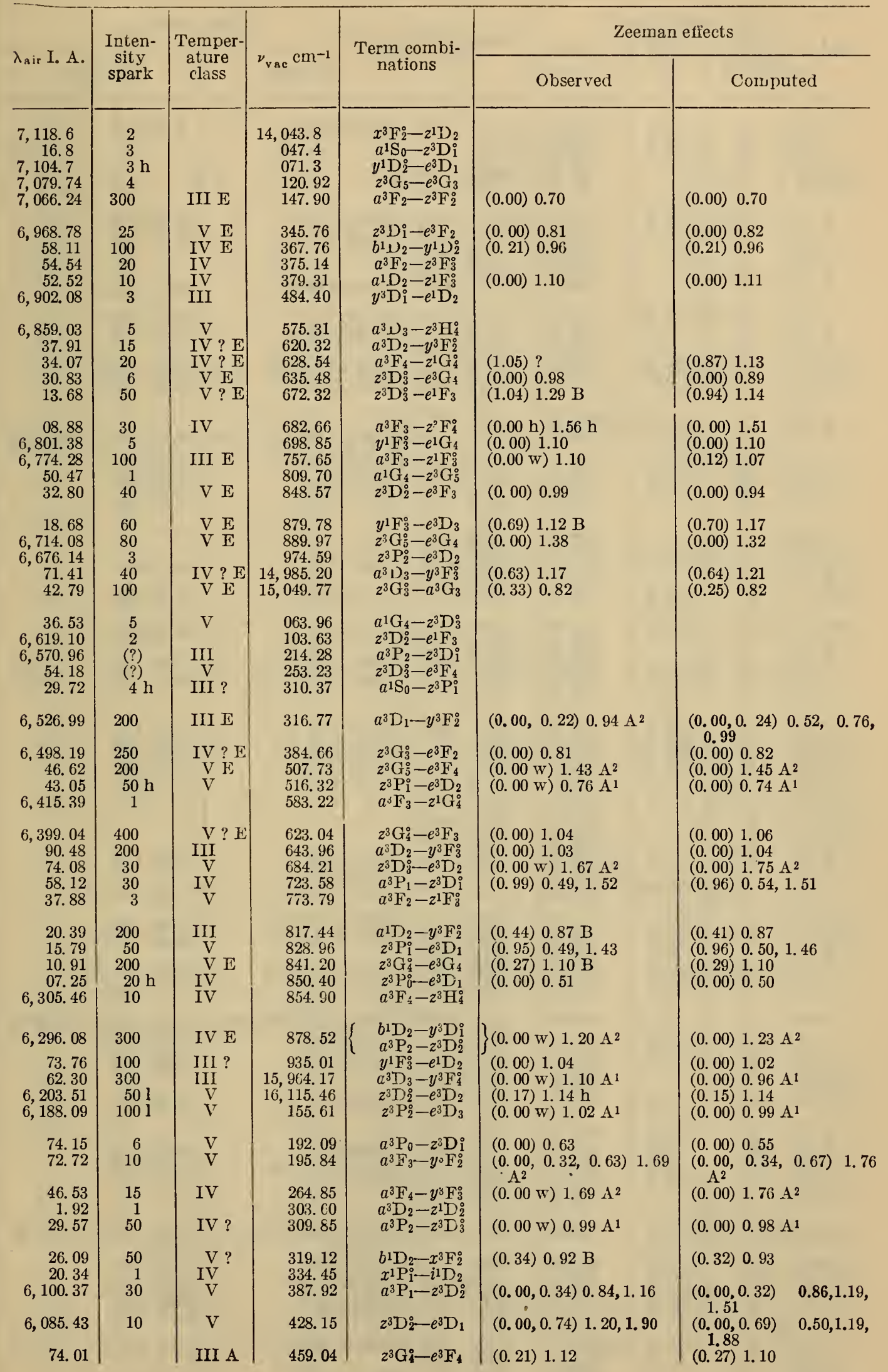


TABLE 4.-The first spark spectrum of lanthanum (La II)-Continued

\begin{tabular}{|c|c|c|c|c|c|c|}
\hline \multirow{2}{*}{$\lambda_{\mathrm{air}}$ I. A. } & \multirow{2}{*}{$\begin{array}{l}\text { Inten- } \\
\text { sity } \\
\text { spark }\end{array}$} & \multirow{2}{*}{$\begin{array}{c}\text { Temper- } \\
\text { ature } \\
\text { class }\end{array}$} & \multirow{2}{*}{$\nu_{\mathrm{VBC}} \mathrm{cm}^{-1}$} & \multirow{2}{*}{$\begin{array}{l}\text { Term combi- } \\
\text { nations }\end{array}$} & \multicolumn{2}{|c|}{ Zeeman effects } \\
\hline & & & & & Observed & Computed \\
\hline $\begin{array}{r}67.13 \\
61.42 \\
46.07 \\
6,037.98 \\
5,991.98\end{array}$ & $\begin{array}{l}6 \\
2 \\
2 \\
2 \\
4 \mathrm{~h}\end{array}$ & $\begin{array}{l}\text { V? } \\
\text { V? } \\
\text { IV? }\end{array}$ & $\begin{array}{l}477.71 \\
493.23 \\
535.10 \\
557.25 \\
684.36\end{array}$ & $\begin{array}{l}a^{3} \mathrm{P}_{2}-z^{3} \mathrm{P}_{\mathrm{i}}^{\circ} \\
2^{1} \mathrm{D}_{2}^{\circ}-e^{3} \mathrm{G}_{3} \\
2^{3} \mathrm{D}_{3}^{0}-e^{1} \mathrm{G}_{4}\end{array}$ & $(0.00) 1.49$ & $(0.00) 1.50$ \\
\hline $\begin{array}{l}73.52 \\
71.09 \\
61.43 \\
57.90 \\
48.30\end{array}$ & $\begin{array}{c}1201 \\
8 \\
3 \\
4 \\
20\end{array}$ & $\mathrm{VE}$ & $\begin{array}{l}735.92 \\
742.73 \\
769.86 \\
779.80 \\
806.9\end{array}$ & $\begin{array}{l}z^{3} \mathrm{G}_{5}^{\circ}-e^{3} \mathrm{G}_{5} \\
b^{1} \mathrm{D}_{2}-x^{3} \mathrm{~F}_{3}^{\circ} \\
z^{3} \mathrm{G}_{3}^{\circ}-e^{3} \mathrm{G}_{4} \\
z^{3} \mathrm{D}_{1}^{\circ}-e^{3} \mathrm{D}_{2} \\
z^{3} \mathrm{G}_{3}^{\circ}-e^{1} \mathrm{~F}_{3}\end{array}$ & $\begin{array}{l}(0.00) 1.20 \\
(0.00) 1.18 \\
(0.52) 0.86 \mathrm{~h}\end{array}$ & $\begin{array}{l}(0.00) 1.20 \\
(0.00) 1.15 \\
(0.46) 0.86\end{array}$ \\
\hline $\begin{array}{r}36.22 \\
27.71 \\
18.26 \\
5,901.95 \\
5,892.66\end{array}$ & $\begin{array}{c}20 \\
30 \\
4 \\
401 \\
4\end{array}$ & $\begin{array}{l}\text { V E } \\
\text { IV E }\end{array}$ & $\begin{array}{l}841.08 \\
865.26 \\
892.19 \\
938.87 \\
965.58\end{array}$ & $\begin{array}{l}a^{1} \mathrm{D}_{2}-y^{3} \mathrm{~F}_{3}^{\circ} \\
z^{3} \mathrm{D}_{3}^{\circ}-e^{3} \mathrm{D}_{3} \\
z^{1} \mathrm{D}_{2}-e^{3} \mathrm{~F}_{2} \\
z^{3} \mathrm{G}_{5}^{\circ}-e^{1} \mathrm{G}_{4} \\
a^{3} \mathrm{P}_{1}-z^{3} \mathrm{P}_{0}\end{array}$ & $\begin{array}{l}(0.00) 1.21 \\
(0.00) 1.30 \\
(0.00 \mathrm{w}) 1.60 \mathrm{~A}^{2} \\
(0.00) 1.54\end{array}$ & $\begin{array}{l}(0.00) 1.19 \\
(0.03) 1.31 \\
(0.00) 1.78 \mathrm{~A}^{2} \\
(0.00) 1.51\end{array}$ \\
\hline $\begin{array}{l}85.23 \\
80.63\end{array}$ & $\begin{array}{r}1 \\
50\end{array}$ & III E ? & $\begin{array}{l}16,986.99 \\
17,000.28\end{array}$ & $\begin{array}{l}a^{3} \mathrm{P}_{1}-z^{3} \mathrm{P}_{1}^{\circ} \\
a^{3} \mathrm{D}_{1}-z^{1} \mathrm{D}_{2}^{\circ}\end{array}$ & $(0.00,0.42) \quad 0.54, \quad 0.96$ & $(0.00,0.41) \quad 0.52,0.93$ \\
\hline $\begin{array}{l}74.00 \\
63.70 \\
48.95\end{array}$ & $\begin{array}{r}6 \\
80 \\
20\end{array}$ & $\mathrm{IV}_{\mathrm{V}}$ & $\begin{array}{l}019.47 \\
049.36 \\
092.36\end{array}$ & $\begin{array}{l}a^{3} \mathrm{P}_{2}-z^{3} \mathrm{P}_{2}^{\circ} \\
a^{1} \mathrm{G}_{4}-y^{1} \mathrm{~F}_{3}^{\circ} \\
z^{3} \mathrm{D}_{1}^{\circ}-e^{3} \mathrm{D}_{1}\end{array}$ & $\begin{array}{l}(0.00) 1.49 \\
(0.00) 0.96 \\
(0.00) 0.54\end{array}$ & 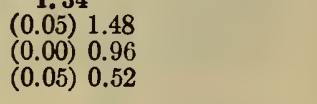 \\
\hline $\begin{array}{r}28.44 \\
08.63 \\
08.31 \\
06.56 \\
5,805.77\end{array}$ & $\begin{array}{r}2 \\
8 \\
60 \\
8 \\
120\end{array}$ & $\begin{array}{l}\text { IV ? } \\
\text { III E }\end{array}$ & $\begin{array}{l}152.51 \\
211.00 \\
211.95 \\
217.14 \\
219.48\end{array}$ & $\begin{array}{l}a^{3} \mathrm{D}_{3}-z^{3} \mathrm{G}_{3}^{\circ} \\
2^{3} \mathrm{P}_{2}^{\circ}-e^{1} \mathrm{D}_{2} \\
a^{3} \mathrm{~F}_{2}-y^{3} \mathrm{~F}_{2}^{\circ} \\
y^{3} \mathrm{~F}_{3}^{\circ}-e^{3} \mathrm{G}_{3} \\
a^{3} \mathrm{~F}_{3}-y^{3} \mathrm{~F}_{3}^{\circ}\end{array}$ & $\begin{array}{l}(0.00) 0.75 \\
(0.00) 1.09\end{array}$ & $\begin{array}{l}(0.05) 0.74 \\
(0.00) 1.09\end{array}$ \\
\hline $\begin{array}{r}5,797.57 \\
81.02 \\
79.91 \\
69.06 \\
49.59\end{array}$ & $\begin{array}{r}150 \\
3 \\
4 \\
60 \\
2\end{array}$ & $\mathrm{VE}$ & $\begin{array}{l}243.84 \\
293.20 \\
296.52 \\
329.05 \\
387.73\end{array}$ & $\begin{array}{l}a^{3} \mathrm{~F}_{4}-y^{3} \mathrm{~F}_{4}^{\circ} \\
b^{1} \mathrm{D}_{2}-y^{3} \mathrm{D}_{2}^{\circ} \\
z^{3} \mathrm{D}_{2}^{\circ}-e^{3} \mathrm{D}_{3} \\
b^{1} \mathrm{D}_{2}-z^{1} \mathrm{P}_{1}^{\circ} \\
z^{3} \mathrm{G}_{3}^{\circ}-e^{3} \mathrm{~F}_{4}^{4}\end{array}$ & $\begin{array}{l}(0.00) 1.24 \\
(0.00) 1.38 \mathrm{~h} \\
(0.00) 1.09\end{array}$ & $\begin{array}{l}(0.05) 1.25 \\
(0.00) 1.43 \\
(0.00) 1.08\end{array}$ \\
\hline $\begin{array}{r}27.29 \\
12.39 \\
5,703.32 \\
5,671.54 \\
52.3\end{array}$ & $\begin{array}{c}20 \\
20 \\
20 \\
100 \\
10 \mathrm{~h}\end{array}$ & $\begin{array}{l}\text { V E } \\
\text { III E ? } \\
\text { III } \\
\text { V E }\end{array}$ & $\begin{array}{l}455.43 \\
500.96 \\
528.80 \\
627.02 \\
687.0\end{array}$ & $\begin{array}{l}a^{3} \mathrm{P}_{0}-z^{3} \mathrm{P}_{1}^{\circ} \\
a^{1} \mathrm{D}_{2}-z^{1} \mathrm{D}_{2}^{\circ} \\
a^{3} \mathrm{P}_{1}-z^{3} \mathrm{P}_{2} \\
z^{3} \mathrm{H}_{4}^{\circ}-e^{0} \mathrm{G}_{3} \\
z^{3} \mathrm{G}_{4}^{\circ}-e^{3} \mathrm{G}_{5}\end{array}$ & $\begin{array}{ll}(0.00) & 1.45 \\
(0.00) & 0.98 \\
(0.00) & 1.48 \\
(0.00) & 0.79\end{array}$ & $\begin{array}{ll}(0.00) & 1.46 \\
(0.10) & 0.96 \\
(0.00) & 1.44 \\
(0.00) & 0.85\end{array}$ \\
\hline $\begin{array}{r}5,610.53 \\
5,591.51 \\
66.92 \\
47.56 \\
35.66\end{array}$ & $\begin{array}{l}20 \\
1 \\
40 \\
3 \mathrm{~h} \\
80\end{array}$ & $\begin{array}{l}\text { V E } \\
\text { V E }\end{array}$ & $\begin{array}{r}818.69 \\
879.30 \\
17,958.28 \\
18,020.95 \\
059.69\end{array}$ & $\begin{array}{l}z^{3} \mathrm{G}_{3}^{\circ}-e^{3} \mathrm{D}_{2} \\
a^{3} \mathrm{~F}_{3}-z^{1} \mathrm{D}_{2}^{\circ} \\
y^{3} \mathrm{~F}_{1}^{\circ}-e^{3} \mathrm{G}_{4} \\
b^{1} \mathrm{D}_{2}-y^{3} \mathrm{P}_{1}^{\circ}\end{array}$ & $\begin{array}{l}(0.37) 1.24 \\
(0.00) 1.13 \\
(0.00 \mathrm{w}) 0.80 \mathrm{~A}^{1}\end{array}$ & $\begin{array}{l}(0.32) 1.20 \\
(0.00) 0.77 \mathrm{~A}^{1}\end{array}$ \\
\hline $\begin{array}{r}5,532.17 \\
5,493.45 \\
86.86 \\
82.27 \\
80.72\end{array}$ & $\begin{array}{r}10 \\
20 \\
5 \\
40 \\
25\end{array}$ & $\begin{array}{l}\text { III } \\
\text { V E } \\
\text { V } \\
\text { V E }\end{array}$ & $\begin{array}{l}071.08 \\
198.45 \\
220.31 \\
235.57 \\
240.72\end{array}$ & $\begin{array}{l}z^{3} \mathrm{G}_{4}^{\circ}-e^{3} \mathrm{D}_{3} \\
a^{3} \mathrm{~F}_{3}-y^{3} \mathrm{~F}_{4}^{\circ} \\
b^{1} \mathrm{D}_{2}-y^{3} \mathrm{D}_{3}^{\circ} \\
a^{3} \mathrm{~F}_{2}-y^{3} \mathrm{~F}_{3}^{\circ} \\
y^{3} \mathrm{~F}_{2}^{\circ}-e^{3} \mathrm{G}_{3}\end{array}$ & $\begin{array}{l}(0.00 \mathrm{w}) 0.61 \mathrm{~h} \\
(0.00 \mathrm{w}) 1.57 \mathrm{~A}^{2} \\
(0.00 \mathrm{w}) 1.89 \mathrm{~A}^{2} \\
(0.00) 1.03 \mathrm{~h}\end{array}$ & 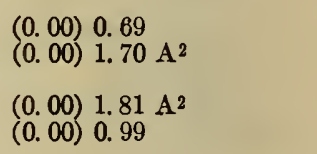 \\
\hline 64.37 & 25 & $\mathrm{VE}$ & 295.30 & $a^{3} \mathrm{P}_{2}-y^{1} \mathrm{~F}_{3}^{\circ}$ & $(0.00,0.49,0.97) 0.00 \mathrm{~d}$ & $(0.00,0.46,0.91) \quad 0.12$, \\
\hline $\begin{array}{r}58.68 \\
47.59 \\
5,423.82 \\
5,381.91\end{array}$ & $\begin{array}{r}50 \\
10 \\
4 \\
100\end{array}$ & $\mathrm{VE}$ & $\begin{array}{l}314.37 \\
351.65 \\
432.08 \\
575.61\end{array}$ & $\begin{array}{l}z^{1} \mathrm{D}_{2}^{0}-e^{1} \mathrm{~F}_{3} \\
2^{3} \mathrm{D}_{2}^{0}-e^{1} \mathrm{D}_{2} \\
a^{3} \mathrm{~F}_{4}-z^{3} \mathrm{G}_{3}^{\circ} \\
y^{3} \mathrm{~F}_{2}^{0}-e^{3} \mathrm{~F}_{2}\end{array}$ & $\begin{array}{l}(0.00) 1.00 \\
(0.22) 1.14 \\
(0.00) 0.74\end{array}$ & $\begin{array}{l}(0.00) 0.98 \\
(0.25) 1.12 \\
(0.00) 0.72\end{array}$ \\
\hline $\begin{array}{l}81.77 \\
80.97 \\
77.08 \\
40.66 \\
33.42\end{array}$ & $\begin{array}{r}50 \\
100 \\
200 \\
100 \\
2\end{array}$ & $\begin{array}{l}\text { V E } \\
\text { V } \\
\text { V } \\
\text { III } \\
\text { E }\end{array}$ & $\begin{array}{l}576.10 \\
578.86 \\
592,30 \\
719.09 \\
744.50\end{array}$ & $\begin{array}{l}z^{3} \mathrm{~F}_{4}^{\circ}-e^{3} \mathrm{~F}_{4} \\
a^{1} \mathrm{~S}_{0}-y^{3} \mathrm{D}_{1}^{\circ} \\
2^{3} \mathrm{H}_{5}^{\circ}-e^{3} \mathrm{G}_{4} \\
y^{3} \mathrm{~F}_{3}^{\circ}-e^{3} \mathrm{~F}_{3} \\
a^{3} \mathrm{P}_{1}-y^{1} \mathrm{D}_{2}\end{array}$ & $\begin{array}{l}(0.00) 0.77 \\
(0.00 \mathrm{w}) 0.82 \mathrm{~A}^{1} \\
(0.00) 1.07\end{array}$ & $\begin{array}{l}(0.00) 0.80 \\
(0.00) 0.83 \mathrm{~A}^{1} \\
(0.08) 1.08\end{array}$ \\
\hline 03.54 & 100 & III E & 850.10 & $\dot{a}^{3} \mathrm{D}_{2}-z^{3} \mathrm{D}_{\mathrm{i}}^{\circ}$ & $\begin{array}{l}(0.00,0.61) 0.57,1.17, \\
1.78\end{array}$ & $\begin{array}{c}(0.00,0.59) \\
1.73\end{array}$ \\
\hline $\begin{array}{r}02.62 \\
5,301.97 \\
5,290.83 \\
79.11\end{array}$ & $\begin{array}{r}150 \\
200 \\
50 \\
40\end{array}$ & $\underset{\text { III }}{\operatorname{II}} \underset{\mathrm{E}}{\mathrm{E}}$ & $\begin{array}{r}853.37 \\
855.68 \\
895.38 \\
18,937.33\end{array}$ & $\begin{array}{l}z^{1} \mathrm{G}_{1}^{\circ}-e^{3} \mathrm{G}_{3} \\
a^{3} \mathrm{D}_{3}-z^{3} \mathrm{D}_{2}^{\circ} \\
a^{3} \mathrm{~F}_{2}-z^{1} \mathrm{D}_{2}^{\circ} \\
y^{3} \mathrm{~F}_{3}-e^{3} \mathrm{G}_{1}\end{array}$ & $\begin{array}{l}(0.00) 1.16 \\
(0.00 \mathrm{~W}) 1.50 \mathrm{~W} \\
(0.34) 0.82 \mathrm{~B} \\
(0.00) 1.20\end{array}$ & $\begin{array}{l}(0.00) 1.18 \\
(0.00) 1.49 \\
(0.36) 0.83 \\
(0.00) 1.23\end{array}$ \\
\hline $\begin{array}{l}59.38 \\
57.28 \\
26.20 \\
22.48 \\
21.32\end{array}$ & $\begin{array}{c}50 \\
2 \\
40 \mathrm{l} \\
3 \mathrm{~h} \\
3 \mathrm{~h}\end{array}$ & $\begin{array}{r}\text { III E } \\
\text { V E }\end{array}$ & $\begin{array}{r}19,008.37 \\
015.96 \\
129.05 \\
142.68 \\
146.93\end{array}$ & $\begin{array}{l}a^{1} \mathrm{D}_{2}-z^{3} \mathrm{G}_{3}^{\circ} \\
z^{3} \mathrm{D}_{1}^{\circ}-e^{1} \mathrm{D}_{2} \\
2^{3} \mathrm{H}_{4}^{\circ}-e^{3} \mathrm{~F}_{3} \\
e^{3} \perp_{2}-1_{3}^{\circ}\end{array}$ & $\begin{array}{l}(0.00 \mathrm{w}) 0.46 \mathrm{~A}^{1} \\
(0.00 \mathrm{w}) 0.32 \mathrm{~W} \\
(0.00) 1.15\end{array}$ & $\begin{array}{l}(0.00) 0.35 \mathrm{~A}^{1} \\
(0.00) 0.28 \mathrm{~A}^{1}\end{array}$ \\
\hline
\end{tabular}


TABLE 4.-The first spark spectrum of lanthanum (La II)-Continued

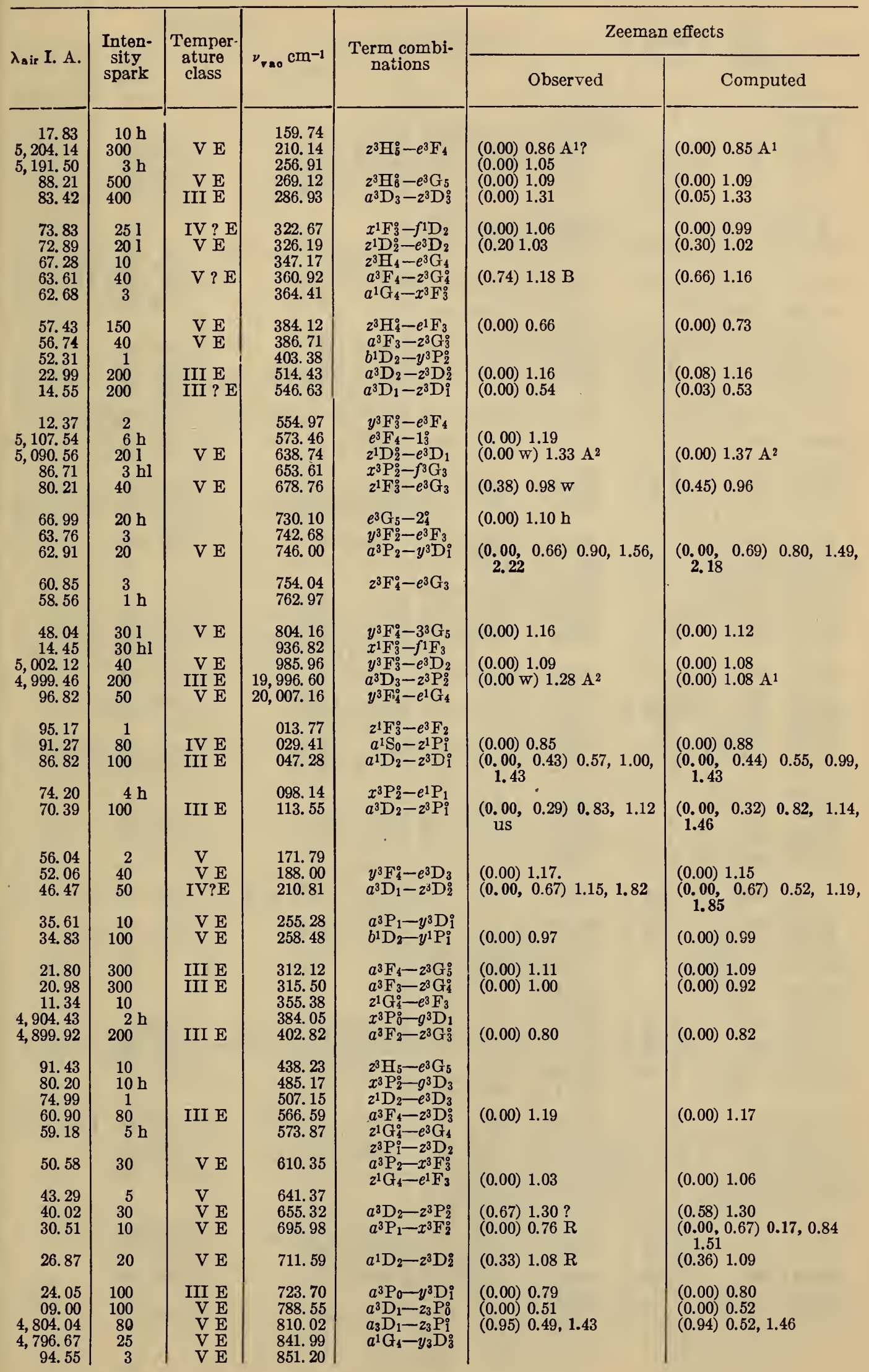


TABLE 4.-The first spark spectrum of lanthanum (La II)-Continued

\begin{tabular}{|c|c|c|c|c|c|c|}
\hline \multirow{2}{*}{$\lambda_{\text {sir I. A. }}$} & \multirow{2}{*}{$\begin{array}{l}\text { Inten- } \\
\text { sity } \\
\text { spark }\end{array}$} & \multirow{2}{*}{$\begin{array}{l}\text { Temper- } \\
\text { ature } \\
\text { class }\end{array}$} & \multirow{2}{*}{$\nu_{\mathrm{VAO}} \mathrm{cm}^{-1}$} & \multirow{2}{*}{$\begin{array}{l}\text { Term combi- } \\
\text { nations }\end{array}$} & \multicolumn{2}{|c|}{ Zeeman effects } \\
\hline & & & & & Observed & Computed \\
\hline $\begin{array}{l}80.55 \\
58.40 \\
48.73 \\
43.08 \\
40.27\end{array}$ & $\begin{array}{r}2 \\
3 \\
150 \\
250 \\
120\end{array}$ & $\begin{array}{l}\mathrm{V} \\
\mathrm{V} \\
\mathrm{V} \\
\mathrm{III} \\
\mathrm{E}\end{array}$ & $\begin{array}{r}20,912.27 \\
21,009.61 \\
052.39 \\
077.47 \\
089.96\end{array}$ & $\begin{array}{c}y^{3} \mathrm{~F}_{2}^{\circ}-e^{3} \mathrm{D}_{2} \\
a^{1} \mathrm{G}_{4}-z^{1} \mathrm{H}_{5}^{\circ} \\
z^{3} \mathrm{~F}_{3}^{\circ}-e^{3} \mathrm{G}_{3} \\
a^{3} \mathrm{~F}_{3}-z^{3} \mathrm{D}_{2}^{\circ}\end{array}$ & $\begin{array}{l}(0.00) 1.00 \\
(0.52 \mathrm{w}) 0.95 \mathrm{~B} \\
(0.00 \mathrm{w}) 0.97 . \mathrm{A}^{1}\end{array}$ & $\begin{array}{l}(0.00) 0.99 \\
(0.55) 0.98 \\
(0.00) 0.90 \mathrm{~A}^{1}\end{array}$ \\
\hline $\begin{array}{l}39.80 \\
30.73 \\
28.41\end{array}$ & $\begin{array}{c}15 \\
3 \mathrm{~h} \\
100\end{array}$ & $\begin{array}{l}\text { V E } \\
\text { V E }\end{array}$ & $\begin{array}{l}092.06 \\
132.50 \\
142.86\end{array}$ & $\begin{array}{l}a^{1} G_{4}-x^{3} F_{4} \\
x^{1} \mathrm{~F}_{3}^{\circ}-f^{3} G_{4} \\
a^{1} D_{2}-2^{3} D_{3}^{\circ}\end{array}$ & $(0.00,0.32) 1.31,1.61$, & $0.00,0.33,0.66)-,-$, \\
\hline $\begin{array}{l}24.42 \\
22.14\end{array}$ & $\begin{array}{l}40 \\
2 \mathrm{~h}\end{array}$ & V E & $\begin{array}{l}160.72 \\
170.94\end{array}$ & $\begin{array}{l}a^{3} \mathrm{P}_{2}-y^{3} \mathrm{D}_{2}^{\circ} \\
y^{1} \mathrm{P}_{1}^{\circ}-f^{1} \mathrm{D}_{2}\end{array}$ & $\begin{array}{l}1.91 \\
(0.65) 0.95 \text { us }\end{array}$ & 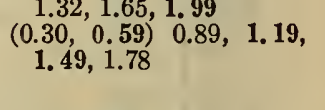 \\
\hline $\begin{array}{l}19.93 \\
17.58 \\
16.44\end{array}$ & $\begin{array}{r}150 \\
50 \\
80\end{array}$ & $\begin{array}{l}\text { V E } \\
\text { V E } \\
\text { V E }\end{array}$ & $\begin{array}{l}180.85 \\
191.40 \\
196.52\end{array}$ & $\begin{array}{l}z^{1} \mathrm{~F}_{3}^{\circ}-e^{3} \mathrm{~F}_{3} \\
z^{1} \mathrm{G}_{4}^{\circ}-e^{3} \mathrm{~F}_{4} \\
a^{3} \mathrm{P}_{2}-2^{1} \mathrm{P}_{1}^{\circ}\end{array}$ & $\begin{array}{l}(0.00) 1.06 \\
(0.50) 1.2 \\
(0.00,0.60) 0.92,1.52\end{array}$ & $\begin{array}{llll}(0.00) & 1.05 \\
(0.48) & 1.07 & & \\
(0.00, & 0.61) & 0.88, & 1.49\end{array}$ \\
\hline $4,703.27$ & 150 & $\mathrm{VE}$ & $\begin{array}{l}212.35 \\
255.87\end{array}$ & $\begin{array}{l}a^{3} \mathrm{D}_{3}-y^{1} \mathrm{D}_{2}^{\circ} \\
2^{3} \mathrm{~F}_{4}^{\circ}-e^{3} \mathrm{~F}_{3}\end{array}$ & $\begin{array}{l}2.12 \\
(0.00,0.47,0.92) 1.83 \\
2.26 \mathrm{R} \\
(0.00 \mathrm{w}) 1.70 \mathrm{~A}^{2}\end{array}$ & $\begin{array}{l}2.09 \\
(0.00,0.44,0.88)-,-,-, \\
1.78,2.22 \\
(0.00) 1.85 \mathrm{~A}^{2}\end{array}$ \\
\hline $\begin{array}{r}4,699.62 \\
92.50 \\
91.17\end{array}$ & $\begin{array}{r}50 \\
200 \\
50\end{array}$ & $\begin{array}{l}\mathrm{V} \\
\mathrm{V} \\
\mathrm{V}\end{array}$ & $\begin{array}{l}272.38 \\
304.66 \\
310.70\end{array}$ & $\begin{array}{r}a^{3} \mathrm{D}_{3}-y^{1} \mathrm{~F}_{3}^{\circ} \\
z^{3} \mathrm{~F}_{2}^{\circ}-e^{3} \mathrm{G}_{3} \\
a^{1} \mathrm{D}_{2}-z^{3} \mathrm{P}_{1}^{\circ}\end{array}$ & $\begin{array}{l}(0.00 \mathrm{w}) 1.21 \mathrm{~A}^{2} \\
(0.00,0.46) 0.46 \text { us }\end{array}$ & $\begin{array}{l}(0.00) 1.26 \mathrm{~A}^{2} \\
(0.00,0.47) 0.51,0.99\end{array}$ \\
\hline $\begin{array}{l}88.65 \\
84.39\end{array}$ & $\stackrel{40}{2 \mathrm{~h}}$ & V E & $\begin{array}{l}322.15 \\
341.54\end{array}$ & $\begin{array}{l}y^{3} \mathrm{~F}_{2}^{\circ}-e^{3} \mathrm{D}_{1} \\
e^{3} \mathrm{D}_{3}-4_{4}^{\circ}\end{array}$ & $(0.00) 0.96$ & $(0.00) 0.89$ \\
\hline $\begin{array}{l}82.12 \\
73.53 \\
71.82\end{array}$ & $\begin{array}{c}5 \\
1 \mathrm{~h} \\
200\end{array}$ & V E & $\begin{array}{l}351.89 \\
391.14 \\
398.96\end{array}$ & $\begin{array}{l}a^{3} \mathrm{D}_{1}-23 \mathrm{P}_{2}^{\circ} \\
e^{3} \mathrm{D}_{2}-3_{3}^{\circ}, 2 \\
2^{1} \mathrm{~F}_{3}^{\circ}-e^{3} \mathrm{G}_{4}\end{array}$ & $(0.00 \mathrm{w}) 1.29 \mathrm{~h}$ & $(0.00) 1.30$ \\
\hline 68.91 & 250 & $\begin{array}{l}\mathrm{VE} \\
\mathrm{VE}\end{array}$ & $\begin{array}{l}412.30 \\
435.94\end{array}$ & $\begin{array}{l}z^{3} \mathrm{~F}_{3}^{\circ}-e^{3} \mathrm{~F}_{2} \\
z^{1} \mathrm{~F}_{3}^{\circ}-e^{1} \mathrm{~F}_{3}\end{array}$ & $\begin{array}{l}(0.00,0.34,0.68) 0.77 \\
1.121 .47,1.81 \text { us } \\
(0.20) 0.99\end{array}$ & $\begin{array}{l}(0.00,0.36,0.71)-,-, \\
0.73,1.09,1.44,1.80 \\
(0.24) 0.99\end{array}$ \\
\hline $\begin{array}{l}62.51 \\
55.49 \\
52.07\end{array}$ & $\begin{array}{l}200 \\
400 \\
30 \mathrm{hl}\end{array}$ & $\underset{\mathrm{I}}{\operatorname{III}} \underset{\mathrm{E}}{\mathrm{E}}$ & $\begin{array}{l}441.69 \\
474.02 \\
489.81\end{array}$ & $\begin{array}{l}a^{3} \mathrm{~F}_{2}-z^{3} \mathrm{D}_{1}^{\circ} \\
z^{3} \mathrm{~F}_{4}^{\circ}-e^{3} \mathrm{G}_{4}\end{array}$ & $\begin{array}{l}(0.00 \mathrm{w}) 0.89 \mathrm{~A}^{2} \\
(0.43) 1.20 \mathrm{~B}\end{array}$ & $\begin{array}{l}(0.00) 0.91 \mathrm{~A}^{2} \\
(0.37) 1.20\end{array}$ \\
\hline $\begin{array}{l}47.50 \\
45.28\end{array}$ & $\begin{array}{l}100 \\
100\end{array}$ & $\begin{array}{ll}\text { V } & \mathrm{E} \\
\mathrm{V} & \mathrm{E}\end{array}$ & $\begin{array}{l}510.94 \\
521.22\end{array}$ & $\begin{array}{l}z^{3} \mathrm{~F}_{4}^{\circ}-e^{1} \mathrm{~F}_{3} \\
a^{3} \mathrm{~F}_{3}-z^{3} \mathrm{D}_{3}^{\circ}\end{array}$ & $\begin{array}{l}(0.00,0.31,0.61) 1.98 \mathrm{~A}^{2} \\
(0.60) 1.26 \mathrm{~B}\end{array}$ & $\begin{array}{l}(0.00,0.30,0.61) 2.17 \mathrm{~A}^{2}, \\
(0.58) 1.20\end{array}$ \\
\hline $\begin{array}{l}41.40 \\
36.42 \\
34.95 \\
23.99 \\
19.87\end{array}$ & $\begin{array}{c}2 \mathrm{~h} \\
80 \\
25 \mathrm{l} \\
2 \mathrm{~h} \\
300\end{array}$ & V E & $\begin{array}{l}539.21 \\
562.35 \\
569.19 \\
620.31 \\
639.59\end{array}$ & $\begin{array}{l}e^{1} \mathrm{~F}_{3}-2_{1}^{\circ} \\
2^{1} \mathrm{D}_{2}^{\circ}-e^{1} \mathrm{D}_{2} \\
y^{3} \mathrm{D}_{3}^{\circ}-f^{3} \mathrm{D}_{2} \\
2^{3} \mathrm{~F}_{2}^{\circ}-e^{3} \mathrm{~F}_{2}\end{array}$ & $\begin{array}{l}(0.21) 1.00 \\
(0.00) 1.43 \\
(0.00) 0.70\end{array}$ & $\begin{array}{ll} & \\
(0.21) & 0.99 \\
(0.00) & 1.48 \\
(0.10) & 0.70\end{array}$ \\
\hline 13.38 & 200 & $\mathrm{VE}$ & 670.03 & $a^{3} \mathrm{P}_{1}-y^{3} \mathrm{D}_{2}^{\circ}$ & $\left.\begin{array}{c}(0.00,0.33) \\
1.49\end{array}\right) .83,1.16$ & $\begin{array}{l}(0.00,0.32) \\
1.51\end{array}$ \\
\hline $\begin{array}{l}05.78 \\
01.65\end{array}$ & $\begin{array}{r}100 \\
3\end{array}$ & V E & $\begin{array}{l}705.79 \\
725.27\end{array}$ & $\begin{array}{l}a^{3} \mathrm{P}_{1}-z^{1} \mathrm{P}_{\mathrm{i}}^{\circ} \\
e^{3} \mathrm{G}_{5}-4_{4}^{\circ}\end{array}$ & $(0.63) 0.89,1.52$ & $(0.62) 0.88,1.51$ \\
\hline $4,600.59$ & $5 \mathrm{~h}$ & & 730.27 & $\begin{array}{l}y^{3} \mathrm{P}_{2}^{\circ}-f^{3} \mathrm{D}_{3} \\
y^{3} \mathrm{P}_{1}^{\circ}-f^{3} \mathrm{D}_{2}\end{array}$ & & \\
\hline $\begin{array}{r}4,595.06 \\
87.14 \\
80.05 \\
74.87\end{array}$ & $\begin{aligned} 2 \mathrm{~h} \\
2 \mathrm{~h} \\
150 \\
200\end{aligned}$ & III $\underset{E}{E}$ & $\begin{array}{l}756.42 \\
793.99 \\
827.73 \\
852.44\end{array}$ & $\begin{array}{l}e^{3} \mathrm{~F}_{3}-2 \stackrel{\circ}{4} \\
a^{3} \mathrm{P}_{1}-y^{3} \mathrm{P}_{0}^{\circ} \\
a^{1} \mathrm{D}_{2}-2^{3} \mathrm{P}_{2}^{\circ}\end{array}$ & $\begin{array}{l}(0.00) 1.51 \\
(0.49,0.98) 0.49,0.98 \\
\quad 1.46,1.94\end{array}$ & $\begin{array}{l}(0.00) 1.51 \\
(0.48,0.95) \\
\quad 1.46,1.94\end{array}$ \\
\hline $\begin{array}{l}70.97 \\
62.5\end{array}$ & $\begin{array}{l}10 \\
5 \mathrm{~h}\end{array}$ & V E & $\begin{array}{l}871.08 \\
911.7\end{array}$ & $\begin{array}{l}a^{3} \mathrm{D}_{2}-y^{1} \mathrm{D}_{2} \\
e^{3} \mathrm{G}_{3}-1_{3}^{\circ}\end{array}$ & $(0.43) 1.00 \mathrm{R}$ & $(0.44) 1.02$ \\
\hline 59.28 & 100 & V E & 927.16 & $a^{3} \mathrm{P}_{2}-y^{3} \mathrm{P}_{1}^{\circ}$ & $(0.00,0.18) 1.62$ & $\begin{array}{c}(0.00,0.23) \\
1.71\end{array}$ \\
\hline $\begin{array}{l}58.46 \\
40.71 \\
38.87 \\
30.54\end{array}$ & $\begin{array}{l}200 \\
10 \\
8 \mathrm{hl} \\
15\end{array}$ & $\begin{array}{l}\text { III E } \\
\text { V E }\end{array}$ & $\begin{array}{r}21,931.10 \\
22,016.84 \\
025.76 \\
066.26\end{array}$ & $\begin{array}{l}a^{3} \mathrm{D}_{2}-y^{1} \mathrm{~F}_{3}^{\circ} \\
2^{1} \mathrm{~F}_{3}^{\circ}-e^{3} \mathrm{~F}_{4} \\
y^{3} \mathrm{P}_{2}^{\circ}-f^{1} \mathrm{D}_{2} \\
b^{1} \mathrm{D}_{2}-x^{3} \mathrm{P}_{1}^{\circ}\end{array}$ & $\begin{array}{l}(0.00 \mathrm{w}) 0.88 \mathrm{~A}^{1} \\
(0.00) 1.27\end{array}$ & $\begin{array}{l}(0.00) 0.82 \mathrm{~A}^{1} \\
(0.00) 1.28\end{array}$ \\
\hline $\begin{array}{l}26.12 \\
25.31\end{array}$ & $\begin{array}{l}200 \\
100\end{array}$ & III $\underset{\mathrm{V}}{\mathrm{E}}$ & $\begin{array}{l}087.81 \\
091.76\end{array}$ & $\begin{array}{l}a^{3} \mathrm{P}_{2}-y^{3} \mathrm{D}_{3}^{\circ} \\
z^{3} \mathrm{~F}_{4}^{\circ}-e^{3} \mathrm{~F}_{4}\end{array}$ & $\begin{array}{l}(0.00 \mathrm{~W}) 1.01 \mathrm{~A}^{1} \\
(0.46) 1.19 \mathrm{~B}\end{array}$ & $\begin{array}{l}(0.00) 0.96 \mathrm{~A}^{1} \\
(0.39) 1.20\end{array}$ \\
\hline 22.37 & 400 & III $\mathrm{E}$ & 106.12\{ & $\left.\begin{array}{r}b^{1} \mathrm{D}_{2}-x^{1} \mathrm{~F}_{3}^{\circ} \\
a^{3} \mathrm{~F}_{2}-2^{3} \mathrm{D}_{2}^{\circ}\end{array}\right\}$ & $(0.00) 1.02$ & $(0.00) 1.00$ \\
\hline $\begin{array}{l}16.38 \\
08.48\end{array}$ & $\begin{array}{c}5 \mathrm{hl} \\
10\end{array}$ & & $\begin{array}{l}135.44 \\
174.22\end{array}$ & $a^{3} \mathrm{P}_{0}-z^{1} \mathrm{P}_{1}^{0}$ & $(0.00) 0.90$ & $(0.00)^{-0.88}$ \\
\hline
\end{tabular}


TABLE 4.-The first spark spectrum of lanthanum (La II)-Continued

\begin{tabular}{|c|c|c|c|c|c|c|}
\hline \multirow{2}{*}{$\lambda_{\Delta \text { ir }}$ I. $A}$. & \multirow{2}{*}{$\begin{array}{l}\text { Inten- } \\
\text { sity } \\
\text { spark }\end{array}$} & \multirow{2}{*}{$\begin{array}{l}\text { Temper- } \\
\text { ature } \\
\text { class }\end{array}$} & \multirow{2}{*}{$\nu_{\mathrm{VBC}} \mathrm{cm}^{-1}$} & \multirow{2}{*}{$\begin{array}{l}\text { Term combi- } \\
\text { nations }\end{array}$} & \multicolumn{2}{|c|}{ Zeeman effects } \\
\hline & & & & & Observed & Computed \\
\hline $\begin{array}{r}05.82 \\
4,502.16 \\
4,498.76 \\
97.00 \\
84.48\end{array}$ & $\begin{array}{l}3 \mathrm{hl} \\
10 \mathrm{hl} \\
10 \\
2 \\
1 \mathrm{~h}\end{array}$ & t & $\begin{array}{l}187.32 \\
205.35 \\
222.13 \\
230.83 \\
292.90\end{array}$ & $\begin{array}{l}y^{3} \mathrm{P}_{0}^{0}-f^{3} \mathrm{D}_{1} \\
x^{3} \mathrm{P}_{1}^{\circ}-e^{3} \mathrm{~S}_{1} \\
y^{3} \mathrm{~F}_{3}^{0}-e^{1} \mathrm{D}_{2} \\
a^{3} \mathrm{~F}_{3}-z^{3} \mathrm{P}_{2}^{\circ} \\
e^{1} \mathrm{G}_{4}-6_{4,3}^{\circ}\end{array}$ & $\begin{array}{l}(0.00) 1.47 \\
(0.00) 1.14\end{array}$ & $\begin{array}{l}(0.00) 1.47 \\
(0.00) 1.13\end{array}$ \\
\hline $\begin{array}{l}81.21 \\
74.03\end{array}$ & $\begin{array}{l}25 \mathrm{hl} \\
10\end{array}$ & & $\begin{array}{l}309.16 \\
344.96\end{array}$ & $\begin{aligned} z^{1} \mathrm{P}_{1}^{\circ}-\gamma^{3} \mathrm{D}_{1} \\
y^{3} \mathrm{D}_{2}^{0}-\gamma^{3} \mathrm{D}_{1}\end{aligned}$ & $\begin{array}{l}(0.40) 0.50,0.87 \\
(0.00,0.66) 0.50,1.17 \\
1.83\end{array}$ & 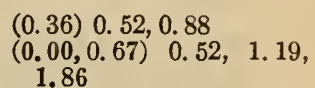 \\
\hline $\begin{array}{l}59.10 \\
55.79 \\
43.94\end{array}$ & $\begin{array}{l}3 \\
50 \\
20 \mathrm{hl}\end{array}$ & V E & $\begin{array}{l}419.78 \\
436.43 \\
496.26\end{array}$ & $\begin{array}{l}z^{1} \mathrm{G}_{4}^{0}-e^{3} \mathrm{G}_{5} \\
a^{3} \mathrm{P}_{1}-y^{3} \mathrm{P}_{1}^{0} \\
y^{3} \mathrm{D}_{2}^{0}-f^{3} \mathrm{D}_{2}\end{array}$ & $\begin{array}{l}(0.24) 1.41 \\
(0.00) 1.18 \mathrm{~h}\end{array}$ & $\begin{array}{l}(0.24) 1.38 \\
(0.09) \\
(.17\end{array}$ \\
\hline 35.84 & 10 & IV $\mathrm{F}$ & 537.34 & $a^{3} \mathrm{~F}_{2}^{\circ}-z^{3} \mathrm{D}_{3}^{\circ}$ & $\begin{array}{l}(0.00,0.60,1.19) 1.28, \\
1.86,2.44\end{array}$ & $\begin{array}{l}(0.00,0.59,1.17)-,-, \\
\quad 1.32,1.90,2.49\end{array}$ \\
\hline $\begin{array}{l}32.95 \\
29.90\end{array}$ & $\begin{array}{c}20 ? \\
400\end{array}$ & III E & $\begin{array}{l}552.03 \\
567.56\end{array}$ & $\begin{array}{l}a^{3} \mathrm{~F}_{4}-y^{1} \mathrm{~F}_{3}^{\circ} \\
a^{3} \mathrm{D}_{1}-y^{1} \mathrm{D}_{2}^{\circ}\end{array}$ & $(0.00,0.40) 0.51,0.91$, & $(0.00,0.38) \quad 0.52,0.90$, \\
\hline $\begin{array}{l}27.52 \\
19.16\end{array}$ & $\begin{array}{r}100 \\
30\end{array}$ & $\begin{array}{l}\mathrm{V} \\
\mathrm{V}\end{array}$ & $\begin{array}{l}579.69 \\
622.41\end{array}$ & $\begin{array}{l}z^{3} \mathrm{~F}_{3}^{\circ}-e^{3} \mathrm{~F}_{3} \\
2^{1} \mathrm{G}_{4}^{0}-e^{1} \mathrm{G}_{4}\end{array}$ & $\begin{array}{l}(0.00 \mathrm{w}) 1.06 \\
(0.28) 1.04\end{array}$ & $\begin{array}{l}(0.07) 1.07 \\
(0.21) 1.03\end{array}$ \\
\hline $\begin{array}{r}17.14 \\
12.22 \\
11.21 \\
4,403.02 \\
4,385.20\end{array}$ & $\begin{array}{l}2 \mathrm{~h} \\
2 \mathrm{~h} \\
25 \mathrm{hl} \\
2 \\
40\end{array}$ & $\begin{array}{l}\text { III } \\
\text { V E } \\
\text { III } \\
\text { V }\end{array}$ & $\begin{array}{l}632.75 \\
657.98 \\
663.17 \\
705.33 \\
797.59\end{array}$ & $\begin{array}{l}x^{3} \mathrm{P}_{1}^{\circ}-e^{1} \mathrm{~S}_{0} \\
e^{3} \mathrm{~F}_{3}-3_{3}^{\circ}, 2 \\
x^{3} \mathrm{~F}_{4}^{\circ}-f^{3} \mathrm{D}_{3} \\
a^{3} \mathrm{~F}_{2}-z^{3} \mathrm{P}_{1}^{\circ} \\
z^{3} \mathrm{~F}_{33}^{\circ}-e^{3} \mathrm{G}_{4}\end{array}$ & $\begin{array}{l}(0.00 \mathrm{~h}) 1.13 \mathrm{~h} \\
(0.00) 1.24\end{array}$ & $\begin{array}{l}(0.00) 1.12 \\
(0.00) 1.24\end{array}$ \\
\hline 83.44 & 100 & V E & 806.75 & $z^{3} \mathrm{~F}_{2}^{\circ}-e^{3} \mathrm{~F}_{3}$ & $(0.00,0.40,0.80) \quad 0.28$, & $(0.00,0.38,0.77) \quad 0.29$, \\
\hline $\begin{array}{l}78.10 \\
64.66 \\
63.05 \\
56.18\end{array}$ & $\begin{array}{c}50 \\
100 \\
501 \\
1\end{array}$ & $\begin{array}{l}\text { IV } \\
\text { IV } \\
\text { V } \\
\mathrm{E}\end{array}$ & $\begin{array}{l}834.56 \\
904.88 \\
913.33 \\
949.46\end{array}$ & $\begin{array}{l}z^{3} \mathrm{~F}_{3}^{\circ}-e^{1} \mathrm{~F}_{3} \\
a^{3} \mathrm{P}_{0}-y^{3} \mathrm{P}_{1}^{\circ} \\
y^{3} \mathrm{D}_{3}^{\circ}--^{3} \mathrm{D}_{3} \\
y^{1} \mathrm{P}_{1}^{\circ}-e^{1} \mathrm{P}_{1}\end{array}$ & $\begin{array}{l}(0.30 \mathrm{~W}) 0.95 \\
(0.00) 1.28 \\
(0.00 \mathrm{~h}) 1.32 \mathrm{~h}\end{array}$ & $\begin{array}{l}(0.34) 1.02 \\
(0.00) 1.26 \\
(0.03) 1.32\end{array}$ \\
\hline $\begin{array}{l}54.40 \\
37.78 \\
34.96\end{array}$ & $\begin{array}{l}200 \\
101 \\
100\end{array}$ & $\begin{array}{l}\text { IV E } \\
\text { V E }\end{array}$ & $\begin{array}{r}22,958.84 \\
23,046.81 \\
061.80\end{array}$ & $\begin{array}{l}a^{1} \mathrm{~S}_{0}-y^{1} \mathrm{P}_{1}^{\circ} \\
x^{3} \mathrm{~F}_{3}^{\circ}-f^{3} \mathrm{D}_{2} \\
z^{3} \mathrm{~F}_{2}^{0}-e^{1} \mathrm{~F}_{3}\end{array}$ & $\begin{array}{l}(0.00) 1.08 \\
(0.00) 1.08 \mathrm{~h} \\
(0.00,0.29,0.57) \\
0.97,1.26,1.54\end{array}$ & $\begin{array}{l}(0.00) 1.07 \\
(0.00) 1.02 \\
(0.00,0.28,0.55)-0.68, \\
0.95,1.23,1.51\end{array}$ \\
\hline $\begin{array}{l}33.76 \\
22.51\end{array}$ & $\begin{array}{l}500 \\
100\end{array}$ & $\begin{array}{l}\text { III E } \\
\text { III E }\end{array}$ & $\begin{array}{l}068.19 \\
128.22\end{array}$ & $\begin{array}{l}a^{1} \mathrm{D}_{2}-y^{1} \mathrm{D}_{2}^{\circ} \\
a^{1} \mathrm{D}_{2}-y^{1} \mathrm{~F}_{3}^{\circ}\end{array}$ & $\begin{array}{l}(0.17) 0.94 \\
(0.00) 1.10\end{array}$ & $\begin{array}{l}(0.16) 0.94 \\
(0.00) 1.08\end{array}$ \\
\hline 15.90 & 30 & V E & 163.65 & $a^{3} D_{3}-x^{3} F_{2}^{\circ}$ & $\begin{array}{c}(0.00,0.50,1.00) \\
1.36,1.86,2.36\end{array}$ & $\begin{array}{l}(0.00,0.50,1.00)-0.84, \\
\quad 1.34,1.84,2.34\end{array}$ \\
\hline $\begin{array}{r}04.11 \\
4,300.44\end{array}$ & $\begin{array}{l}10 \mathrm{hl} \\
60\end{array}$ & IV E & $\begin{array}{l}227.10 \\
246.92\end{array}$ & $\begin{array}{l}e^{3} \mathrm{~F}_{4}-5_{4,3}^{\circ}, 3 \\
a^{3} \mathrm{~F}_{2}-z^{3} \mathrm{P}_{2}^{\circ}\end{array}$ & $\begin{array}{l}(0.00 \mathrm{~h}) 1.24 \mathrm{~h} \\
(0.73,1.48) \quad 0.00,0.72 \text {, }\end{array}$ & $(0.73,1.46) 0.00,0.73$ \\
\hline $\begin{array}{r}4,296.05 \\
86.97\end{array}$ & $\begin{array}{l}300 \\
300\end{array}$ & IV $\underset{V}{\mathrm{E}}$ & $\begin{array}{l}270.67 \\
319.96\end{array}$ & $\begin{array}{l}a^{3} \mathrm{P}_{2}-y^{3} \mathrm{P}_{2}^{0} \\
2^{3} \mathrm{~F}_{1}^{0}-e^{3} \mathrm{G}_{5}\end{array}$ & $\begin{array}{l}(0.00) 1.48 \\
(0.00) 1.08\end{array}$ & $\begin{array}{l}(0.02) 1.49 \\
(0.00) \\
(.09\end{array}$ \\
\hline 75.64 & 100 & IV E & 381.75 & $a^{3} \mathrm{D}_{2}-y^{3} \mathrm{D}_{i}^{\circ}$ & $(0.00,0.35) 0.82,1.17$ & $(0.00,0.34) \quad 0.80,1.14$ \\
\hline $\begin{array}{l}69.50 \\
63.59 \\
59.51 \\
56.50\end{array}$ & $\begin{array}{l}300 \\
200 \\
2 \mathrm{~h} \\
3\end{array}$ & $\begin{array}{l}\mathrm{V} \\
\mathrm{V}\end{array}$ & $\begin{array}{l}415.38 \\
447.83 \\
470.30 \\
486.89\end{array}$ & $\begin{array}{l}z^{3} \mathrm{~F}_{3}^{0}-e^{3} \mathrm{~F}_{4} \\
z^{1} \mathrm{~F}_{0}^{0}-e^{1} \mathrm{G}_{4} \\
x^{3} \mathrm{~F}_{2}^{2}-f^{3} \mathrm{D}_{2}\end{array}$ & $\begin{array}{ll}(0.00) & 1.20 \\
(0.00) & 1.12\end{array}$ & $\begin{array}{l}(0.00) 1.22 \\
(0.00) \\
1.08\end{array}$ \\
\hline $\begin{array}{l}52.93 \\
49.99\end{array}$ & $\begin{array}{r}4 \\
100\end{array}$ & V E & $\begin{array}{l}506.61 \\
522.87\end{array}$ & $\begin{array}{l}a^{3} \mathrm{~F}_{3}-y^{1} \mathrm{~F}_{3}^{\circ} \\
z^{3} \mathrm{~F}_{4}^{\circ}-e^{1} \mathrm{G}_{4}\end{array}$ & $\begin{array}{l}(0.74) 0.50,0.76,1.02, \\
\quad 1.27,1.53,1.76\end{array}$ & $\begin{array}{l}(-,-, 0.59,0.79) \\
0.75,1.00,1.25,1.50 \\
1.75\end{array}$ \\
\hline $\begin{array}{l}48.32 \\
41.20 \\
38.38\end{array}$ & $\begin{array}{l}2 \\
15 \mathrm{hl} \\
400\end{array}$ & III $\mathrm{E}$ & $\begin{array}{l}532.11 \\
571.62 \\
587.30\end{array}$ & $\begin{array}{l}y^{1} \mathrm{P}_{1}^{\circ}-f^{3} \mathrm{~F}_{2} \\
e^{3} \mathrm{G}_{4}-4_{4}^{\circ} \\
a^{3} \mathrm{D}_{3}-x^{3} \mathrm{~F}_{3}^{\circ}\end{array}$ & $\begin{array}{l}(0.74) 0.56,0.82,1.08 \\
\quad 1.34,1.60,1.86\end{array}$ & $\begin{array}{r}(-, 0.51,0.77) 0.57,0.83, \\
1.08,1.34,1.59,1.85\end{array}$ \\
\hline 30.95 & 150 & V E & 628.72 & $z^{1} \mathrm{~F}_{3}^{\circ}-e^{3} \mathrm{D}_{3}$ & $\begin{array}{l}(0.80) 0.65,0.85,1.06 \\
1.26,1.47,1.67 \mathrm{ur}\end{array}$ & $\begin{array}{r}(-, 0.52,0.78) \\
1.05,1.31,1.56,1.89\end{array}$ \\
\hline $\begin{array}{l}17.56 \\
10.22 \\
07.61 \\
04.03\end{array}$ & $\begin{array}{l}200 \\
50 \mathrm{hl} \\
10 \mathrm{l} \\
100\end{array}$ & $\mathrm{VE}$ & $\begin{array}{l}703.74 \\
745.06 \\
759.79 \\
780.02\end{array}$ & $\begin{array}{l}2^{3} \mathrm{~F}_{4}^{\circ}-e^{3} \mathrm{D}_{3} \\
y^{3} \mathrm{D}_{1}^{\circ}-f^{3} \mathrm{D}_{1} \\
a^{3} \mathrm{P}_{1}-y^{3} \mathrm{P}_{2}^{\circ}\end{array}$ & $\begin{array}{l}(0.00) 1.22 \\
(0.00 \mathrm{~h}) 1.02 \mathrm{~h} \\
(0.27) 0.68 \mathrm{~h} \\
(0.00) 1.48\end{array}$ & $\begin{array}{l}(0.00) 1.19 \\
(0.28) 0.66 \\
(0.00) 1.49\end{array}$ \\
\hline $\begin{array}{l}4,201.50 \\
4,196.55\end{array}$ & $\begin{array}{l}6 \mathrm{~h} \\
250\end{array}$ & III E & $\begin{array}{l}794.34 \\
822.41\end{array}$ & $a^{3} \mathrm{D}_{2}-x^{3} \mathrm{~F}_{2}$ & $\begin{array}{l}(0.32,0.64) 0.51,0.82, \\
1.13,1.44\end{array}$ & $\begin{array}{c}(0.30,0.61) 0.53,0.84 \text {, } \\
1.14,1.44\end{array}$ \\
\hline $\begin{array}{l}94.36 \\
93.34 \\
92.35\end{array}$ & $\begin{array}{c}30 \mathrm{~h} \\
5 \\
100\end{array}$ & V E & $\begin{array}{l}834.85 \\
840.64 \\
846.27\end{array}$ & $\begin{array}{r}x^{1} \mathrm{~F}_{3}^{\circ}-f^{1} \mathrm{G}_{4} \\
{\left[y^{3} \mathrm{D}_{2}^{\circ}-f^{3} \mathrm{D}_{3}\right]} \\
z^{3} \mathrm{~F}_{3}^{\circ}-e^{3} \mathrm{D}_{2}\end{array}$ & $\begin{array}{l}(0.00 \mathrm{~h}) 1.06 \mathrm{~h} \\
(0.25) 0.64 \\
(0.00) 1.06\end{array}$ & $\begin{array}{ll}(0.00) & 1.06 \\
(0.00) & 1.47(?) \\
(0.00) & 1.07\end{array}$ \\
\hline
\end{tabular}


TABLE 4.-The first spark spectrum of lanthanum ( $L a$ II)-Continued

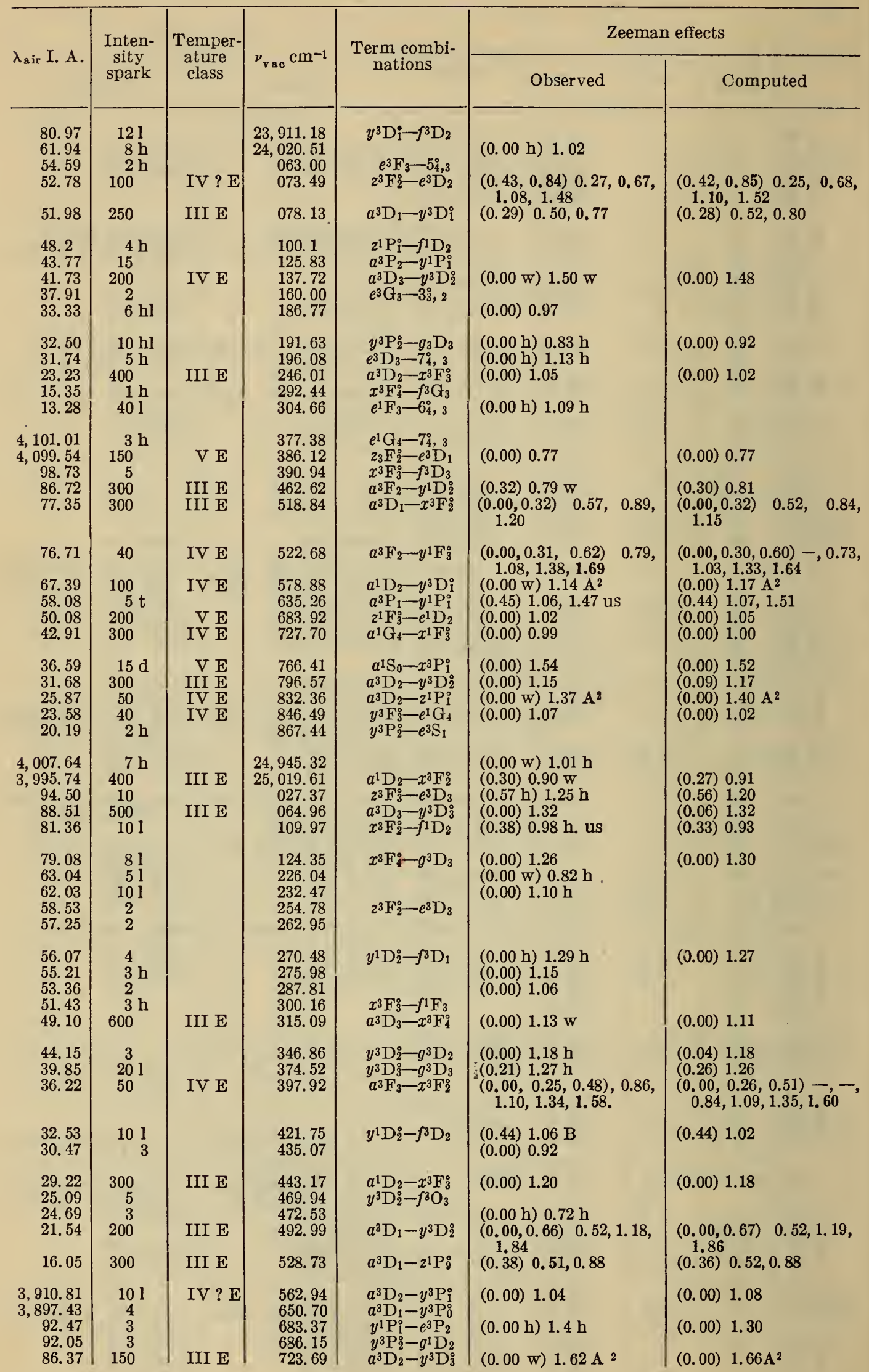


TABLE 4.-The first spark spectrum of lanthanum (La II)-Continued

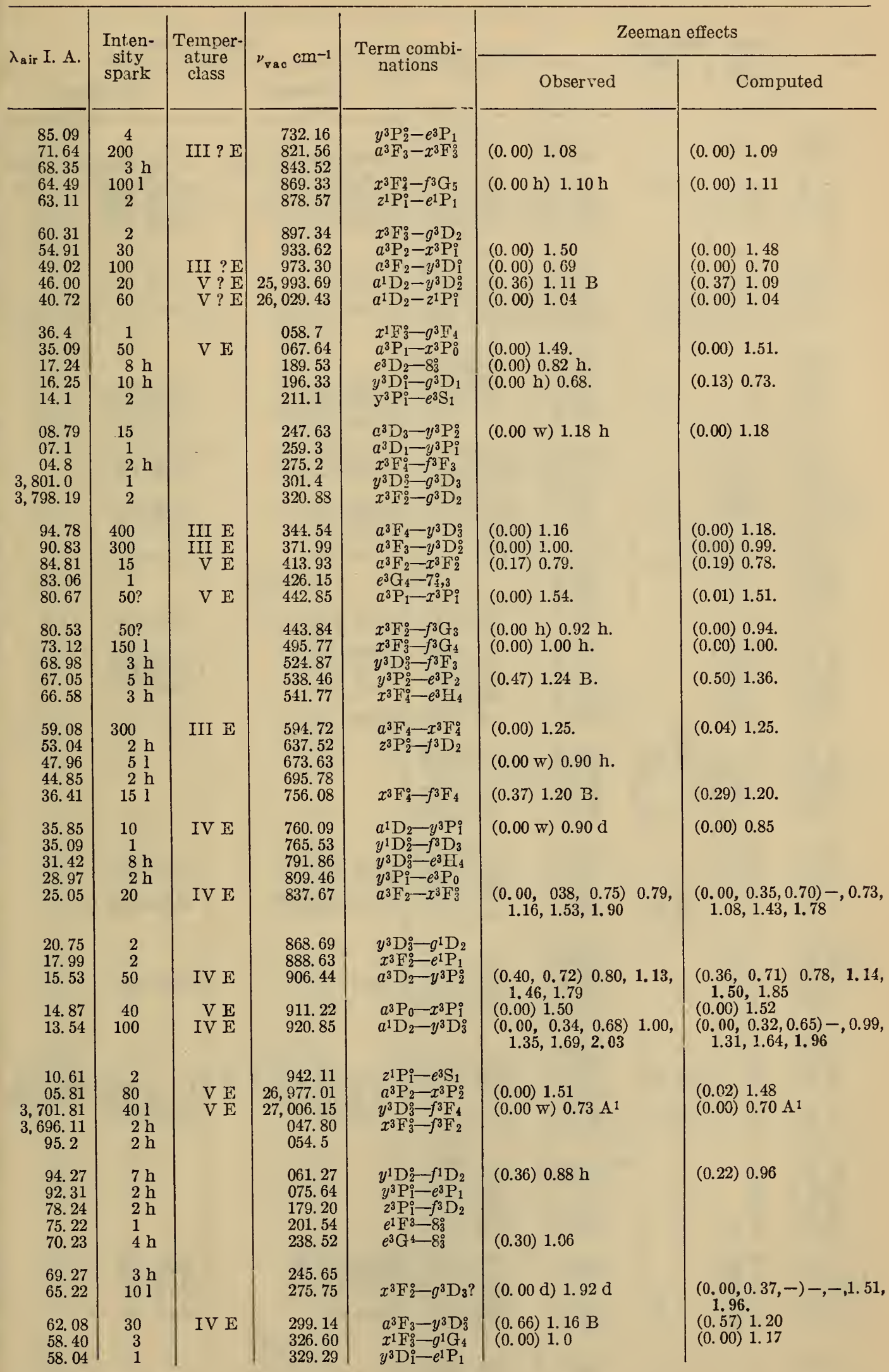


TABLE 4.-The first spark spectrum of lanthanum (La II)-Continued

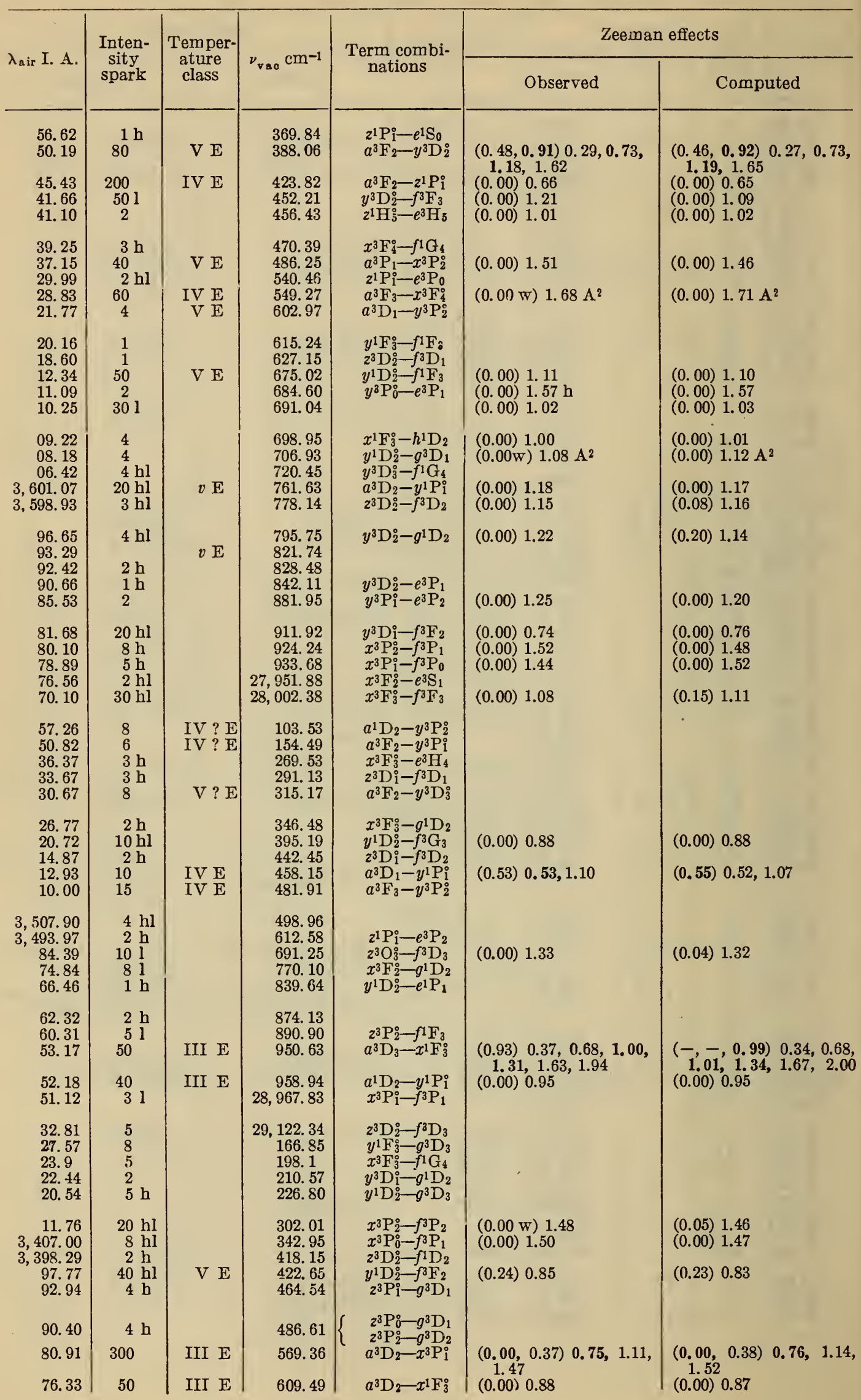


TABLE 4.-The first spark spectrum of lanthanum (La II)-Continued

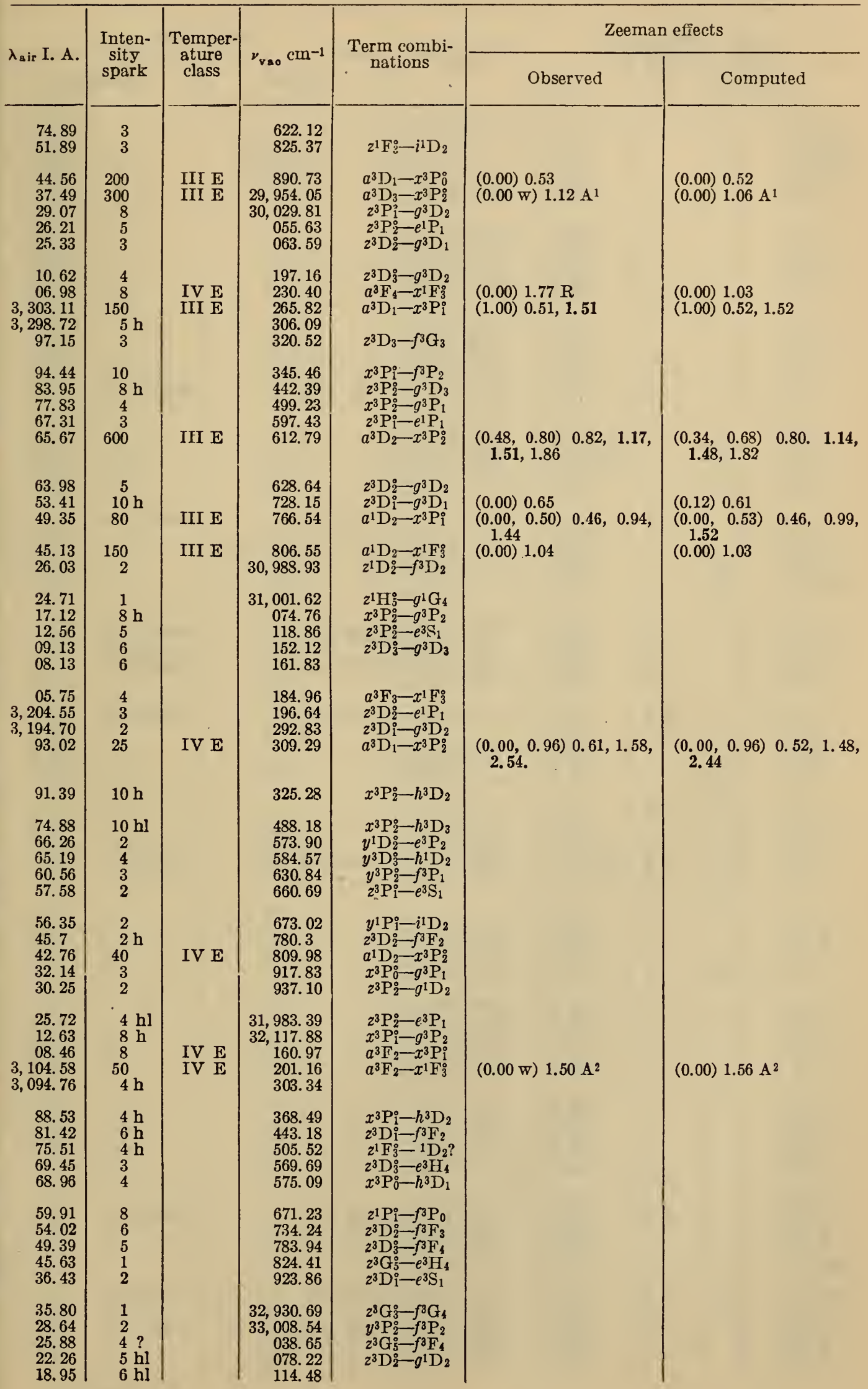


- TABLE 4.-The first spark spectrum of lanthanum (La II)-Continued

\begin{tabular}{|c|c|c|c|c|c|c|c|c|}
\hline \multirow{2}{*}{$\lambda_{\mathrm{a} \text { ir }}$ I. A. } & \multirow{2}{*}{$\begin{array}{l}\text { Inten- } \\
\text { sity } \\
\text { spark }\end{array}$} & \multirow{2}{*}{$\begin{array}{c}\text { Temper- } \\
\text { ature } \\
\text { class }\end{array}$} & \multirow{2}{*}{$\nu_{\text {Vac }} \mathrm{cm}^{-1}$} & \multirow{2}{*}{$\begin{array}{l}\text { Term combi- } \\
\text { nations }\end{array}$} & \multicolumn{4}{|c|}{ Zeeman effects } \\
\hline & & & & & & Observed & & Computed \\
\hline $\begin{array}{r}07.32 \\
3,004.68 \\
2,985.76 \\
85.43 \\
84.33\end{array}$ & $\begin{array}{l}5 \\
5 \mathrm{~h} \\
2 \\
5 \\
3\end{array}$ & & $\begin{array}{l}242.54 \\
271.74 \\
482.57 \\
486.27 \\
498.61\end{array}$ & $\begin{array}{l}z^{1} \mathrm{D}_{2}^{\circ}-f^{1} \mathrm{~F}_{3} \\
z^{3} \mathrm{G}_{3}^{\circ}-f^{3} \mathrm{~F}_{2} \\
x^{3} \mathrm{~F}_{2}^{\circ}-h^{1} \mathrm{D}_{2} \\
z^{3} \mathrm{D}_{3}^{\circ}-f^{1} \mathrm{G}_{4}\end{array}$ & & & & \\
\hline $\begin{array}{l}83.44 \\
76.83 \\
71.48 \\
66.55 \\
66.08\end{array}$ & $\begin{array}{l}3 \\
3 \\
1 \\
4 \\
2\end{array}$ & & $\begin{array}{l}508.61 \\
583.01 \\
643.47 \\
699.38 \\
704.72\end{array}$ & $\begin{array}{l}z^{3} \mathrm{G}_{4}^{0}-f^{3} \mathrm{~F}_{3} \\
y^{3} \mathrm{P}_{0}^{0}-f^{3} \mathrm{P}_{1} \\
y^{3} \mathrm{~F}^{0}-f^{3} \mathrm{G}_{3} \\
z^{3} \mathrm{G}_{5}^{\circ}-e^{3} \mathrm{H}_{5} \\
z^{1} \mathrm{P}_{1}^{\mathrm{i}}-f^{3} \mathrm{P}_{1}\end{array}$ & & & & \\
\hline $\begin{array}{l}62.90 \\
59.85 \\
58.71 \\
51.46 \\
50.50\end{array}$ & $\begin{array}{r}15 \\
5 \\
1 \\
3 \\
50\end{array}$ & $\mathrm{VE}$ & $\begin{array}{l}740.89 \\
775.65 \\
788.67 \\
871.66 \\
882.68\end{array}$ & $\begin{array}{l}y^{3} \mathrm{O}_{2}^{\circ}-f^{3} \mathrm{P}_{1} \\
z^{3} \mathrm{G}_{1}^{\circ}-e^{3} \mathrm{H}_{4} \\
z^{3} \mathrm{D}_{1}^{\circ}-e^{3} \mathrm{P}_{1} \\
y^{3} \mathrm{P}_{1}^{\circ}-i 1^{1} \mathrm{D}_{2} \\
z^{1} \mathrm{H}_{0}^{\circ}-e^{1} \mathrm{I}_{6}\end{array}$ & $(0.00)$ & 1.04 & $(0.00)$ & 1.04 \\
\hline $\begin{array}{l}48.82 \\
43.56 \\
39.64 \\
29.86 \\
25.15\end{array}$ & $\begin{array}{l}1 \\
6 \mathrm{hl} \\
3 \mathrm{~h} \\
7 \\
5 \mathrm{~h}\end{array}$ & & $\begin{array}{r}901.99 \\
33,962.57 \\
34,007.85 \\
121.37 \\
176.31\end{array}$ & $\begin{array}{c}y^{3} \mathrm{~F}_{3}^{\circ}-f^{1} \mathrm{~F}_{3} \\
z^{1} \mathrm{D}_{2}^{\circ}-f^{3} \mathrm{G}_{3} \\
y^{1} \mathrm{P}_{1}^{\circ}-h^{3} \mathrm{D}_{1} \\
y^{3} \mathrm{D}_{1}^{\circ}-f^{3} \mathrm{P}_{0} \\
y^{1} \mathrm{P}_{1}^{\circ}-h^{3} \mathrm{D}_{2}\end{array}$ & & & & \\
\hline $\begin{array}{r}23.90 \\
13.60 \\
10.05 \\
2,905.53 \\
2,899.80\end{array}$ & $\begin{array}{l}20 \\
2 \\
1 \\
4 \mathrm{hl} \\
4 \mathrm{hl}\end{array}$ & & $\begin{array}{l}190.92 \\
311.78 \\
353.63 \\
407.07 \\
475.06\end{array}$ & $\begin{array}{l}y^{3} \mathrm{D}_{3}^{\circ}-f^{3} \mathrm{P}_{2} \\
y^{3} \mathrm{~F}_{2}^{\circ}-f^{1} \mathrm{D}_{2} \\
y^{1} \mathrm{P}_{1}^{\circ}-1 \mathrm{D}_{2} ? \\
z^{1} \mathrm{D}_{2}^{\circ}-e^{1} \mathrm{P}_{1} \\
y^{3} \mathrm{~F}_{4}^{\circ}-g^{3} \mathrm{D}_{3}\end{array}$ & $(0.00)$ & $1.23 ?$ & $(0.00)$ & 1.17 \\
\hline $\begin{array}{l}97.76 \\
93.08 \\
89.11 \\
85.13 \\
83.35\end{array}$ & $\begin{array}{l}5 \mathrm{hl} \\
60 \\
1 \\
50 \\
1\end{array}$ & $\begin{array}{l}\mathrm{VE} \\
\mathrm{VE}\end{array}$ & $\begin{array}{l}499.32 \\
555.14 \\
602.61 \\
650.35 \\
671.74\end{array}$ & $\begin{array}{l}y^{3} \mathrm{~F}_{3}^{\circ}-g^{3} \mathrm{D}_{2} \\
z^{3} \mathrm{G}_{5}^{\circ}-e^{3} \mathrm{H}_{6} \\
z^{1} \mathrm{P}_{1}^{\circ}-i^{1} \mathrm{D}_{2} \\
z^{3} \mathrm{G}_{4}^{\circ}-e^{3} \mathrm{H}_{5} \\
z^{3} \mathrm{P}_{2}^{\circ}-g^{3} \mathrm{~F}_{3}\end{array}$ & $\begin{array}{l}(0.00) \\
(0.00)\end{array}$ & $\begin{array}{l}1.12 \\
1.04\end{array}$ & $\begin{array}{l}(0.00) \\
(0.00)\end{array}$ & $\begin{array}{l}1.12 \\
1.00\end{array}$ \\
\hline $\begin{array}{l}80.65 \\
76.55 \\
74.28 \\
73.20 \\
67.47\end{array}$ & $\begin{array}{l}40 \\
1 \\
3 \\
2 \\
2 \mathrm{~h}\end{array}$ & $\mathrm{VE}$ & $\begin{array}{l}704.23 \\
753.69 \\
781.14 \\
794.21 \\
863.74\end{array}$ & $\begin{array}{l}z^{3} \mathrm{G}_{3}^{\circ}-e^{3} \mathrm{H}_{4} \\
z^{3} \mathrm{G}_{3}^{\circ}-g^{1} \mathrm{D}_{2} \\
z^{1} \mathrm{D}_{2}^{\circ}-g^{3} \mathrm{D}_{3} \\
y^{3} \mathrm{P}_{2}^{0}-h^{3} \mathrm{D}_{1}\end{array}$ & $(0.00)$ & 1.18 & $(0.00)$ & 1.19 \\
\hline $\begin{array}{l}62.98 \\
62.37 \\
59.76 \\
55.90 \\
53.72\end{array}$ & $\begin{array}{l}15 \mathrm{hl} \\
6 \\
5 \\
50 \mathrm{hl} \\
4 \mathrm{~h}\end{array}$ & $\begin{array}{l}\text { VE } \\
\text { V E }\end{array}$ & $\begin{array}{r}918.41 \\
925.85 \\
34,957.73 \\
35,004.97 \\
031.71\end{array}$ & $\begin{array}{l}z^{3} \mathrm{G}_{3}^{\circ}-f^{3} \mathrm{~F}_{4} \\
y^{3} \mathrm{~F}_{2}-f^{1} \mathrm{~F}_{3} \\
\left.y^{3} \mathrm{~F}_{2}^{\circ}-g^{3} \mathrm{~J}\right)_{1} \\
y^{1} \mathrm{~F}_{3}^{\circ}-g^{1} \mathrm{G}_{4} \\
y^{3} \mathrm{P}_{2}^{\circ}-h^{3} \mathrm{D}_{2}\end{array}$ & $(0.00)$ & 1.13 & $(0.00)$ & 1.13 \\
\hline $\begin{array}{l}49.51 \\
48.34 \\
46.67 \\
43.67 \\
40.51\end{array}$ & $\begin{array}{l}2 \mathrm{~h} \\
6 \\
5 \\
4 \\
25 \mathrm{hl}\end{array}$ & & $\begin{array}{l}083.47 \\
097.88 \\
118.47 \\
155.51 \\
194.62\end{array}$ & $\begin{array}{c}y^{3} \mathrm{~T}_{3}^{\circ}-f_{3} \mathrm{G}_{4} \\
y^{3} \mathrm{D}_{2}^{\circ}-f^{3} \mathrm{P}_{2} \\
y^{3} \mathrm{D}_{1}^{\circ}-f^{3} \mathrm{P}_{1} \\
y^{3} \mathrm{P}_{2}^{\circ}-h^{3} \mathrm{D}_{3}\end{array}$ & & & $f$ & \\
\hline $\begin{array}{l}38.45 \\
32.53 \\
25.82 \\
25.51 \\
21.03\end{array}$ & $\begin{array}{l}51 \\
5 \\
1 \\
5 \\
5\end{array}$ & & $\begin{array}{l}220.16 \\
293.77 \\
377.57 \\
381.45 \\
437.64\end{array}$ & $\begin{array}{c}y^{3} \mathrm{~F}_{4}^{\circ}-f^{3} \mathrm{G}_{5} \\
z^{3} \mathrm{D}_{2}^{\circ}-g^{3} \mathrm{~F}_{2} \\
y^{1} \mathrm{~F}_{3}^{\circ}-h^{1} \mathrm{D}_{2} \\
z^{3} \mathrm{D}_{3}^{3}-g^{3} \mathrm{~F}_{3} \\
y^{1} \mathrm{D}_{2}^{\circ}-h^{1} \mathrm{D}_{2}\end{array}$ & & & & \\
\hline $\begin{array}{l}19.73 \\
18.40 \\
15.36 \\
13.72 \\
13.05\end{array}$ & $\begin{array}{l}2 \\
3 \\
6 \\
5 \\
3\end{array}$ & & $\begin{array}{l}453.97 \\
470.70 \\
509.00 \\
529.70 \\
538.16\end{array}$ & $\begin{array}{l}y^{3} \mathrm{~F}_{3}^{\circ}-g^{3} \mathrm{D}_{3} \\
z^{1} \mathrm{D}_{2}^{\circ}-e^{3} \mathrm{~S}_{1} \\
z^{3} \mathrm{~F}_{3}^{\circ}-f^{3} \mathrm{D}_{2} \\
z^{3} \mathrm{~F}_{4}^{\circ}-f^{3} \mathrm{D}_{3} \\
z^{1} \mathrm{G}_{4}^{\circ}-f^{1} \mathrm{~F}_{3}\end{array}$ & & & & \\
\hline $\begin{array}{r}09.35 \\
08.39 \\
07.20 \\
05.58 \\
2,804.55\end{array}$ & $\begin{array}{r}2 \\
150 \\
1 \\
5 \\
4\end{array}$ & $\mathrm{VE}$ & $\begin{array}{l}584.96 \\
597.13 \\
612.22 \\
632.78 \\
645.86\end{array}$ & $\begin{array}{l}z^{3} \mathrm{~F}_{2}^{\circ}-f^{3} \mathrm{D}_{1} \\
b^{1} \mathrm{D}_{2}-x^{1} \mathrm{P}_{1}^{\circ} \\
x^{3} \mathrm{~F}_{2}^{\circ}-i^{1} \mathrm{D}_{2} \\
z^{3} \mathrm{G}_{3}^{\circ}-f^{1} \mathrm{G}_{4} \\
y^{3} \mathrm{~F}_{2}^{\circ}-f^{3} \mathrm{G}_{3}\end{array}$ & $(0.00)$ & 1.04 & $(0.00)$ & 1.04 \\
\hline $\begin{array}{r}2,798.56 \\
96.40 \\
91.51 \\
81.25 \\
80.23\end{array}$ & $\begin{array}{l}40 \mathrm{hl} \\
5 \\
25 \\
1 \\
20\end{array}$ & $\mathrm{VE}$ & $\begin{array}{l}722.15 \\
749.75 \\
812.37 \\
944.47 \\
957.66\end{array}$ & $\begin{array}{c}z^{3} \mathrm{D}_{3}^{\circ}-g^{3} \mathrm{~F}_{4} \\
z^{1} \mathrm{~F}_{3}^{\circ}-f^{1} \mathrm{D}_{2} \\
z^{3} \mathrm{D}_{2}^{0}-g^{3} \mathrm{~F}_{3} \\
z^{1} \mathrm{D}_{2}^{0}-f^{3} \mathrm{~F}_{3} \\
z^{3} \mathrm{D}_{1}^{\circ}-g^{3} \mathrm{~F}_{2}\end{array}$ & $\begin{array}{l}(0.00) \\
(0.00)\end{array}$ & $\begin{array}{l}1.08 \\
1.04 \mathrm{R}\end{array}$ & $\begin{array}{l}(0.00) \\
(0.00)\end{array}$ & $\begin{array}{l}1.08 \\
1.03\end{array}$ \\
\hline
\end{tabular}


TABLE 4.-The first spark spectrum of lanthanum (La II)-Continued

\begin{tabular}{|c|c|c|c|c|c|c|}
\hline \multirow{2}{*}{$\lambda_{\mathrm{air}}$ I. A. } & \multirow{2}{*}{$\begin{array}{l}\text { Inten- } \\
\text { sity } \\
\text { spark }\end{array}$} & \multirow{2}{*}{$\begin{array}{c}\text { Temper- } \\
\text { ature } \\
\text { class }\end{array}$} & \multirow{2}{*}{$\nu_{\nabla \Delta 0} \mathrm{~cm}^{-1}$} & \multirow{2}{*}{$\begin{array}{l}\text { Term combi- } \\
\text { nations }\end{array}$} & \multicolumn{2}{|c|}{ Zeeman effects } \\
\hline & & & & & Observed & Computed \\
\hline $\begin{array}{l}79.78 \\
78.76 \\
73.86 \\
67.40 \\
61.10\end{array}$ & $\begin{array}{r}10 \\
10 \\
1 \\
8 \\
5\end{array}$ & & $\begin{array}{r}963.48 \\
35,976.68 \\
36,040.23 \\
124.35 \\
206.77\end{array}$ & $\begin{array}{c}y^{3} \mathrm{D}_{3}^{\circ}-g^{3} \mathrm{P}_{2} \\
2^{3} \mathrm{G}_{j}^{\circ}-g^{3} \mathrm{~F}_{4} \\
2^{1} \mathrm{P}_{1}^{\circ}-g^{3} \mathrm{P}_{0} \\
y^{3} \mathrm{P}_{1}^{\circ}-g^{3} \mathrm{P}_{2} \\
y^{3} \mathrm{P}_{1}^{\circ}-h^{3} \mathrm{D}_{1}\end{array}$ & . & \\
\hline $\begin{array}{l}60.51 \\
59.14 \\
58.65 \\
55.57 \\
52.84\end{array}$ & $\begin{array}{r}3 \\
3 \\
3 \\
1 \\
10\end{array}$ & & $\begin{array}{l}214.51 \\
232.49 \\
238.93 \\
279.43 \\
315.41\end{array}$ & $\begin{array}{l}y^{3} \mathrm{D}_{3}^{\circ}-h^{3} \mathrm{D}_{2} \\
z^{3} \mathrm{H}_{6}^{\circ}-e^{3} \mathrm{H}_{5} \\
y^{1} \mathrm{P}_{1}^{\circ}-f^{1} \mathrm{~S}_{0} \\
2^{1} \mathrm{P}_{1}^{\circ}-g^{3} \mathrm{P}_{1} \\
y^{3} \mathrm{D}_{2}^{\circ}-g^{3} \mathrm{P}_{1}\end{array}$ & & \\
\hline $\begin{array}{l}48.31 \\
36.90 \\
36.41 \\
32.40 \\
27.5\end{array}$ & $\begin{array}{r}8 \\
2 \\
3 \\
10 \\
2\end{array}$ & & $\begin{array}{l}375.26 \\
526.90 \\
533.44 \\
587.05 \\
6528\end{array}$ & $\begin{array}{l}y^{3} \mathrm{P}_{1}^{\circ}-h^{3} \mathrm{D}_{2} \\
z^{3} \mathrm{H}_{5}^{\circ}-e^{3} \mathrm{H}_{1} \\
y^{3} \mathrm{D}_{1}^{\circ}--^{3} \mathrm{P}_{2} \\
2^{3} \mathrm{G}_{4}^{\circ}-g^{3} \mathrm{~F}_{3} \\
z^{3} \mathrm{P}_{2}^{0}-h^{1} \mathrm{D}_{2}\end{array}$ & & \\
\hline $\begin{array}{l}26.48 \\
21.45 \\
15.43 \\
12.51 \\
09.92\end{array}$ & $\begin{array}{l}1 \\
2 \\
10 \mathrm{hl} \\
1 \\
3\end{array}$ & & $\begin{array}{l}666.49 \\
734.25 \\
815.68 \\
855.32 \\
890.54\end{array}$ & $\begin{array}{c}y^{1} \mathrm{D}_{2}^{\circ}-f^{3} \mathrm{P}_{1} \\
2^{1} \mathrm{G}_{4}^{0}-f^{3} \mathrm{G}_{4} \\
y^{3} \mathrm{P}_{0}^{0}-h^{3} \mathrm{D}_{1} \\
2^{1} \mathrm{P}_{1}^{\circ}-g^{3} \mathrm{P}_{2} \\
y^{3} \mathrm{D}_{2}^{\circ}-g^{3} \mathrm{P}_{2}\end{array}$ & f & \\
\hline $\begin{array}{r}06.49 \\
2,702.13 \\
2,695.47 \\
94.21 \\
91.60\end{array}$ & $\begin{array}{r}2 \\
8 \\
35 \\
5 \\
1\end{array}$ & & $\begin{array}{r}937.29 \\
36,996.88 \\
37,088.29 \\
105.64 \\
141.62\end{array}$ & $\begin{array}{c}z^{1} \mathrm{P}_{1}^{\circ}-h^{3} \mathrm{D}_{1} \\
z^{3} \mathrm{G}_{3}^{\circ}-g^{3} \mathrm{~F}_{2} \\
z^{3} \mathrm{H}_{6}^{\circ}-e^{3} \mathrm{H}_{6} \\
2^{1} \mathrm{P}_{1}^{\circ}-h^{3} \mathrm{D}_{2} \\
y^{3} \mathrm{D}_{2}^{\circ}-h^{3} \mathrm{D}_{2}\end{array}$ & & \\
\hline $\begin{array}{l}87.75 \\
81.49 \\
79.87 \\
75.66 \\
73.74\end{array}$ & $\begin{array}{c}2 \\
10 \\
4 \mathrm{~h} \\
5 \\
3\end{array}$ & & $\begin{array}{l}194.81 \\
281.64 \\
304.17 \\
362.87 \\
389.70\end{array}$ & $\begin{array}{l}2^{3} \mathrm{P}_{0}^{\circ}-h^{1} \mathrm{D}_{2} \\
2^{3} \mathrm{H}_{4}^{0}-e^{3} \mathrm{H}_{4} \\
y^{3} \mathrm{D}_{2}^{\circ}-h^{3} \mathrm{D}_{3} \\
z^{3} \mathrm{D}_{3}^{\circ}-h^{1} \mathrm{D}_{2} \\
2^{3} \mathrm{P}_{1}^{\circ}-f^{3} \mathrm{P}_{0}\end{array}$ & & \\
\hline $\begin{array}{l}72.90 \\
72.06 \\
70.05 \\
66.54 \\
66.18\end{array}$ & $\begin{array}{r}30 \\
2 \\
2 \\
3 \\
6\end{array}$ & & $\begin{array}{l}401.45 \\
413.20 \\
441.37 \\
490.65 \\
495.71\end{array}$ & $\begin{array}{c}z^{3} \mathrm{H}_{5}^{\circ}-e^{3} \mathrm{H}_{5} \\
x^{3} \mathrm{~F}_{3}^{\circ}-g^{3} \mathrm{P}_{2} \\
y^{3} \mathrm{D}_{1}^{\circ}-g^{3} \mathrm{P}_{0} \\
2^{3} \mathrm{H}_{1}^{\circ}-f^{3} \mathrm{~F}_{4}\end{array}$ & & \\
\hline $\begin{array}{l}65.62 \\
64.75 \\
62.73 \\
61.66 \\
61.36\end{array}$ & $\begin{array}{l}4 \\
3 \\
1 \mathrm{~h} \\
3 \\
4\end{array}$ & & $\begin{array}{l}503.59 \\
515.83 \\
544.29 \\
559.38 \\
563.61\end{array}$ & $\begin{array}{c}y^{1} \mathrm{~F}_{3}^{\circ}-i^{1} \mathrm{D}_{2} \\
2^{8} \mathrm{G}_{3}^{\circ}-g^{3} \mathrm{~F}_{3} \\
2^{1} \mathrm{~F}_{3}^{\circ}-f^{3} \mathrm{G}_{4} \\
y^{1} \mathrm{D}_{2}^{\circ}-i^{1} \mathrm{D}_{2}\end{array}$ & & \\
\hline $\begin{array}{l}49.61 \\
47.36 \\
44.70 \\
42.27 \\
40.15\end{array}$ & $\begin{array}{l}1 \\
4 \\
1 \\
1 \\
1\end{array}$ & & $\begin{array}{l}730.18 \\
762.25 \\
800.23 \\
834.96 \\
865.37\end{array}$ & $\begin{array}{l}y^{3} \mathrm{D}_{1}^{\circ}-g^{3} \mathrm{P}_{1} \\
2^{3} \mathrm{~F}_{3}^{\circ}-f^{1} \mathrm{~F}_{3} \\
y^{3} \mathrm{~F}_{3}^{\circ}-f^{1} \mathrm{G}_{4} \\
2^{1} \mathrm{G}_{4}^{\circ}-f^{3} \mathrm{G}_{5} \\
x^{3} \mathrm{~F}_{2}^{\circ}-g^{3} \mathrm{P}_{2}\end{array}$ & & \\
\hline $\begin{array}{l}39.00 \\
36.66 \\
31.94 \\
31.52 \\
20.01\end{array}$ & $\begin{array}{l}5 \\
1 \\
8 \\
4 \\
7\end{array}$ & & $\begin{array}{r}881.87 \\
915.48 \\
983.47 \\
3,7989.54 \\
3,8156.42\end{array}$ & $\begin{array}{l}z^{3} \mathrm{P}_{2}^{\circ}-f^{3} \mathrm{P}_{1} \\
z^{1} \mathrm{~F}_{3}^{\circ}-g^{3} \mathrm{D}_{3} \\
y^{1} \mathrm{~F}_{3}^{\circ}-f^{3} \mathrm{P}_{2} \\
2^{3} \mathrm{~F}_{2}^{\circ}-f^{1} \mathrm{~F}_{3} \\
2^{3} \mathrm{H}_{4}^{0}-e^{3} \mathrm{H}_{5}\end{array}$ & & \\
\hline $\begin{array}{l}17.29 \\
16.32 \\
13.09 \\
10.34 \\
04.18\end{array}$ & $\begin{array}{c}1 \\
7 \\
4 \\
150 \\
1\end{array}$ & & $\begin{array}{l}196.07 \\
210.23 \\
257.46 \\
297.76 \\
388.35\end{array}$ & $\begin{array}{c}2^{3} \mathrm{G}_{i}^{\circ}-g^{1} \mathrm{G}_{4} \\
2^{3} \mathrm{H}_{4}^{\circ}--^{1} \mathrm{G}_{4} \\
2^{3} \mathrm{H}_{5}-e^{3} \mathrm{H}_{6} \\
a^{1} \mathrm{~S}_{0}-x^{1} \mathrm{P}_{1}^{\circ} \\
y^{3} \mathrm{D}_{1}^{\circ}-h^{3} \mathrm{D}_{1}\end{array}$ & & - \\
\hline $\begin{array}{r}02.87 \\
01.79 \\
00.86 \\
2,600.33 \\
2,596.32\end{array}$ & $\begin{array}{l}1 \\
5 \\
2 \\
4 \\
3\end{array}$ & & $\begin{array}{l}407.66 \\
423.60 \\
437.34 \\
445.18 \\
504.55\end{array}$ & $\begin{array}{l}z^{3} \mathrm{P}_{1}^{0}-f^{3} \mathrm{P}_{1} \\
y^{3} \mathrm{P}_{1}^{0}-\mathrm{f}^{1} \mathrm{~S}_{0} \\
2^{3} \mathrm{P}_{0}^{0}-f^{3} \mathrm{P}_{1} \\
2^{1} \mathrm{D}_{2}^{0}-g^{3} \mathrm{~F}_{2}\end{array}$ & & \\
\hline $\begin{array}{l}96.08 \\
92.87 \\
86.35 \\
82.96 \\
82.55\end{array}$ & $\begin{array}{l}20 \\
3 \mathrm{~h} \\
10 \\
8 \\
6\end{array}$ & & $\begin{array}{l}508.11 \\
555.78 \\
652.97 \\
703.70 \\
709.85\end{array}$ & $\begin{array}{c}2^{1} \mathrm{G}_{4}^{0}-e^{3} \mathrm{H}_{4} \\
y^{3} \mathrm{D}_{1}^{\circ}-h^{3} \mathrm{D}_{2} \\
2^{3} \mathrm{D}_{1}^{0}-f^{3} \mathrm{P}_{0} \\
y^{3} \mathrm{~F}_{4}^{0}-g^{3} \mathrm{~F}_{3} \\
2^{3} \mathrm{~F}_{2}^{0}-f^{3} \mathrm{G}_{3}\end{array}$ & & \\
\hline
\end{tabular}


TABLE 4.-The first spark spectrum of lanthanum (La II)-Continued

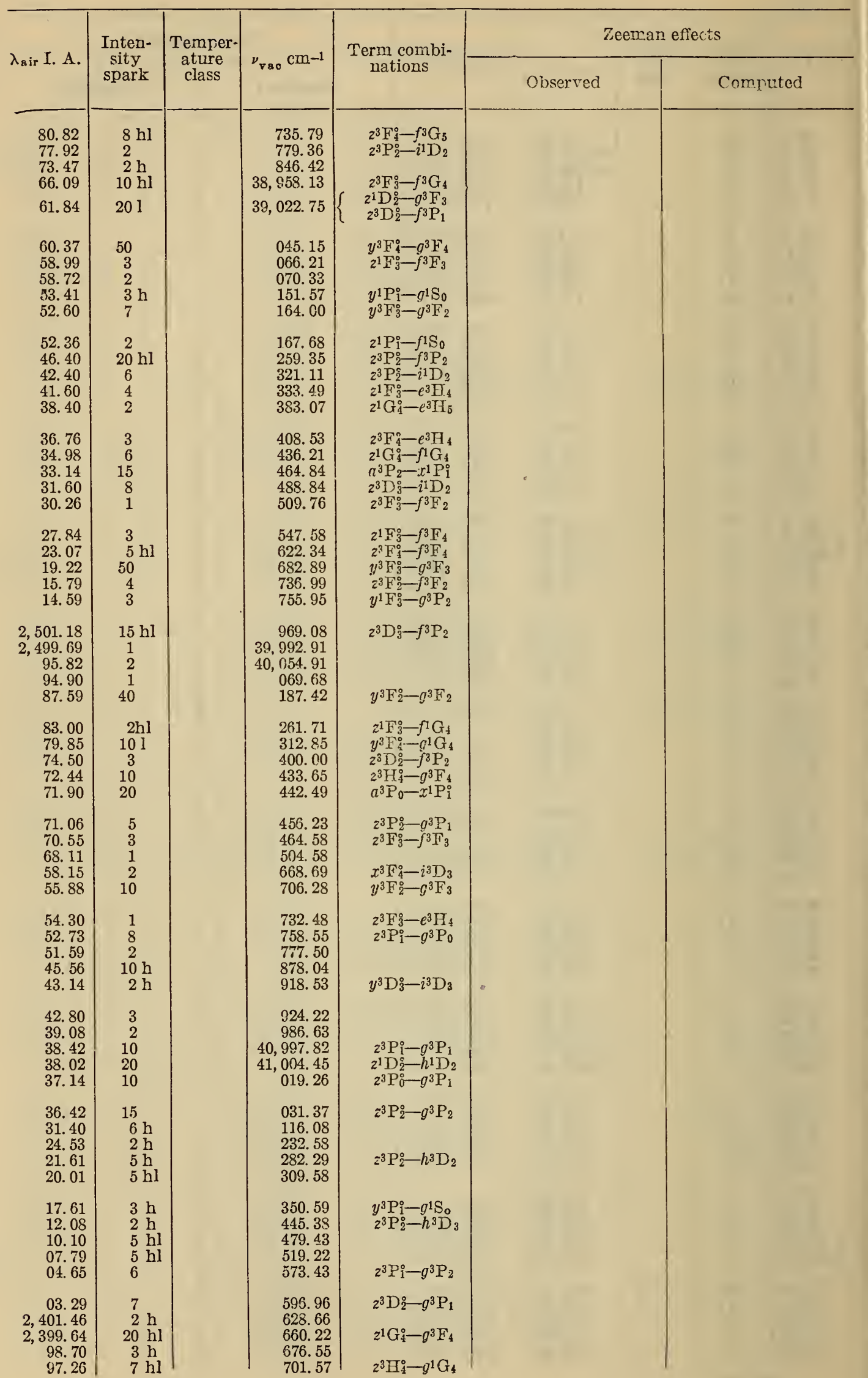


TABLE 4.-The first spark spectrum of lanthanum (La II)-Continued

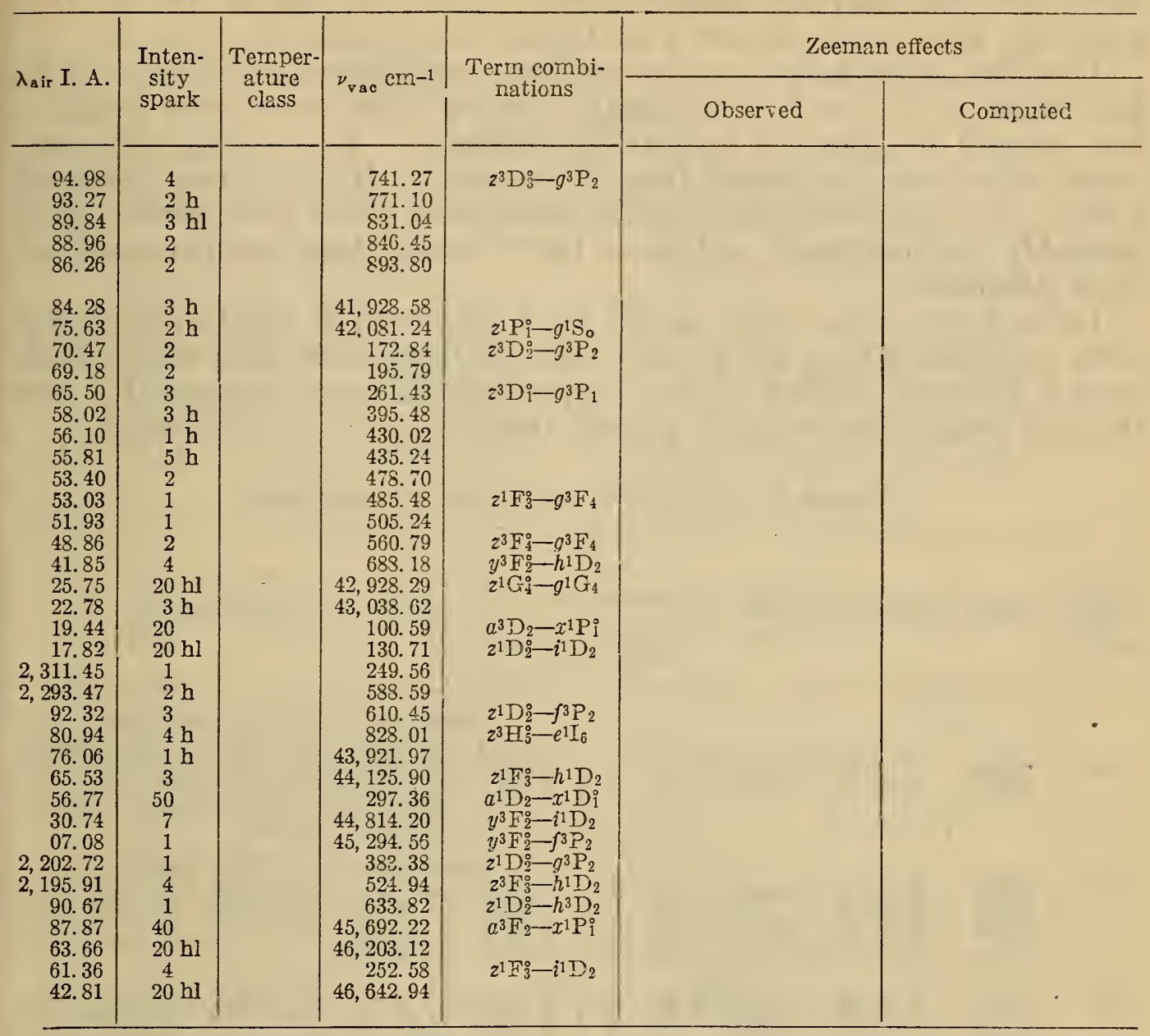

\section{THE SPECTRUM OF NEUTRAL LANTHANUM (La I)}

As in Sc and Y, the normal state of the neutral La atom is represented by a doublet-D term associated with the electron configuration $d s^{2}$. In each case, the next lowest energy is represented by a quartet-F term arising from $d^{2} s$, but in La it is only about one-third as high as in Sc or Y. The remaining metastable low terms also come from $d^{2} s$, and are exactly analogous to those in $\mathrm{Sc}$ and $\mathrm{Y}$. The ${ }^{2} \mathrm{G}$ is narrow and inverted in all three spectra, and the predicted ${ }^{2} \mathrm{~S}$ has been found in none. The odd levels are very numerous, and it is difficult to group them into terms. Indeed, parts of the tangle could not have been unraveled without the clues obtained from the new measurements of Zeeman effects.

The triads of quartet terms obtained by the addition of a $6 p$ electron to the ${ }^{3} \mathrm{D},{ }^{3} \mathrm{~F}$, and ${ }^{3} \mathrm{P}$ terms of La Ir can be securely identified. They all lie lower than the corresponding terms of Y r. Three additional odd quartet terms, $x^{4} \mathrm{~F}^{\circ}, y^{4} \mathrm{G}^{\circ}$, and $w^{4} \mathrm{D}^{\circ}$, at higher levels can come only from the addition of a $\Delta f$ electron to the same limits. The doublet terms arising from the addition of a $6 p$ electron to the various limits have also been certainly or probably identified by the intensities of their combinations and comparison of their levels with Y I. A number of supernumerary doublet terms must also arise from the $4 f$ electron, which in this case is less firmly bound than the $6 p$. 
Many of the numbered odd levels which have not been grouped into terms are probably of similar origin. The high terms, $10^{\circ}$ to $15^{\circ}$, probably arise from electrons of higher total quantum number.

The high even terms give much less conspicuous combinations in La I than in Sc I or Y I, and only a few of them have been foundbut enough to give the ionization potential. The unassigned even levels have been numbered from 30 onward. If the strong bands of $\mathrm{LaO}$ in the yellow and red could be eliminated more such terms could probably be identified, and some fairly strong high temperature arc lines classified.

Table 5 gives the terms of the La I spectrum in the same general form as Table 3 does for La II. The configuration from which each term is believed to arise is given opposite its lowest component. For the odd terms, the probable parent terms in La II are indicated.

TABLE 5.-Relative terms in the La I spectrum

\begin{tabular}{|c|c|c|c|c|c|c|c|}
\hline \multirow{2}{*}{$\begin{array}{l}\text { Elec- } \\
\text { tron } \\
\text { config- } \\
\text { uration }\end{array}$} & \multirow{2}{*}{ Term } & \multirow{2}{*}{ Level } & \multirow{2}{*}{$\begin{array}{l}\text { Level } \\
\text { separa- } \\
\text { tion }\end{array}$} & \multicolumn{3}{|c|}{$g$} & \multirow{2}{*}{ Combinations } \\
\hline & & & & $\begin{array}{l}\text { Adap } \\
\text { weig }\end{array}$ & & Landé & \\
\hline$d s^{2}$ & $\begin{array}{l}a^{2} \mathrm{D}_{11 / 3} \\
a^{2} \mathrm{D}_{21 / 5}\end{array}$ & $\begin{array}{r}0.00 \\
1,053.20\end{array}$ & $1,053.20$ & $\begin{array}{l}0.790 \\
1.203\end{array}$ & $\begin{array}{r}6 \\
14\end{array}$ & $\begin{array}{l}0.800 \\
1.200\end{array}$ & 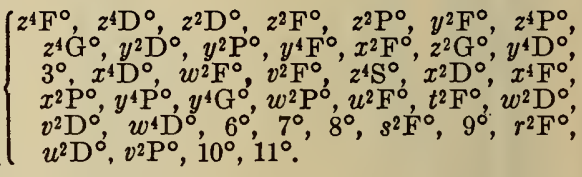 \\
\hline$d^{2} s$ & $\begin{array}{l}a^{4} \mathrm{~F}_{13 / 2} \\
a^{4} \mathrm{~F}_{2 / 3 / 2} \\
a^{4} \mathrm{~F}_{3 / 2} \\
a^{4} \mathrm{~F}_{4 / 2}\end{array}$ & $\begin{array}{l}2,668.20 \\
3,010.01 \\
3,494.58 \\
4,121.61\end{array}$ & $\begin{array}{l}341.81 \\
484.57 \\
627.03\end{array}$ & $\begin{array}{l}.411 \\
1.032 \\
1.222 \\
1.327\end{array}$ & $\begin{array}{r}20 \\
11 \\
10 \\
8\end{array}$ & $\begin{array}{l}.400 \\
1.029 \\
1.238 \\
1.333\end{array}$ & 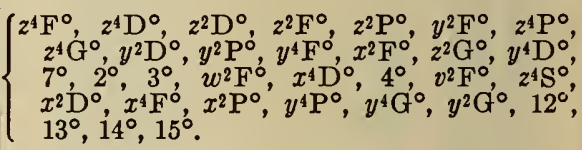 \\
\hline$d^{2} s$ & $\begin{array}{l}a^{4} \mathrm{P}_{1 / 2} \\
a^{4} \mathrm{P}_{1 / 2} \\
a^{4} \mathrm{P}_{23 / 2}\end{array}$ & $\begin{array}{l}7,231.36 \\
7,490.46 \\
7,679.94\end{array}$ & $\begin{array}{l}259.10 \\
189.48\end{array}$ & $\begin{array}{l}2.706 \\
1.69 \\
1.54\end{array}$ & $\begin{array}{r}10 \\
6 \\
4\end{array}$ & $\begin{array}{l}2.667 \\
1.733 \\
1.600\end{array}$ & 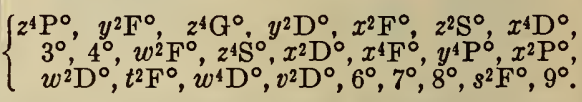 \\
\hline$d^{2} s$ & $\begin{array}{l}a^{2} \mathrm{~F}_{23 / 2} \\
a^{2} \mathrm{~F}_{3 / 2}\end{array}$ & $\begin{array}{l}7,011.90 \\
8,052.15\end{array}$ & $1,040.25$ & $\begin{array}{r}.90 \\
1.13\end{array}$ & $\begin{array}{l}31 / 2 \\
5\end{array}$ & $\begin{array}{r}.857 \\
1.143\end{array}$ & $\left\{\begin{array}{l}y^{2} \mathrm{~F}^{\circ}, z^{4} \mathrm{G}^{\circ}, z^{4} \mathrm{P}^{\circ}, y^{2} \mathrm{D}^{\circ}, y^{4} \mathrm{~F}^{\circ}, x^{2} \mathrm{~F}^{\circ}, z^{2} \mathrm{G}^{\circ}, \\
y^{4} \mathrm{D}^{\circ}, 1^{\circ}, 2^{\circ}, w^{2} \mathrm{~F}^{\circ}, v^{2} \mathrm{~F}^{\circ}, x^{2} \mathrm{D}^{\circ}, x^{4} \mathrm{~F}^{0}, x^{4} \mathrm{D}^{\circ}, \\
v^{2} \mathrm{~F}^{\circ}, y^{4} \mathrm{P}^{\circ}, y^{4} \mathrm{G}^{\circ}, y^{2} \mathrm{G}^{\circ}, w^{2} \mathrm{P}^{\circ}, u^{2} \mathrm{~F}^{\circ}, t^{2} \mathrm{~F}^{\circ}, \\
w^{2} \mathrm{D}^{\circ}, v^{2} \mathrm{D}^{\circ}, w^{4} \mathrm{D}^{\circ}, 7^{\circ}, s^{2} \mathrm{~F}^{\circ}, r^{2} \mathrm{~F}^{\circ}, u^{2} \mathrm{D}^{\circ}, \\
10^{\circ}, 11^{\circ} .\end{array}\right.$ \\
\hline$d^{2} s$ & $\begin{array}{l}b^{2} \mathrm{D}_{11 / 3} \\
b^{2} \mathrm{D}_{23 / 3}\end{array}$ & $\begin{array}{l}8,446.03 \\
9,183.77\end{array}$ & 737.74 & $\begin{array}{r}.89 \\
1.29\end{array}$ & $\begin{array}{l}3 \\
3\end{array}$ & $\begin{array}{r}.800 \\
1.200\end{array}$ & $\left\{\begin{array}{l}z^{4} \mathrm{G}^{\circ}, z^{4} \mathrm{P}^{\circ}, y^{2} \mathrm{D}^{\circ}, y^{2} \mathrm{P}^{\circ}, y^{4} \mathrm{~F}^{\circ}, z^{2} \mathrm{~S}^{\circ}, x^{4} \mathrm{D}^{\circ} \\
4^{\circ}, v^{2} \mathrm{~F}^{\circ}, z^{4} \mathrm{~S}^{\circ}, x^{2} \mathrm{D}^{\circ}, x^{4} \mathrm{~F}^{\circ} x^{2} \mathrm{O}^{\circ}, y^{4} \mathrm{P}^{\circ}, \\
w^{\circ} \mathrm{P}^{\circ}, u^{2} \mathrm{~F}^{\circ}, 5^{\circ}, w^{2} \mathrm{D}^{\circ}, s^{2} \mathrm{~F}^{\circ}, 9^{\circ}, r^{2} \mathrm{~F}^{\circ}, u^{2} \mathrm{D}^{\circ} .\end{array}\right.$ \\
\hline$d^{2} s$ & $\begin{array}{l}a^{2} \mathrm{P}_{1 / 1} \\
a^{2} \mathrm{P}_{1 / 2}\end{array}$ & $\begin{array}{l}9,044.21 \\
9,719.44\end{array}$ & 675.23 & $\begin{array}{r}.67 \\
1.32\end{array}$ & $\begin{array}{l}1 \\
2\end{array}$ & $\begin{array}{r}.667 \\
1.333\end{array}$ & $\left\{\begin{array}{c}y^{2} \mathrm{D}^{\circ}, y^{2} \mathrm{P}^{\circ}, y^{4} \mathrm{~F}^{\circ}, x^{2} \mathrm{~F}^{\circ}, z^{2} \mathrm{~S}^{\circ}, x^{4} \mathrm{D}^{\circ}, w^{2} \mathrm{~F}^{\circ}, 4^{\circ}, 4^{\circ} \mathrm{F}^{\circ}, x^{2} \mathrm{D}^{\circ}, x^{4} \mathrm{~F}^{\circ}, x^{2} \mathrm{P}^{\circ}, y^{4} \mathrm{P}^{\circ}, w^{2} \mathrm{P}^{\circ}, 5^{\circ}, \\
w^{2} \mathrm{D}^{\circ}, w^{2} \mathrm{P}^{\circ}, v^{2} \mathrm{D}^{\circ}, u^{2} \mathrm{D}^{\circ}, v^{2} \mathrm{P}^{\circ} .\end{array}\right.$ \\
\hline$d^{2} s$ & $\begin{array}{l}a^{2} \mathrm{G}_{413 / 5} \\
a^{2} \mathrm{G}_{83 / 2}\end{array}$ & $\begin{array}{l}9,919.94 \\
9,960.96\end{array}$ & -41.02 & $\begin{array}{r}1.12 \\
.91\end{array}$ & $\begin{array}{l}1 \\
1\end{array}$ & $\begin{array}{r}1.111 \\
.889\end{array}$ & $\left\{\begin{array}{c}y^{4} \mathrm{~F}^{\circ}, x^{2} \mathrm{~F}^{\circ}, z^{2} \mathrm{G}^{\circ}, y^{2} \mathrm{G}^{\circ}, w^{2} \mathrm{~F}^{\circ}, z^{2} \mathrm{H}^{\circ}, x^{4} \mathrm{~F}^{\circ}, \\
y^{2} \mathrm{G}^{\circ}, u^{2} \mathrm{~F}^{\circ}, y^{4} \mathrm{G} \mathrm{o}^{\circ}, w^{2} \mathrm{D}^{\circ}, t^{2} \mathrm{~F}^{\circ}, s^{2} \mathrm{~F}^{\circ}, 9^{\circ}, \\
r^{2} \mathrm{~F}^{\circ}, y^{2} \mathrm{H}^{\circ} .\end{array}\right.$ \\
\hline$a^{3} \mathrm{D} . p$ & 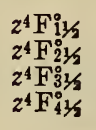 & $\begin{array}{l}13,260.36 \\
13,631.08 \\
15,019.55 \\
16,243.25\end{array}$ & $\begin{array}{r}370.72 \\
1,388.47 \\
1,223.70\end{array}$ & $\begin{array}{l}1.08 \\
1.21\end{array}$ & & $\begin{array}{l}\text { 1. } 029 \\
\text { 1. } 238\end{array}$ & $a^{2} \mathrm{D}, a^{4} \mathrm{~F}, 31,32,35, e^{4} \mathrm{~F}, e^{4} \mathrm{D}$ \\
\hline$a^{3} \mathrm{D} \cdot p$ & 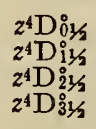 & $\begin{array}{l}14,095.70 \\
14,708.96 \\
15,503.67 \\
16,099.28\end{array}$ & $\begin{array}{l}613.26 \\
794.71 \\
595.61\end{array}$ & & & & $a^{2} \mathrm{D}, a^{4} \mathrm{~F}, 31,32,33,34, e^{4} \mathrm{~F}, e^{4} \mathrm{D}$ \\
\hline$a^{1} \mathrm{D} . p$ & $\begin{array}{l}z^{2} \mathrm{D}^{2} 13 / 5 \\
z^{2} \mathrm{D}_{1 / 4 / 5}^{2}\end{array}$ & $\begin{array}{l}14,804.10 \\
15,031.65\end{array}$ & -227.55 & $\begin{array}{r}1.08 \\
.97\end{array}$ & & $\begin{array}{r}1.200 \\
.800\end{array}$ & \}$a^{2} \mathrm{D}, a^{4} \mathrm{~F}, 31, e^{2} \mathrm{~F}$ \\
\hline$a^{1} \mathrm{D} . p$ & $\begin{array}{l}z^{2} \mathrm{~F}_{21 / 5}^{0} \\
z^{2} \mathrm{~F}_{3 / 2}^{2}\end{array}$ & $\begin{array}{l}15,196.80 \\
16,538.44\end{array}$ & $1,341.64$ & $\begin{array}{r}.90 \\
1.19\end{array}$ & & $\begin{array}{r}.857 \\
1.143\end{array}$ & \}$a^{2} \mathrm{D}, a^{4} \mathrm{~F}, e^{4} \mathrm{D}, e^{2} \mathrm{~F}$ \\
\hline$a^{1} \mathrm{~S} . p$ & $\begin{array}{l}z^{2} \mathrm{P}^{\circ}{ }^{2} \mathrm{P}_{1 / 2}^{1 / 3} \\
\mathrm{P}_{1 / 3}\end{array}$ & $\begin{array}{l}15,219.90 \\
16,280.20\end{array}$ & $1,060.30$ & & & & \\
\hline
\end{tabular}


TABLE 5.-Relative terms in the La I spectrum-Continued

\begin{tabular}{|c|c|c|c|c|c|c|}
\hline \multirow{2}{*}{$\begin{array}{l}\text { Elec- } \\
\text { tron } \\
\text { config- } \\
\text { uration }\end{array}$} & \multirow{2}{*}{ Term } & \multirow{2}{*}{ Level } & \multirow{2}{*}{$\begin{array}{l}\text { Level } \\
\text { separa- } \\
\text { tion }\end{array}$} & \multicolumn{2}{|c|}{$g$} & \multirow{2}{*}{ Combinations } \\
\hline & & & & $\begin{array}{c}\text { Adapted } \\
\text { weight }\end{array}$ & Landé & \\
\hline$a^{3} \mathrm{D} . p$ & $\begin{array}{l}y^{2} \mathrm{~F}_{21 / 3}^{\circ} \\
y^{2} \mathrm{~F}_{31 / 2}^{\circ}\end{array}$ & $\begin{array}{l}16,856.82 \\
17,910.18\end{array}$ & $1,053.36$ & $\begin{array}{l}0.83 \\
1.08\end{array}$ & $\begin{array}{l}0.857 \\
1.143\end{array}$ & $a^{2} \mathrm{D}, a^{4} \mathrm{~F}, a^{2} \mathrm{~F}, a^{4} \mathrm{P}$ \\
\hline$a^{3} \mathrm{D} . p$ & $\begin{array}{l}z^{4} \mathrm{P}^{\circ}{ }^{1 / 2} \\
z^{4} \mathrm{P}_{13 / 2}^{0} \\
z^{4} \mathrm{P}_{23 / 2}^{\circ}\end{array}$ & $\begin{array}{l}17,567.56 \\
17,797.30 \\
18,157.00\end{array}$ & $\begin{array}{l}229.74 \\
359.70\end{array}$ & 1.20 & 1. 600 & $a^{2} \mathrm{D}, a^{4} \mathrm{~F}, a^{2} \mathrm{~F}, a^{4} \mathrm{P}, b^{2} \mathrm{D}$ \\
\hline$a^{3} \mathbf{F} \cdot p$ & $\begin{array}{l}z^{4} \mathrm{G}_{21 / 2}^{\circ} \\
2^{4} \mathrm{G}_{31 / 2}^{0} \\
2^{4} \mathrm{G}_{1 / 2}^{0} \\
z^{4} \mathrm{G}_{5 / 2}^{0}\end{array}$ & $\begin{array}{l}17,947.16 \\
18,603.95 \\
19,129.34 \\
20,117.40\end{array}$ & $\begin{array}{l}656.79 \\
525.39 \\
988.06\end{array}$ & $\left\{\begin{array}{l}1.07 \\
1.06 \\
1.15 \\
1.27\end{array}\right.$ & $\begin{array}{r}.571 \\
.984 \\
1.172 \\
1.273\end{array}$ & $a^{2} \mathrm{D}, a^{4} \mathrm{~F}, a^{2} \mathrm{~F}, a^{4} \mathrm{P}, b^{2} \mathrm{D}, e^{4} \mathrm{~F}, 35$ \\
\hline$a^{3} \mathrm{D} . p$ & $\begin{array}{l}y^{2} \mathrm{D}_{11 / 2}^{\mathrm{i}} \\
y^{2} \mathrm{D}_{21 / 2}^{1}\end{array}$ & $\begin{array}{l}18,172.39 \\
19,379.44\end{array}$ & \} $1,207.05$ & $\left\{\begin{array}{r}.83 \\
1.19\end{array}\right.$ & $\begin{array}{r}.800 \\
1.200\end{array}$ & $a^{2} \mathrm{D}, a^{4} \mathrm{~F}, a^{2} \mathrm{~F}, a^{4} \mathrm{P}, b^{2} \mathrm{D}, a^{2} \mathrm{P}, a^{4} \mathrm{~F}$ \\
\hline$a^{1} \mathrm{D} \cdot p$ & $\begin{array}{l}y^{2} \mathrm{P}_{i 1 / 2}^{0} \\
y^{2} \mathrm{P}_{1 / 2}^{0}\end{array}$ & $\begin{array}{l}20,019.00 \\
20,197.38\end{array}$ & \}-178.38 & $\left\{\begin{array}{r}1.05 \\
.60\end{array}\right.$ & $\begin{array}{r}1.333 \\
.667\end{array}$ & $a^{2} \mathrm{D}, a^{4} \mathrm{~F}, b^{2} \mathrm{D}, a^{2} \mathrm{P}, e^{4} \mathrm{~F}$ \\
\hline$a^{3} \mathrm{~F} \cdot p$ & $\begin{array}{l}y^{4} \mathrm{~F}_{11 / 2}^{0} \\
y^{4} \mathrm{~F}_{21 / 2}^{\circ} \\
y^{4} \mathrm{~F}_{31 / 2}^{21} \\
y^{4} \mathrm{~F}_{41 / 2}^{0}\end{array}$ & $\begin{array}{l}20,083.02 \\
20,338.30 \\
20,763.31 \\
21,384.06\end{array}$ & $\begin{array}{l}255.28 \\
425.01 \\
620.75\end{array}$ & $\left\{\begin{array}{l}.78 \\
1.01 \\
1.18 \\
1.28\end{array}\right.$ & $\begin{array}{l}.400 \\
1.029 \\
1.238 \\
1.333\end{array}$ & $a^{2} \mathrm{D}, a^{4} \mathrm{~F}, a^{2} \mathrm{~F}, a^{2} \mathrm{P}, b^{2} \mathrm{D}, a^{2} \mathrm{G}, e^{4} \mathrm{~F}, e^{2} \mathrm{~F}$ \\
\hline$a^{3} \mathrm{~F} \cdot p$ & $\begin{array}{l}x^{2} \mathrm{~F}_{23 / 5}^{\circ} \\
x^{2} \mathrm{~F}_{31 / 5}^{\circ}\end{array}$ & $\begin{array}{l}20,972.22 \\
21,662.61\end{array}$ & 690.39 & $\left\{\begin{array}{r}.90 \\
1.13\end{array}\right.$ & $\begin{array}{r}.857 \\
1.143\end{array}$ & $a^{2} \mathrm{D}, a^{4} \mathrm{~F}, a^{2} \mathrm{~F}, a^{4} \mathrm{P}, a^{2} \mathrm{P}, a^{2} \mathrm{G}, e^{2} \mathrm{~F}$ \\
\hline$a^{3} F \cdot p$ & $\begin{array}{l}z^{2} \mathrm{G}_{31 / 2}^{0} \\
z^{2} \mathrm{G}_{41 / 2}^{\circ}\end{array}$ & $\begin{array}{l}21,447.92 \\
22,285.85\end{array}$ & 837.93 & $\left\{\begin{array}{l}1.08 \\
1.13\end{array}\right.$ & $\begin{array}{r}.889 \\
1.111\end{array}$ & $a^{2} \mathrm{D}, a^{4} \mathrm{~F}, a^{2} \mathrm{~F}, a^{2} \mathrm{G}, e^{2} \mathrm{~F}$ \\
\hline$a^{3} \mathrm{~F} \cdot p$ & 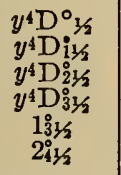 & $\begin{array}{l}22,246.64 \\
22,439.37 \\
22,804.26 \\
23,303.31 \\
23,221.16 \\
23,466.85\end{array}$ & $\begin{array}{l}192.73 \\
364.89 \\
499.05\end{array}$ & $\begin{array}{l}.03 \\
1.21 \\
1.35 \\
1.22 \\
1.11 \\
1.12\end{array}$ & $\begin{array}{l}.000 \\
1.200 \\
1.371 \\
1.429 \\
1.111\end{array}$ & $\begin{array}{l}a^{2} \mathrm{D}, a^{4} \mathrm{~F}, a^{2} \mathrm{~F} \\
a^{4} \mathrm{~F}, a^{2} \mathrm{~F} \\
a^{4} \mathrm{~F}, a^{2} \mathrm{~F}, a^{2} \mathrm{G}\end{array}$ \\
\hline$a \circ \mathrm{P} . p$ & $\begin{array}{l}z^{2} \mathrm{~S}^{\circ} 1 / 2 \\
3_{213 / 2}^{\circ} \\
4_{11 / 2}^{1}\end{array}$ & $\begin{array}{l}23,260.90 \\
23,549.42 \\
24,173.86\end{array}$ & & $\begin{array}{r}1.87 \\
.72\end{array}$ & 2. 000 & $\begin{array}{l}a^{4} \mathrm{P}, b^{2} \mathrm{P}, a^{2} \mathrm{P} . \\
a^{4} \mathrm{~F}, a^{4} \mathrm{P} . \\
a^{4} \mathrm{~F}, a^{4} \mathrm{P}, b^{2} \mathrm{D}, a^{2} \mathrm{P} .\end{array}$ \\
\hline$a^{3} \mathrm{P} . p$ & $\begin{array}{l}x^{4} \mathrm{D}^{\circ} 1 / 2 \\
x^{4} \mathrm{D}_{1}^{0} 1 / 2 \\
x^{4} \mathrm{D}_{21 / 2}^{0} \\
x^{4} \mathrm{D}_{31 / 2}^{0}\end{array}$ & $\begin{array}{l}23,528.38 \\
23,704.76 \\
24,046.06 \\
25,083.42\end{array}$ & $\begin{array}{r}176.38 \\
341.30 \\
1,037.36\end{array}$ & $\left\{\begin{array}{l}.00 \\
1.15 \\
1.22 \\
1.41\end{array}\right.$ & $\begin{array}{l}.000 \\
1.200 \\
1.371 \\
1.429\end{array}$ & $a^{2} \mathrm{D}, a^{4} \mathrm{~F}, a^{2} \mathrm{~F}, a^{4} \mathrm{P}, b^{2} \mathrm{D}, a^{2} \mathrm{P}$ \\
\hline$a^{3} \mathrm{D} . f$ & $\begin{array}{l}w^{2} \mathrm{~F}_{21 / 2}^{\circ} \\
w^{2} \mathrm{~F}_{31 / 2}^{\circ}\end{array}$ & $\begin{array}{l}23,875.00 \\
24,409.70\end{array}$ & 534. 70 & $\left\{\begin{array}{r}.94 \\
1.17\end{array}\right.$ & $\begin{array}{r}.857 \\
1.143\end{array}$ & $a^{2} \mathrm{D}, a^{4} \mathrm{~F}, a^{2} \mathrm{~F}, a^{4} \mathrm{P}, a^{2} \mathrm{P}, a^{2} \mathrm{G}$ \\
\hline & $\begin{array}{l}v^{2} \mathrm{~F}_{21 / 2}^{*}(?) \\
v^{2} \mathrm{~F}_{31 / 2}^{\circ}(?)\end{array}$ & $\begin{array}{l}24,507.89 \\
25,378.46\end{array}$ & 870.57 & $\left\{\begin{array}{l}1.20 \\
1.13\end{array}\right.$ & $\begin{array}{r}.857 \\
1.143\end{array}$ & $a^{2} \mathrm{D}, a^{4} \mathrm{~F}, a^{2} \mathrm{~F}, a^{4} \mathrm{P}, b^{2} \mathrm{D}, a^{2} \mathrm{P}$ \\
\hline $\begin{array}{l}a^{3} \mathrm{P} \cdot p \\
a^{3} \mathrm{~F} \cdot p\end{array}$ & $\begin{array}{l}z^{4} \mathrm{~S}_{1 / 1 / 2}^{i} \\
x^{2} \mathrm{D}_{11 / 2}^{\mathrm{i}} \\
x^{2} \mathrm{D}_{21 / 2}^{+1}\end{array}$ & $\begin{array}{l}24,639.27 \\
24,762.62 \\
25,218.25\end{array}$ & 455.63 & $\left\{\begin{array}{l}1.81 \\
.88 \\
1.25\end{array}\right.$ & $\begin{array}{r}2.000 \\
.800 \\
1.200\end{array}$ & $\begin{array}{l}a^{2} \mathrm{D}, a^{4} \mathrm{~F}, a^{4} \mathrm{P}, b^{2} \mathrm{D} \\
a^{2} \mathrm{D}, a^{4} \mathrm{~F}, a^{2} \mathrm{~F}, a^{4} \mathrm{P}, b^{2} \mathrm{D}\end{array}$ \\
\hline$a^{3} \mathrm{~F} . f$ & $\begin{array}{l}x^{4} \mathrm{~F}_{11 / 2}^{\circ} \\
x^{4} \mathrm{~F}_{21 / 2}^{\circ} \\
x^{4} \mathrm{~F}_{31 / 2}^{\circ} \\
x^{4} \mathrm{~F}_{41 / 2}^{\circ}\end{array}$ & $\begin{array}{l}24,910.39 \\
24,984.33 \\
25,380.33 \\
25,997.27\end{array}$ & $\begin{array}{r}73.94 \\
396.00 \\
616.94\end{array}$ & $\left\{\begin{array}{l}.73 \\
1.11 \\
1.23 \\
1.36\end{array}\right.$ & $\begin{array}{l}.400 \\
1.029 \\
1.238 \\
1.333\end{array}$ & $a^{2} \mathrm{D}, a^{4} \mathrm{~F}, a^{2} \mathrm{~F}, a^{2} \mathrm{G}$ \\
\hline$a^{1} \mathrm{G}, p$ & $\begin{array}{l}2^{2} \mathrm{H}_{41 / 2}^{\circ} \\
2^{2} \mathrm{H}_{53 / 5}^{\circ}\end{array}$ & $\begin{array}{l}25,089.50 \\
25,874.68\end{array}$ & 785. 18 & .94 & .909 & $a^{4} \mathrm{~F}, a^{2} \mathrm{G}$ \\
\hline$a^{3} \mathrm{D} \cdot p$ & $\begin{array}{l}x^{2} \mathrm{P}^{\circ}{ }^{1 / 2} \\
x^{2} \mathrm{P}_{1 / 2}^{1} \\
31 \\
32_{51 / 2}\end{array}$ & $\begin{array}{l}25,453.92 \\
25,950.39 \\
25,568.49 \\
25,881.53\end{array}$ & 496.47 & $\left\{\begin{array}{l}.92 \\
1.41\end{array}\right.$ & $\begin{array}{r}.667 \\
1.333\end{array}$ & $\begin{array}{l}a^{2} \mathrm{D}, a^{4} \mathrm{~F}, a^{4} \mathrm{P}, b^{2} \mathrm{D}, a^{2} \mathrm{P} \\
z^{2} \mathrm{D}^{\circ}, z^{4} \mathrm{~F}^{\circ}, z^{4} \mathrm{D}^{\circ} \\
z^{2} \mathrm{D}^{\circ}, z^{4} \mathrm{D}^{\circ}, z^{4} \mathrm{~F}^{\circ}\end{array}$ \\
\hline$a^{3} \mathrm{P} \cdot p$ & $\begin{array}{l}y^{4} \mathrm{P}^{\circ}{ }_{1 / 2} \\
y^{4} \mathrm{P}_{11 / 2}^{1} \\
y^{4} \mathrm{P}_{21 / 2}^{0}\end{array}$ & $\begin{array}{l}25,616.90 \\
25,643.02 \\
26,338.90\end{array}$ & $\begin{array}{r}26.12 \\
695.88\end{array}$ & $\left\{\begin{array}{l}2.29 \\
1.61 \\
1.54\end{array}\right.$ & $\begin{array}{l}2.667 \\
1.733 \\
1.600\end{array}$ & $a^{2} \mathrm{D}, a^{4} \mathrm{~F}, a^{2} \mathrm{~F}, a^{4} \mathrm{P}, b^{2} \mathrm{D}, a^{2} \mathrm{P}$ \\
\hline$a^{3} \mathrm{~F} . f$ & $\begin{array}{l}y^{4} \mathrm{G}_{21 / 2}^{\circ} \\
y^{4} \mathrm{G}_{31 / 2}^{0} \\
y^{4} \mathrm{G}_{41 / 2}^{\circ} 1 / 2 \\
y^{4} \mathrm{G}_{51 / 2}^{1}\end{array}$ & $\begin{array}{l}27,022.60 \\
27,455.34 \\
28,089.18 \\
28,743.21\end{array}$ & $\begin{array}{l}432.74 \\
633.84 \\
654.03\end{array}$ & $\left\{\begin{array}{r}.57 \\
.99 \\
1.16 \\
1.29\end{array}\right.$ & $\begin{array}{r}.571 \\
.984 \\
1.172 \\
1.273\end{array}$ & $a^{2} \mathrm{D}, a^{4} \mathrm{~F}, a^{2} \mathrm{~F}, a^{2} \mathrm{G}$ \\
\hline
\end{tabular}


TABLE 5.-Relative terms in the La I spectrum-Continued

\begin{tabular}{|c|c|c|c|c|c|c|}
\hline \multirow{2}{*}{$\begin{array}{l}\text { Elec- } \\
\text { uron } \\
\text { config- } \\
\text { uration }\end{array}$} & \multirow{2}{*}{ Term } & \multirow{2}{*}{ Level } & \multirow{2}{*}{$\begin{array}{l}\text { Level } \\
\text { separa- } \\
\text { tion }\end{array}$} & \multicolumn{2}{|l|}{$g$} & \multirow{2}{*}{ Combinations } \\
\hline & & & & $\begin{array}{l}\text { Adapted } \\
\text { weight }\end{array}$ & Landé & \\
\hline$a^{1} \mathrm{G} \cdot p$ & $\begin{array}{l}y^{2} \mathrm{G}_{31 / 2}^{g} \\
y^{2} \mathrm{G}_{41 / 2}^{+}\end{array}$ & $\begin{array}{l}27,132.50 \\
27,619.69\end{array}$ & 487.19 & $\begin{array}{l}0.95 \\
1.12\end{array}$ & $\begin{array}{l}0.889 \\
1.111\end{array}$ & \}$a^{4} \mathrm{~F}, a^{2} \mathrm{~F}, a^{2} \mathrm{G}$ \\
\hline $\begin{array}{l}b^{1} \mathrm{D} \cdot p \\
b^{1} \mathrm{D} \cdot p\end{array}$ & $\begin{array}{l}w^{2} \mathrm{P}_{11 / 2}^{\circ} \\
u^{2} \mathrm{~F}_{21 / 2}^{\circ} \\
u^{2} \mathrm{~F}_{31 / 2}^{\circ}\end{array}$ & $\begin{array}{l}27,225.27 \\
27,393.00 \\
28,039.54\end{array}$ & 646.54 & $\begin{array}{r}1.34 \\
.90 \\
1.13\end{array}$ & $\begin{array}{r}1.333 \\
.857 \\
1.143\end{array}$ & $\begin{array}{l}a^{2} \mathrm{D}, a^{2} \mathrm{~F}, b^{2} \mathrm{D}, a^{2} \mathrm{P} \\
a^{2} \mathrm{D}, a^{2} \mathrm{~F}, a^{2} \mathrm{G}\end{array}$ \\
\hline$f$ & $\begin{array}{r}t^{2} \mathrm{~F}_{21 / 2}^{o} \\
t^{2} \mathrm{~F}_{31 / 2}^{\circ} \\
5_{11 / 2}^{\circ}\end{array}$ & $\begin{array}{l}27,669.38 \\
28,543.10 \\
27,749.05\end{array}$ & 873. 72 & $\begin{array}{r}.88 \\
1.00\end{array}$ & $\begin{array}{r}.857 \\
1.143\end{array}$ & $\begin{array}{l}a^{2} \mathrm{D}, a^{2} \mathrm{~F}, a^{2} \mathrm{G} \\
b^{2} \mathrm{D}, a^{2} \mathrm{P} .\end{array}$ \\
\hline$b^{1} \mathrm{D} \cdot p$ & $\begin{array}{l}w^{2} \mathrm{D}_{1 / 2}^{\circ} \\
w^{2} \mathrm{D}_{21 / 2}^{8}\end{array}$ & $\begin{array}{l}27,968.53 \\
28,506.39\end{array}$ & 537.86 & .91 & .800 & \}$a^{2} \mathrm{D}, a^{2} \mathrm{~F}, a^{4} \mathrm{P}, b^{2} \mathrm{D}, a^{2} \mathrm{P}$ \\
\hline$a^{3} \mathrm{P} . f$ & $\begin{array}{l}w^{4} \mathrm{D}_{01 / 2}^{\circ} \\
w^{4} \mathrm{D}_{1 / 2}^{\circ} \\
w^{4} \mathrm{D}_{21 / 2}^{\circ} \\
w^{4} \mathrm{D}_{31 / 2}^{0}\end{array}$ & $\begin{array}{l}28,893.47 \\
29,199.53 \\
29,502.17 \\
29,894.91\end{array}$ & $\begin{array}{l}306.06 \\
302.64 \\
392.74\end{array}$ & $\begin{array}{l}0.00 \\
1.15 \\
1.29\end{array}$ & $\begin{array}{l}0.000 \\
1.200 \\
1.429\end{array}$ & $a^{2} \mathrm{D}, a^{2} \mathrm{~F}, a^{4} \mathrm{P}, b^{2} \mathrm{D}$ \\
\hline$f$ & $\begin{array}{r}v^{2} \mathrm{D}_{11 / 2}^{\circ} \\
v^{2} \mathrm{D}_{21 / 2}^{\circ} \\
33 \\
6^{\circ}{ }^{1 / 2} \\
7_{11 / 2}^{1}\end{array}$ & $\begin{array}{l}28,971.82 \\
29,775.57 \\
29,461.33 \\
29,564.92 \\
29,936.73\end{array}$ & 803.75 & $\begin{array}{r}.94 \\
1.13 \\
1.54\end{array}$ & $\begin{array}{r}.800 \\
1.200\end{array}$ & $\begin{array}{l}a^{2} \mathrm{D}, a^{2} \mathrm{~F}, a^{4} \mathrm{P}, b^{2} \mathrm{D}, a^{2} \mathrm{P} \\
z^{4} \mathrm{D} . \\
a^{2} \mathrm{D}, a^{4} \mathrm{P}, b^{2} \mathrm{D}, a^{2} \mathrm{P} \\
a^{2} \mathrm{D}, a^{2} \mathrm{~F}, a^{4} \mathrm{P} .\end{array}$ \\
\hline & $\begin{array}{l}81 / \sqrt{\circ} \\
34(?)\end{array}$ & $\begin{array}{l}30,417.47 \\
29,594.81\end{array}$ & & 1.54 & & $\begin{array}{l}a^{2} \mathrm{D}_{1} \quad a^{4} \mathrm{P}_{1} b^{2} \mathrm{D} \\
z^{4} \mathrm{D}^{\circ}\end{array}$ \\
\hline$a^{3} \mathrm{~F} . s$ & $\begin{array}{l}e^{4} \mathrm{~F}_{11 / 2} \\
e^{4} \mathrm{~F}_{21 / 2} \\
e^{4} \mathrm{~F}_{31 / 2} \\
e^{4} \mathrm{~F}_{41 / 2}\end{array}$ & $\begin{array}{l}29,874.89 \\
30,354.32 \\
31,059.69 \\
31,923.90\end{array}$ & $\begin{array}{l}479.43 \\
705.37 \\
864.21\end{array}$ & & & $z^{4} \mathrm{~F}^{\circ}, z^{4} \mathrm{D}^{\circ}, y^{4} \mathrm{~F}^{\circ}, z^{4} \mathrm{G}^{\circ}$. \\
\hline$a^{1} \mathrm{G} . p$ & $\begin{array}{l}s^{2} \mathrm{~F}_{21 / 2}^{\circ} \\
s^{2} \mathrm{~F}_{31 / 2}^{\circ} \\
9_{21 / 2}^{\circ}\end{array}$ & $\begin{array}{l}30,788.40 \\
30,964.82 \\
30,896.88\end{array}$ & 176. 42 & $\begin{array}{l}1.18 \\
1.45\end{array}$ & 1. 143 & $\left\{\begin{array}{l}a^{2} \mathrm{D}, a^{4} \mathrm{P}, a^{2} \mathrm{~F}, b^{2} \mathrm{D}, a^{2} \mathrm{G} \\
a^{2} \mathrm{D}, a^{4} \mathrm{P}, b^{2} \mathrm{D}, a^{2} \mathrm{G}\end{array}\right.$ \\
\hline$a^{3} \mathrm{~F} . s$ & $\begin{array}{l}e^{2} \mathrm{~F}_{21 / 2} \\
e^{2} \mathrm{~F}_{31 / 2}\end{array}$ & $\begin{array}{l}31,119.05 \\
32,108.58\end{array}$ & 989.50 & & & $z^{2} \mathrm{D}^{\circ}, z^{2} \mathrm{~F}^{\circ}, y^{4} \mathrm{~F}^{\circ}, z^{2} \mathrm{G}^{\circ}$. \\
\hline$a^{3} \mathrm{D} . s$ & $e^{4} D_{31 / 2}$ & $31,287.65$ & & & & $z^{4} \mathrm{~F}^{\circ}, z^{4} \mathrm{D}^{\circ}, z^{2} \mathrm{~F}^{\circ}$ \\
\hline$f$ & $\begin{array}{l}r^{2} \mathrm{~F}_{21 / 2}^{o} \\
r^{2} \mathrm{~F}_{31 / 2}^{o / 1}\end{array}$ & $\begin{array}{l}31,477.16 \\
32,140.60\end{array}$ & & $\begin{array}{r}.88 \\
1.18\end{array}$ & $\begin{array}{r}.857 \\
1.143\end{array}$ & $a^{2} \mathrm{D}, a^{2} \mathrm{~F}, b^{2} \mathrm{D}, a^{2} \mathrm{G}$. \\
\hline$a^{3} \mathrm{P} \cdot p$ & $\begin{array}{l}u^{2} \mathrm{D}_{11 / 2}^{i} \\
u^{2} \mathrm{D}_{21 / 2}^{\circ}\end{array}$ & $\begin{array}{l}31,751.68 \\
32,492.80\end{array}$ & 741. 12 & $\begin{array}{r}.82 \\
1.17\end{array}$ & $\begin{array}{r}.800 \\
1.200\end{array}$ & $a^{2} \mathrm{D}, a^{2} \mathrm{~F}, b^{2} \mathrm{D}, a^{2} \mathrm{P}$ \\
\hline$a^{3} \mathrm{P} . p$ & $\begin{array}{l}v^{2} \mathrm{P}^{\mathrm{o}} v^{1 / 2} \\
v^{2} \mathrm{P}_{11 / 2}^{1}\end{array}$ & $\begin{array}{l}32,290.25 \\
33,204.20\end{array}$ & 913.95 & 1.26 & 1. 333 & $a^{2} \mathrm{D}, b^{2} \mathrm{D}, a^{2} \mathrm{P}$ \\
\hline$a^{1} \mathrm{G} . f$ & $\begin{array}{c}y^{2} \mathrm{H}_{41 / 2}^{\circ} \\
y^{2} \mathrm{H}_{51 / 2}^{\circ} \\
35^{\circ} \\
10^{\circ} \\
11^{\circ} \\
12^{\circ}(?) \\
13^{\circ} \\
14^{\circ} \\
15^{\circ}\end{array}$ & $\begin{array}{l}32,410.76 \\
32,518.12 \\
33,286.50 \\
36,722.38 \\
37,731.90 \\
39,597.58 \\
39,631.27 \\
40,322.45 \\
40,343.40\end{array}$ & 107.36 & $\begin{array}{r}.93 \\
1.11\end{array}$ & $\begin{array}{r}.909 \\
1.091\end{array}$ & $\begin{array}{l}a^{2} \mathrm{G} . \\
z^{4} \mathrm{~F}^{\circ}, z^{2} \mathrm{~F}^{\circ}, y^{2} \mathrm{~F}^{\circ}, z^{4} \mathrm{G}^{\circ} . \\
a^{2} \mathrm{D}, a^{2} \mathrm{~F} . \\
a^{2} \mathrm{D}, a^{2} \mathrm{~F} . \\
a^{4} \mathrm{~F} . \\
a^{4} \mathrm{~F} . \\
a^{4} \mathrm{~F} . \\
a^{4} \mathrm{~F} .\end{array}$ \\
\hline
\end{tabular}

The spectral terms and combinations for La I are represented diagrammatically in Figure 2, which may be compared with corresponding diagrams for Sc I and Y I spectra in publications already referred to. ${ }^{25}$

Complete details of lines regarded as characteristic of neutral lanthanum atoms are given in Table 6 , the arrangement and notation being the same as that in Table 4 . Here again the Zeeman patterns computed from the adopted $g$ values are almost always in good agreement with observation. 


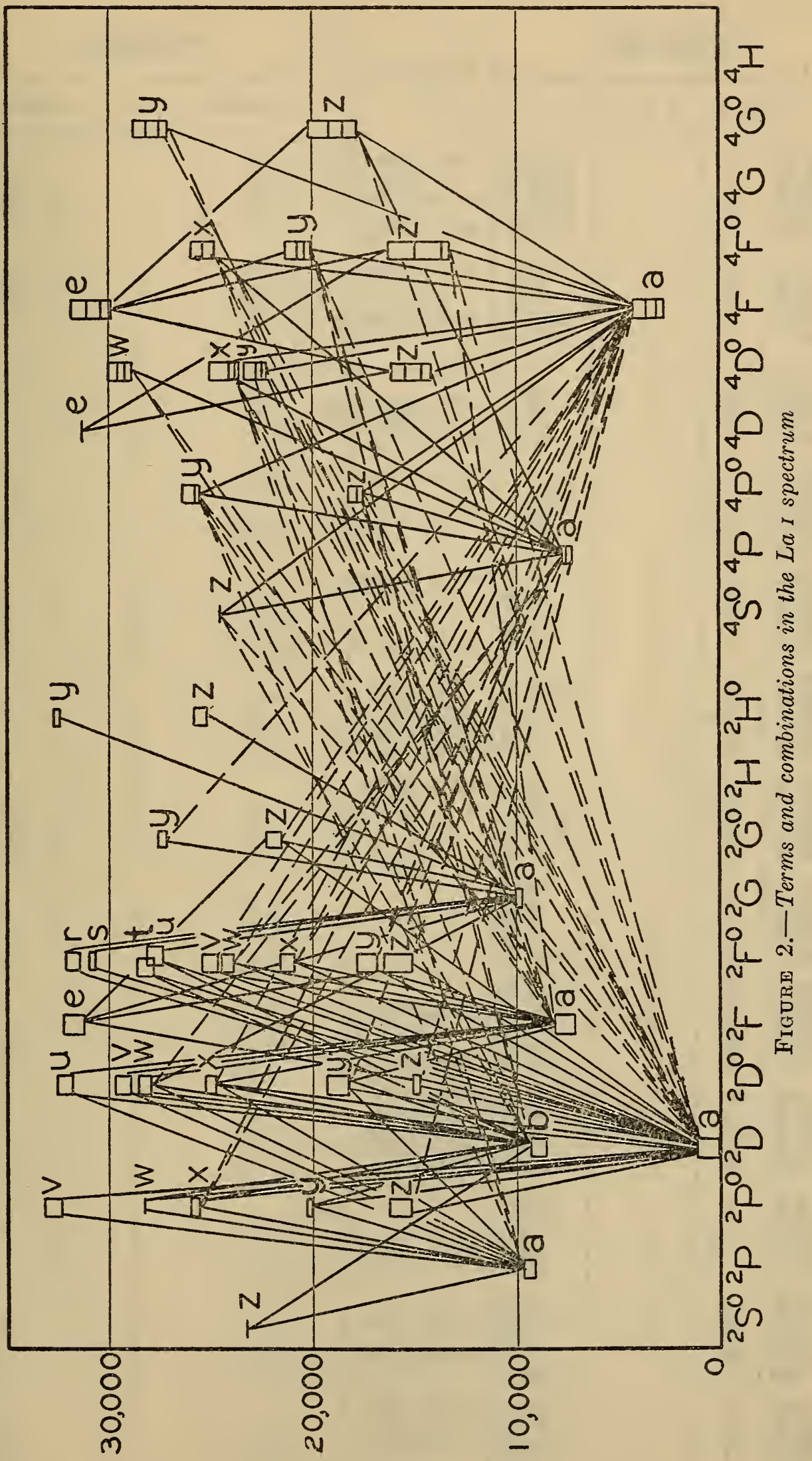


TABLE 6.-The arc spectrum of lanthanum ( $L a I)$

\begin{tabular}{|c|c|c|c|c|c|c|}
\hline \multirow{2}{*}{$\lambda_{\text {air I. A. }}$} & \multicolumn{2}{|c|}{$\begin{array}{l}\text { Intensities, tem- } \\
\text { perature class }\end{array}$} & \multirow{2}{*}{$\nu_{\mathrm{vac}} \mathrm{cm}^{-1}$} & \multirow{2}{*}{$\begin{array}{c}\text { Term combi- } \\
\text { nation }\end{array}$} & \multicolumn{2}{|c|}{ Zeeman effects } \\
\hline & B. S. & K. \& C. & & & Observed & Computed \\
\hline $\begin{array}{l}10,952.0 \\
10,739.66 \\
10,612.56 \\
10,552.41 \\
10,522.09\end{array}$ & $\begin{array}{r}1 \\
5 \\
10 \\
6 \\
10\end{array}$ & & $\begin{array}{l}9,128.25 \\
9,308.73 \\
9,420.21 \\
9,473.91 \\
9,501.21\end{array}$ & $\begin{array}{l}a^{2} \mathrm{P}_{1 / 2}-y^{2} \mathrm{D}_{1 / 2}^{0} \\
b^{2} \mathrm{D}_{21 / 2}-z^{4} \mathrm{G}_{31 / 2}^{0} \\
b^{2} \mathrm{D}_{13 / 2}-z^{4} \mathrm{G}_{21 / 2}^{0}\end{array}$ & & \\
\hline $\begin{array}{c}10,483.0 \\
61.69 \\
50.82 \\
23.4 \\
10,409.55\end{array}$ & $\begin{array}{r}2 \\
15 \\
20 \\
1 \\
3\end{array}$ & & $\begin{array}{c}36.6 \\
56.07 \\
66.01 \\
9,591.2 \\
9,603.93\end{array}$ & $\begin{array}{l}y^{4} \mathrm{~F}_{21 / 2}^{0}-e^{4} \mathrm{~F}_{13 / 3} \\
y^{4} \mathrm{~F}_{3 / 2}^{0}-e^{4} \mathrm{~F}_{23 / 5}\end{array}$ & & \\
\hline $\begin{array}{r}10,372.4 \\
57.70 \\
49.08 \\
37.20 \\
32.40\end{array}$ & $\begin{array}{r}1 \\
20 \\
40 \\
3 \\
2\end{array}$ & & $\begin{array}{l}38.4 \\
52.01 \\
60.05 \\
71.15 \\
75.64\end{array}$ & $\begin{array}{l}z^{4} \mathrm{~F}_{41 / 2}^{0}-32 \\
a^{2} \mathrm{P}_{11 / 3}-y^{2} \mathrm{D}_{21 / 2}^{0} \\
z^{2} \mathrm{G}_{31 / 2}^{0}-e^{2} \mathrm{~F}_{21 / 2} \\
y^{4} \mathrm{~F}_{41 / 2}^{0}-e^{4} \mathrm{~F}_{31 / 2}^{0}\end{array}$ & & \\
\hline $\begin{array}{r}30.3 \\
10,318.2 \\
10,294.68 \\
85.64 \\
81.34\end{array}$ & $\begin{array}{c}1 \\
2 \\
10 \mathrm{~d} \\
3 \\
10\end{array}$ & & $\begin{array}{r}77.6 \\
9,689.0 \\
9,711.09 \\
21.52 \\
23.69\end{array}$ & $\begin{array}{l}y^{2} \mathrm{P}_{11 / 2}^{0}-e^{4} \mathrm{~F}_{11 / 2} \\
b^{2} \mathrm{D}_{13 / 2}-2^{4} \mathrm{P}_{21 / 2}^{\circ}\end{array}$ & & \\
\hline $\begin{array}{l}78.52 \\
74.85 \\
34.78 \\
23.76 \\
19.83\end{array}$ & $\begin{array}{r}3 \\
10 \\
2 \\
1 \\
3\end{array}$ & & $\begin{array}{l}26.37 \\
29.84 \\
67.93 \\
78.46 \\
82.22\end{array}$ & $\begin{array}{l}b^{2} \mathrm{D}_{1 / 3}-y^{2} \mathrm{D}_{1 / 2}^{\circ} \\
2^{4} \mathrm{D}_{31 / 2}^{\circ}-32\end{array}$ & & \\
\hline $\begin{array}{r}10,209.85 \\
10,184.60 \\
77.74 \\
54.74 \\
43.38\end{array}$ & $\begin{array}{c}2 \\
20 \\
6 \mathrm{~b} \\
40 \\
2\end{array}$ & & $\begin{array}{r}9,791.78 \\
9,816.06 \\
22.67 \\
44.92 \\
55.95\end{array}$ & $\begin{array}{l}y^{4} \mathrm{~F}_{1 / 2}^{0}-e^{4} \mathrm{~F}_{11 / 2} \\
z^{2} \mathrm{G}_{41 / 3}^{0}-e^{2} \mathrm{~F}_{31 / 2} \\
a^{2} \mathrm{~F}_{21 / 2}-y^{2} \mathrm{~F}_{21 / 2}^{\circ} \\
y^{2} \mathrm{P}_{1 / 2}^{1}-e^{4} \mathrm{~F}_{1 / 1 / 2}\end{array}$ & & - \\
\hline $\begin{array}{r}41.20 \\
30.82 \\
10,111.9 \\
10,083.96 \\
66.77\end{array}$ & $\begin{array}{l}10 \\
5 \\
2 \mathrm{~h} \\
2 \\
6\end{array}$ & & $\begin{array}{r}58.06 \\
68.17 \\
9,886.6 \\
9,914.03 \\
30.95\end{array}$ & $a^{2} F_{33 / 2}-y^{2} F_{33 / 2}^{0}$ & & \\
\hline $\begin{array}{r}58.79 \\
54.82 \\
29.74 \\
14.45 \\
10,005.73\end{array}$ & $\begin{array}{r}2 \\
2 \\
2 \\
4 \\
50\end{array}$ & & $\begin{array}{r}34.84 \\
42.76 \\
67.62 \\
82.84 \\
9,991.54\end{array}$ & & & 71 \\
\hline $\begin{array}{r}9,988.47 \\
81.24 \\
80.38 \\
65.70 \\
32.72\end{array}$ & $\begin{array}{r}10 \\
6 \\
10 \\
3 \\
2\end{array}$ & & $\begin{array}{r}10,008.80 \\
016.05 \\
016.91 \\
031.67 \\
064.98\end{array}$ & $\begin{array}{l}y^{4} \mathrm{~F}_{23 / 5}^{0}-e^{4} \mathrm{~F}_{23 / 5} \\
2^{4} \mathrm{D}_{2 / 2}^{2}-31\end{array}$ & & 1 \\
\hline $\begin{array}{r}20.82 \\
9,911.08\end{array}$ & $\begin{array}{r}150 \\
3\end{array}$ & & $\begin{array}{l}077.06 \\
085.95\end{array}$ & $a^{4} P_{135}-2^{4} P_{3 / 2}$ & & \\
\hline $\begin{array}{r}9,881.24 \\
62.60 \\
52.58\end{array}$ & $\begin{array}{r}3 \\
100 \\
3 \\
6\end{array}$ & & $\begin{array}{l}117.42 \\
136.54 \\
146.85\end{array}$ & $\begin{array}{l}a^{4} \mathrm{P}_{21 / 3}-z^{4} \mathrm{P}_{13 / 5}^{0} \\
a^{4} \mathrm{~F}_{3 / 3}-2^{4} \mathrm{~F}_{21 / 2}^{0} \\
x^{2} \mathrm{~F}_{21 / 2}^{0}-e^{2} \mathrm{~F}_{21 / 2}\end{array}$ & & \\
\hline $\begin{array}{r}48.70 \\
42.0 \\
33.30 \\
05.2 \\
9,804.20\end{array}$ & $\begin{array}{l}4 \\
2 \\
3 \mathrm{~h} \\
11 \mathrm{~h} \\
2\end{array}$ & & $\begin{array}{l}150.84 \\
157.8 \\
166.74 \\
195.9 \\
196.92\end{array}$ & $b^{2} \mathrm{D}_{23 / 5}-y^{2} \mathrm{D}_{23 / 5}^{2}$ & & \\
\hline $\begin{array}{r}9,775.09 \\
72.24 \\
68.82 \\
37.09 \\
13.52\end{array}$ & $\begin{array}{c}8 \\
20 \\
3 \mathrm{~h} \\
100 \\
3\end{array}$ & & $\begin{array}{l}227.28 \\
230.26 \\
233.85 \\
267.20 \\
292.11\end{array}$ & $\begin{array}{l}a^{4} \mathrm{P}_{23 / 5}-y^{2} \mathrm{~F}_{3 / 2}^{\circ} \\
a^{4} \mathrm{P}_{23 / 2}-z^{4} \mathrm{G}_{21 / 5}^{\circ}\end{array}$ & & \\
\hline $\begin{array}{r}09.45 \\
9,706.48 \\
9,699.64 \\
96.7 \\
92.6\end{array}$ & $\begin{array}{r}10 \\
20 \\
20 \\
1 \\
2\end{array}$ & & $\begin{array}{l}296.42 \\
299.57 \\
306.84 \\
310.0 \\
314.3\end{array}$ & 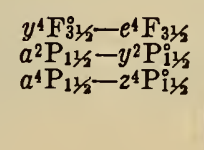 & & \\
\hline
\end{tabular}


TABLE 6.-The arc spectrum of lanthanum ( $L a I)$-Continued

\begin{tabular}{|c|c|c|c|c|c|c|}
\hline \multirow{2}{*}{$\lambda_{\text {air }}$ I. A. } & \multicolumn{2}{|c|}{$\begin{array}{l}\text { Intensities, tem- } \\
\text { perature class }\end{array}$} & \multirow{2}{*}{$\nu_{\nabla} \mathrm{BO}_{0} \mathrm{~cm}^{-1}$} & \multirow{2}{*}{$\begin{array}{l}\text { Term combi- } \\
\text { nation }\end{array}$} & \multicolumn{2}{|c|}{ Zeeman effects } \\
\hline & B. S. & K. \& C. & & & Observed & Computed \\
\hline $\begin{array}{r}72.04 \\
46.47 \\
40.81 \\
33.72 \\
9,631.84\end{array}$ & $\begin{array}{r}8 \\
3 \\
30 \\
40 \\
2\end{array}$ & & $\begin{array}{l}336.25 \\
363.65 \\
369.73 \\
377.36 \\
379.38\end{array}$ & $\begin{array}{l}a^{4} \mathrm{P}_{1 / 2}-z^{4} \mathrm{P}_{01 / 2}^{\circ} \\
a^{2} \mathrm{P}_{11 / 2}-y^{4} \mathrm{~F}_{1 / 2}^{011} \\
a^{2} \mathrm{G}_{31 / 2}-y^{4} \mathrm{~F}_{21 / 2}^{0}\end{array}$ & & \\
\hline $\begin{array}{r}9,570.38 \\
60.69 \\
42.06 \\
41.23 \\
9,528.0\end{array}$ & $\begin{array}{c}5 \\
10 \\
50 \\
20 \\
1 \mathrm{~h}\end{array}$ & & $\begin{array}{l}446.04 \\
456.63 \\
477.05 \\
477.96 \\
492.5\end{array}$ & $\begin{array}{l}x^{2} \mathrm{~F}_{31 / 2}^{0}-e^{2} \mathrm{~F}_{31 / 2} \\
a^{4} \mathrm{P}_{11 / 2}-2^{4} \mathrm{G}_{21 / 2}^{\circ} \\
a^{4} \mathrm{P}_{21 / 3}-2^{4} \mathrm{P}_{21 / 3}^{0} \\
a^{2} \mathrm{P}_{11 / 2}-y^{2} \mathrm{P}_{01 / 2}^{\circ} \\
a^{4} \mathrm{P}_{21 / 2}-y^{2} \mathrm{D}_{11 / 2}^{\circ}\end{array}$ & & \\
\hline $\begin{array}{c}9,485.15 \\
84.2 \\
76.98 \\
74.45 \\
67.25\end{array}$ & $\begin{array}{r}15 \\
1 \\
3 \\
5 \\
2\end{array}$ & & $\begin{array}{l}539.91 \\
541.0 \\
548.99 \\
551.81 \\
559.84\end{array}$ & $\begin{array}{l}y^{4} F_{41 / 2}^{0}-e^{4} F_{11 / 2} \\
z^{4} F_{31 / 2}^{\circ}-31 \\
a^{2} F_{31 / 2}^{\circ}-z^{4} G_{33 / 2}^{\circ}\end{array}$ & te & \\
\hline $\begin{array}{l}61.82 \\
57.62 \\
41.7 \\
38.30 \\
15.64\end{array}$ & $\begin{array}{r}60 \\
2 \\
1 \\
100 \\
3\end{array}$ & & $\begin{array}{l}565.89 \\
570.59 \\
588.4 \\
592.23 \\
617.71\end{array}$ & $\begin{array}{l}a^{4} \mathrm{P}_{1 / 2}-z^{4} \mathrm{P}_{1 / 2} \\
a^{4} \mathrm{~F}_{1 / 2}-z^{4} \mathrm{~F}_{1 / 2}\end{array}$ & $\mathrm{f}$ & \\
\hline $\begin{array}{c}9,412.65 \\
9,398.2 \\
90.56 \\
77.71 \\
76.10\end{array}$ & $\begin{array}{r}100 \\
1 \\
4 \\
3 \\
3\end{array}$ & & $\begin{array}{l}621.08 \\
637.4 \\
646.07 \\
660.66 \\
662.49\end{array}$ & $\begin{array}{l}a^{4} \mathrm{~F}_{21 / 2}-z^{4} \mathrm{~F}_{21 / 2}^{\circ} \\
z^{2} \mathrm{G}_{31 / 2}^{\circ}-e^{2} \mathrm{~F}_{31 / 2}\end{array}$ & . & \\
\hline $\begin{array}{r}72.57 \\
28.87 \\
24.5 \\
9.321 .9 \\
9,293.3\end{array}$ & $\begin{array}{l}30 \\
2 \\
1 \\
1 \mathrm{~h} \\
2 \mathrm{~h}\end{array}$ & & $\begin{array}{l}666.51 \\
716.47 \\
721.5 \\
724.5 \\
757.5\end{array}$ & $\begin{array}{l}a^{4} \mathrm{P}_{11 / 2}-z^{4} \mathrm{P}_{21 / 2}^{\circ} \\
y^{4} \mathrm{~F}_{21 / 2}^{0}-e^{4} \mathrm{~F}_{31 / 2} \\
y^{4} \mathrm{~F}_{41 / 2}^{0}-e^{2} \mathrm{~F}_{31 / 2}\end{array}$ & & \\
\hline $\begin{array}{r}87.5 \\
54.70 \\
50.06 \\
26.63 \\
9,219.64\end{array}$ & $\begin{array}{l}1 \mathrm{~h} \\
10 \\
20 \\
30 \\
10\end{array}$ & & $\begin{array}{l}764.2 \\
802.36 \\
807.78 \\
835.22 \\
843.44\end{array}$ & $\begin{array}{l}z^{2} \mathrm{D}_{21 / 2}^{\circ}-31 \\
a^{2} \mathrm{G}_{31 / 2}-y^{4} \mathrm{~F}_{31 / 2}^{\circ} \\
b^{2} \mathrm{D}_{21 / 2}-y^{2} \mathrm{P}_{11 / 2}^{\circ} \\
a^{2} \mathrm{G}_{41 / 2}-y^{4} \mathrm{~F}_{31 / 2}^{\circ}\end{array}$ & & \\
\hline $\begin{array}{r}9,172.88 \\
72.39 \\
57.13 \\
51.62 \\
43.78\end{array}$ & $\begin{array}{r}5 \\
10 \\
10 \\
6 \\
5\end{array}$ & & $\begin{array}{l}898.71 \\
899.30 \\
917.46 \\
924.03 \\
933.40\end{array}$ & $\begin{array}{l}b^{2} \mathrm{D}_{21 / 2}-y^{4} \mathrm{~F}_{11 / 2}^{\circ} \\
a^{4} \mathrm{P}_{21 / 2}-z^{4} \mathrm{G}_{31 / 2}^{\circ} \\
b^{2} \mathrm{D}_{11 / 2}-y^{2} \mathrm{D}_{21 / 2}^{0}\end{array}$ & & s \\
\hline $\begin{array}{r}42.24 \\
19.18 \\
9,109.25 \\
9,079.10 \\
58.63\end{array}$ & $\begin{array}{r}6 \\
20 \\
2 \\
50 \\
2\end{array}$ & & $\begin{array}{r}935.24 \\
962.89 \\
10,974.84 \\
11,011.29 \\
036.17\end{array}$ & $\begin{array}{l}a^{2} \mathrm{D}_{11 / 2}-2^{4} \mathrm{G}_{21 / 2}^{0} \\
a^{4} \mathrm{~F}_{11 / 2}-z^{4} \mathrm{~F}_{21 / 2}^{2} \\
a^{2} \mathrm{P}_{1 / 2}-y^{2} \mathrm{P}_{11 / 2}^{1} \\
a^{2} \mathrm{G}_{31 / 2}-x^{2} \mathrm{~F}_{21 / 2}^{2} \\
y^{4} \mathrm{~F}_{11 / 3}^{1}-e^{2} \mathrm{~F}_{21 / 3}\end{array}$ & & \\
\hline $\begin{array}{r}56.53 \\
46.97 \\
25.05 \\
9,008.26 \\
8,977.39\end{array}$ & $\begin{array}{l}5 \\
2 \\
4 \\
6 \\
2\end{array}$ & & $\begin{array}{l}038.73 \\
050.39 \\
077.23 \\
097.88 \\
136.04\end{array}$ & $\begin{array}{l}a^{2} \mathrm{P}_{1 / 3}-y^{4} \mathrm{~F}_{11 / 2}^{i} \\
a^{2} \mathrm{~F}_{31 / 2}-2^{4} \mathrm{G}_{41 / 2}^{\circ} \\
x^{2} \mathrm{~F}_{21 / 2}^{\circ}-e^{2} \mathrm{~F}_{31 / 2}\end{array}$ & & \\
\hline $\begin{array}{l}70.07 \\
65.41 \\
63.63 \\
57.74 \\
48.89\end{array}$ & $\begin{array}{r}3 \\
2 \\
10 \\
50 \\
2\end{array}$ & & $\begin{array}{l}145.13 \\
150.92 \\
153.13 \\
160.47 \\
171.50\end{array}$ & $\begin{array}{r}a^{2} \mathrm{~F}_{21 / 2}-z^{4} \mathrm{P}_{21 / 2}^{1} \\
a^{2} \mathrm{P}_{1 / 2}-y^{2} \mathrm{P}_{01 / 2}^{0} \\
a^{2} \mathrm{~F}_{21 / 2}-y^{2} \mathrm{D}_{11 / 2}^{0}\end{array}$ & & \\
\hline $\begin{array}{r}47.95 \\
8,917.70 \\
8,891.06 \\
84.24 \\
79.56\end{array}$ & $\begin{array}{l}1 \\
1 \mathrm{~h} \\
1 \\
2 \\
3\end{array}$ & & $\begin{array}{l}172.68 \\
210.58 \\
244.17 \\
252.80 \\
258.73\end{array}$ & $a^{2} \mathrm{P}_{11 / 2}-x^{2} \mathrm{~F}_{21 / 2}^{\circ}$ & & \\
\hline $\begin{array}{l}75.05 \\
71.00 \\
67.35 \\
39.64 \\
25.86\end{array}$ & $\begin{array}{r}2 \\
4 \\
3 \\
20 \\
50\end{array}$ & & $\begin{array}{l}264.45 \\
269.60 \\
274.24 \\
309.57 \\
327.23\end{array}$ & $\begin{array}{l}a^{4} \mathrm{~F}_{31 / 3}-z^{2} \mathrm{D}_{21 / 3}^{\circ} \\
a^{2} \mathrm{~F}_{31 / 2}-y^{2} \mathrm{D}_{21 / 2}^{\circ}\end{array}$ & & \\
\hline
\end{tabular}


TABLE 6.-The arc spectrum of lanthanum (La I)-Continued

\begin{tabular}{|c|c|c|c|c|c|c|}
\hline \multirow{2}{*}{$\lambda_{\text {air I. }}$ A. } & \multicolumn{2}{|c|}{$\begin{array}{l}\text { Intensities, tem } \\
\text { perature class }\end{array}$} & \multirow{2}{*}{$\nu_{\nabla 20} \mathrm{~cm}^{-1}$} & \multirow{2}{*}{$\begin{array}{l}\text { Term combi- } \\
\text { nation }\end{array}$} & \multicolumn{2}{|c|}{ Zeeman effects } \\
\hline & B. S. & K. \& C. & & & Observed & Computed \\
\hline $\begin{array}{r}21.66 \\
8,818.96 \\
8,797.6 \\
72.02 \\
67.92\end{array}$ & $\begin{array}{cc}1 & \mathrm{~h} \\
20 & \\
2 & \mathrm{~h} \\
1 \\
4\end{array}$ & & $\begin{array}{l}332.62 \\
336.10 \\
363.6 \\
396.76 \\
402.07\end{array}$ & & & - \\
\hline $\begin{array}{c}60.4 \\
48.42 \\
24.12 \\
20.42 \\
8,703.13\end{array}$ & $\begin{array}{r}1 \\
50 \\
1 \\
20 \\
5\end{array}$ & & $\begin{array}{l}411.9 \\
427.50 \\
459.33 \\
464.19 \\
486.97\end{array}$ & $\begin{array}{l}a^{4} \mathrm{~F}_{11 / 2}-z^{4} \mathrm{D}_{01 / 2}^{\circ} \\
a^{2} \mathrm{G}_{41 / 2}-y^{4} \mathrm{P}_{41 / 2}^{\circ} \\
a^{2} \mathrm{G}_{31 / 2}-z^{2} \mathrm{G}_{31 / 2}^{0}\end{array}$ & & 1 \\
\hline $\begin{array}{c}8,674.40 \\
72.10 \\
38.4 \\
24.22 \\
8,621.55\end{array}$ & $\begin{array}{r}60 \\
30 \\
10 \\
6 \\
2\end{array}$ & & $\begin{array}{l}525.01 \\
528.07 \\
573.0 \\
592.07 \\
595.66\end{array}$ & $\begin{array}{l}a^{4} \mathrm{~F}_{31 / 2}-z^{4} \mathrm{~F}_{31 / 2} \\
a^{2} \mathrm{G}_{41 / 2}-z^{2} \mathrm{G}_{31 / 2}^{3} \\
b^{2} \mathrm{D}_{11 / 2}-y^{2} \mathrm{P}_{11 / 2}^{0} \\
a^{2} \mathrm{~F}_{31 / 2}-z^{4} \mathrm{G}_{31 / 2}^{0}\end{array}$ & & 1 \\
\hline $\begin{array}{c}8,590.97 \\
58.9 \\
45.44 \\
43.46 \\
29.68\end{array}$ & $\begin{array}{r}6 \\
1 \\
50 \\
20 \\
3\end{array}$ & & $\begin{array}{l}636.94 \\
680.5 \\
698.94 \\
701.65 \\
720.55\end{array}$ & $\begin{array}{l}b^{2} \mathrm{D}_{11 / 2}-y^{4} \mathrm{~F}_{11 / 2}^{\circ} \\
y^{2} \mathrm{D}_{21 / 2}^{0}-e^{4} \mathrm{~F}_{31 / 2} \\
a^{4} \mathrm{~F}_{21 / 2}-z^{4} \mathrm{D}_{11 / 2}^{\circ} \\
a^{2} \mathrm{G}_{31 / 2}-x^{2} \mathrm{~F}_{31 / 2}^{\circ}\end{array}$ & & \\
\hline $\begin{array}{r}13.55 \\
8,507.37 \\
8,476.48 \\
67.62 \\
8,440.06\end{array}$ & $\begin{array}{r}15 \\
10 \\
30 \\
15 \\
3\end{array}$ & t & $\begin{array}{r}742.76 \\
751.29 \\
794.12 \\
806.45 \\
845.01\end{array}$ & $\begin{array}{l}a^{2} \mathrm{G}_{41 / 2}-x^{2} \mathrm{~F}_{31 / 2}^{\circ} \\
b^{2} \mathrm{D}_{11 / 2}-y^{2} \mathrm{P}_{01 / 2}^{0} \\
a^{4} \mathrm{~F}_{21 / 3}-z^{2} \mathrm{D}_{21 / 2}^{01} \\
z^{4} \mathrm{G}_{51 / 2}^{0}-e^{4} \mathrm{~F}_{41 / 2}\end{array}$ & & 1 \\
\hline $\begin{array}{r}8,379.80 \\
58.50 \\
46.60 \\
34.44 \\
24.72\end{array}$ & $\begin{array}{c}20 \\
2 \mathrm{~h} \\
100 \\
3 \\
100\end{array}$ & $\begin{array}{l}12, \text { III } \\
15, \text { III }\end{array}$ & $\begin{array}{r}930.18 \\
960.59 \\
977.64 \\
11,995.11 \\
12,009.12\end{array}$ & $\begin{array}{l}z^{4} \mathrm{G}_{41 / 2}^{0}-e^{4} \mathrm{~F}_{31 / 2}^{0} \\
a^{4} \mathrm{~F}_{41 / 2}-z^{4} \mathrm{D}_{31 / 2}^{0} \\
a^{4} \mathrm{~F}_{31 / 2}-z^{4} \mathrm{D}_{21 / 2}^{0}\end{array}$ & & \\
\hline $\begin{array}{r}24.59 \\
16.05 \\
8,302.82 \\
8,247.46 \\
37.90\end{array}$ & $\begin{array}{c}\text { HNR } \\
10 \\
4 \\
60 \\
3\end{array}$ & $\begin{array}{l}\text { 1, III A } \\
2, \text { IV A } \\
10, \text { III }\end{array}$ & $\begin{array}{l}009.30 \\
021.64 \\
040.80 \\
121.62 \\
135.68\end{array}$ & $\begin{array}{l}a^{4} \mathrm{~F}_{21 / 2}-z^{4} \mathrm{~F}_{31 / 2}^{0} \\
a^{4} \mathrm{~F}_{21 / 2}-z^{2} \mathrm{D}_{11 / 2}^{0} \\
a^{4} \mathrm{~F}_{11 / 2}-z^{4} \mathrm{D}_{11 / 2}^{0} \\
a^{4} \mathrm{~F}_{413 / 2}-z^{4} \mathrm{~F}_{41 / 2}^{011} \\
a^{4} \mathrm{~F}_{11 / 2}-z^{2} D_{21 / 2}^{0}\end{array}$ & & \\
\hline $\begin{array}{r}11.65 \\
8,203.38 \\
8,086.10 \\
84.53 \\
51.38\end{array}$ & $\begin{array}{c}2 \\
3 \\
20+\mathrm{M} \\
3 \\
10\end{array}$ & $\begin{array}{l}15, \text { III } \\
10, \text { III }\end{array}$ & $\begin{array}{l}174.48 \\
186.75 \\
363.50 \\
365.92 \\
416.82\end{array}$ & $\begin{array}{l}a^{4} \mathrm{~F}_{21 / 2}-z^{2} \mathrm{~F}_{21 / 2}^{\circ} \\
a^{4} \mathrm{~F}_{11 / 2}-z^{2} \mathrm{D}_{11 / 2}^{0} \\
a^{2} \mathrm{G}_{41 / 2}-z^{2} \mathrm{G}_{41 / 2}^{0} \\
a^{4} \mathrm{~F}_{41 / 2}-z^{2} \mathrm{~F}_{31 / 2}^{0}\end{array}$ & & \\
\hline $\begin{array}{r}8.001 .91 \\
7,964.86 \\
48.30 \\
7,931.18 \\
7,864.98\end{array}$ & $\begin{array}{c}4 \\
5 \\
\text { HNR } \\
1 \\
1\end{array}$ & $\begin{array}{l}4, \text { III A } \\
6, \text { III } \\
10, \text { III }\end{array}$ & $\begin{array}{l}493.58 \\
551.70 \\
577.85 \\
605.00 \\
711.10\end{array}$ & $\begin{array}{l}a^{4} \mathrm{~F}_{21 / 2}-z^{4} \mathrm{D}_{21 / 2}^{0} \\
a^{4} \mathrm{~F}_{11 / 2}-z^{2} \mathrm{P}_{01 / 2}^{\circ} \\
a^{2} \mathrm{D}_{21 / 2}-z^{4} \mathrm{~F}_{21 / 2}^{\circ} \\
a^{4} \mathrm{~F}_{33 / 2}-z^{4} \mathrm{D}_{31 / 2}^{\circ} \\
a^{2} \mathrm{~F}_{31 / 2}-y^{4} \mathrm{~F}_{31 / 2}^{\circ}\end{array}$ & & \\
\hline $\begin{array}{r}7,841.76 \\
7,737.74 \\
7,664.38 \\
7,539.24 \\
33.64\end{array}$ & $\begin{array}{c}3 \\
1 \mathrm{~h} \\
4 \\
10 \\
2\end{array}$ & $\begin{array}{l}? \text { III } \\
15, \text { II }\end{array}$ & $\begin{array}{r}748.74 \\
12,920.12 \\
13,043.78 \\
260.29 \\
.270 .14\end{array}$ & $\begin{array}{l}a^{4} \mathrm{~F}_{31 / 2}-z^{4} \mathrm{~F}_{41 / 2}^{0} \\
a^{2} \mathrm{~F}_{31 / 2}-x^{2} \mathrm{~F}_{21 / 2}^{0} \\
a^{4} \mathrm{~F}_{31 / 2}-z^{2} \mathrm{~F}_{31 / 2}^{0} \\
a^{2} \mathrm{D}_{11 / 2}-z^{4} \mathrm{~F}_{11 / 2}^{0} \\
a^{4} \mathrm{~F}_{21 / 2}-z^{2} \mathrm{P}_{11 / 2}\end{array}$ & & \\
\hline \begin{tabular}{r|}
$7,501.78$ \\
$7,498.82$ \\
$7,463.08$ \\
$7,382.73$ \\
79.71
\end{tabular} & $\begin{array}{l}1 \\
2 \\
5 \\
6\end{array}$ & $\begin{array}{l}5, \text { III A } \\
10, \text { III } \\
5, \text { III A } \\
10, \text { III A }\end{array}$ & $\begin{array}{l}326.50 \\
331.76 \\
395.61 \\
541.40 \\
546.94\end{array}$ & $\begin{array}{l}a^{2} \mathrm{~F}_{21 / 2}-y^{4} \mathrm{~F}_{21 / 2}^{0} \\
a^{2} \mathrm{~F}_{31 / 2}-y^{4} \mathrm{~F}_{41 / 2}^{0} \\
a^{2} \mathrm{~F}_{31 / 2}-z^{2} \mathrm{G}_{31 / 2}^{0} \\
a^{2} \mathrm{P}_{11 / 2}-z^{2} \mathrm{~S}^{0} 1 / 2 \\
a^{2} \mathrm{G}_{41 / 2}-y^{2} \mathrm{G}_{41 / 2}^{8}\end{array}$ & 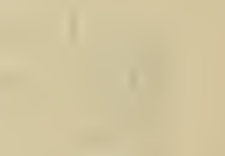 & 1 \\
\hline $\begin{array}{r}45.36 \\
44.42 \\
34.18 \\
20.90 \\
7,308.46\end{array}$ & $\begin{array}{c}25 \\
\text { HNR } \\
50 \\
2 \\
2 \mathrm{~h}\end{array}$ & $\begin{array}{l}20, \text { III A } \\
2 \text {, III A } \\
60, \text { II }\end{array}$ & $\begin{array}{l}610.30 \\
612.03 \\
631.04 \\
655.77 \\
679.01\end{array}$ & $\begin{array}{c}a^{2} \mathrm{~F}_{31 / 2}-x^{2} \mathrm{~F}_{31 / 2}^{\circ} \\
a^{4} \mathrm{~F}_{11 / 2}-z^{2} \mathrm{P}_{1 / 2}^{\circ} \\
a^{2} \mathrm{D}_{1 / 1 / 2}-z^{4} \mathrm{~F}_{21 / 2}^{\circ} \\
a^{2} \mathrm{D}_{21 / 2}-z^{4} \mathrm{D}_{21 / 2}^{01} \\
a^{2} \mathrm{~S}_{1 / 2}-{ }^{4} \mathrm{~S}_{11 / 2}^{\circ}\end{array}$ & $\begin{array}{l}(0.42) 1.18 ? \\
(0.00 \mathrm{w}) 1.30 \mathrm{w}\end{array}$ & $\begin{array}{l}(0.39) 1.06 \\
(0.00) 1.30\end{array}$ \\
\hline $\begin{array}{r}7,285.83 \\
70.30 \\
70.07 \\
63.68 \\
62.83\end{array}$ & $\begin{array}{c}1 \\
\text { HNR } \\
\text { HNR } \\
2 \\
2\end{array}$ & $\begin{array}{c}5, \text { II A } \\
10, \text { III }\end{array}$ & $\begin{array}{l}721.50 \\
750.81 \\
751.24 \\
763.34 \\
764.95\end{array}$ & $\begin{array}{l}a^{2} \mathrm{D}_{21 / 2}-z^{2} \mathrm{D}_{21 / 2}^{\circ} \\
a^{2} \mathrm{~F}_{21 / 2}-y^{4} \mathrm{~F}_{31 / 2}^{\circ}\end{array}$ & & \\
\hline
\end{tabular}


TABLE 6.-The arc spectrum of lanthanum ( $\mathrm{La} I$ ) - Continued

\begin{tabular}{|c|c|c|c|c|c|c|}
\hline \multirow{2}{*}{$\lambda_{\text {air I. A. }}$} & \multicolumn{2}{|c|}{$\begin{array}{l}\text { Intensities, tem- } \\
\text { perature class }\end{array}$} & \multirow{2}{*}{$\nu$ vso cm-1 } & \multirow{2}{*}{$\begin{array}{l}\text { Term combi- } \\
\text { nation }\end{array}$} & \multicolumn{2}{|c|}{ Zeeman effects } \\
\hline & B. S. & K. \& C. & & & Observed & Computed \\
\hline $\begin{array}{r}50.38 \\
19.92 \\
7,217.16 \\
7,161.25 \\
58.11\end{array}$ & $\begin{array}{r}1 \\
15 \\
2 \\
40 \\
30\end{array}$ & $\begin{array}{l}15, \text { II A } \\
50, \text { III } \\
50, \text { III A }\end{array}$ & $\begin{array}{l}788.59 \\
846.76 \\
852.06 \\
960.20 \\
966.32\end{array}$ & $\begin{array}{l}a^{4} \mathrm{~F}_{41 / 2}-y^{2} \mathrm{~F}_{31 / 2}^{\circ} \\
a^{4} \mathrm{~F}_{21 / 2}-y^{2} \mathrm{~F}_{21 / 2}^{\circ} \\
a^{2} \mathrm{~F}_{21 / 2}-x^{2} \mathrm{~F}_{21 / 2}^{\circ} \\
a^{2} \mathrm{D}_{21 / 2}-z^{4} \mathrm{~F}_{31 / 2}^{\circ}\end{array}$ & $\begin{array}{l}(0.00) 0.91 \\
(0.00) 1.27\end{array}$ & $\begin{array}{l}(0.00) 0.90 \\
(0.00) 1.22\end{array}$ \\
\hline $\begin{array}{r}51.92 \\
7,149.76 \\
7,098.18 \\
92.40 \\
76.36\end{array}$ & \begin{tabular}{|c|}
$\mathrm{HNR}$ \\
2 \\
2 \\
$\mathrm{HNR}$ \\
3
\end{tabular} & $\begin{array}{l}\text { 1, III A } \\
2, \text { III } \\
2, \text { I A } \\
3, \text { III }\end{array}$ & $\begin{array}{r}978.42 \\
13,982.63 \\
14,084.24 \\
095.72 \\
127.67\end{array}$ & $\left\{\begin{array}{l}a^{2} \mathrm{D}_{21 / 2}-z^{2} \mathrm{D}_{11 / 2}^{\circ} \\
a^{4} \mathrm{P}_{21 / 2}-x^{2} \mathrm{~F}_{31 / 2}^{0} \\
a^{2} \mathrm{D}_{1 / 2}-z^{4} \mathrm{D}_{11 / 2}\end{array}\right.$ & & \\
\hline $\begin{array}{l}68.37 \\
62.4 \\
45.96\end{array}$ & $\begin{array}{c}60 \\
1 \mathrm{~h} \\
200\end{array}$ & $\begin{array}{l}100, \mathrm{II} \\
300, \mathrm{II}\end{array}$ & $\begin{array}{l}143.64 \\
155.6 \\
188.62\end{array}$ & $\begin{array}{l}a^{2} \mathrm{D}_{21 / 2}-z^{2} \mathrm{~F}_{23 / 2}^{0} \\
a^{2} \mathrm{P}_{13 / 2}-w^{2} \mathrm{~F}_{21 / 2}^{2} \\
a^{4} \mathrm{~F}_{11 / 2}-y^{2} \mathrm{~F}_{221 / 2}\end{array}$ & $\begin{array}{l}(0.69) ? \\
(0.21,0.65) 1.04,1.47\end{array}$ & $\begin{array}{l}(0.66) 1.05 \\
(0.21,0.62) 0.20,0.63\end{array}$ \\
\hline $\begin{array}{l}32.07 \\
23.67\end{array}$ & $\begin{array}{r}25 \\
150\end{array}$ & $\begin{array}{l}\text { 15, III } \\
150, \text { III }\end{array}$ & $\begin{array}{l}216.65 \\
233.66\end{array}$ & $\begin{array}{l}a^{2} \mathrm{P}_{1 / 2}-z^{2} \mathrm{~S}_{01 / 2}^{0} \\
a^{2} F_{31 / 2}-z^{2} G_{41 / 2}^{6}\end{array}$ & $\begin{array}{l}(0.60) 1.27 \\
(0.00) 1.16\end{array}$ & $\begin{array}{l}(0.60) 1.27 \\
(0.00) 1.16\end{array}$ \\
\hline $\begin{array}{r}7,013.15 \\
6,978.09 \\
76.87 \\
35.03 \\
25.27\end{array}$ & $\begin{array}{r}2 \\
2 \\
2 \\
50 \\
80\end{array}$ & $\begin{array}{r}3, \text { III } \\
50, \text { III } \\
100, \text { III }\end{array}$ & $\begin{array}{l}255.00 \\
326.62 \\
329.13 \\
415.58 \\
435.90\end{array}$ & $\begin{array}{l}z^{4} \mathrm{D}_{31 / 2}^{0}-e^{4} \mathrm{~F}_{21 / 2} \\
a^{2} \mathrm{P}_{11 / 2}-x^{4} \mathrm{D}_{21 / 2} \\
\\
a^{4} \mathrm{~F}_{31 / 2}-y^{2} \mathrm{~F}_{31 / 2}^{\circ} \\
a^{2} \mathrm{~F}_{21 / 2}-z^{2} \mathrm{G}_{31 / 2}^{3}\end{array}$ & $\begin{array}{l}(0.48) 1.14 \\
(0.00 \mathrm{~W}) 1.58 \mathrm{~A}^{2}\end{array}$ & $\begin{array}{l}(0.41) 1.15 \\
(0.00) 1.53 \mathrm{~A}^{3}\end{array}$ \\
\hline $\begin{array}{r}18.33 \\
17.26 \\
03.08 \\
6,902.08 \\
6,889.63\end{array}$ & $\begin{array}{r}8 \\
10 \\
2 \\
3 \\
2 \mathrm{~h}\end{array}$ & $\begin{array}{l}\text { 8, III A } \\
10, \text { III A } \\
1 \text {, III A } \\
\text { 1, III } \\
\text { tr, V }\end{array}$ & $\begin{array}{l}450.38 \\
452.61 \\
482.30 \\
484.40 \\
489.54\end{array}$ & $\begin{array}{l}a^{2} \mathrm{D}_{21 / 3}-z^{4} \mathrm{D}_{31 / 2}^{0} \\
a^{4} \mathrm{~F}_{31 / 2}-z^{4} \mathrm{G}_{21 / 2}^{0} \\
a^{4} \mathrm{~F}_{41 / 2}-z^{4} G_{31 / 2}^{0} \\
a^{2} \mathrm{P}_{1 / 2}-x^{4} D_{01 / 2}^{0} \\
a^{2} G_{41 / 2}-w^{2} F_{31 / 2}^{0}\end{array}$ & & \\
\hline $\begin{array}{r}98.41 \\
23.80 \\
21.51 \\
19.14 \\
6,816.29\end{array}$ & $\begin{array}{l}40 \\
5 \mathrm{~d} \\
1\end{array}$ & $\begin{array}{l}\text { tr, V } \\
15, \text { II A } \\
3 n, V \\
1 n, V ?\end{array}$ & $\begin{array}{l}492.10 \\
650.56 \\
655.47 \\
660.57 \\
666.70\end{array}$ & $a^{2} \mathrm{~F}_{21 / 2}-x^{2} \mathrm{~F}_{3 / 2}^{0}-x^{4} \mathrm{D}_{1 / 2}^{\circ}$ & $(0.00) 1.15$ & $(0.00) 1.12$ \\
\hline $\begin{array}{r}6,796.73 \\
83.55 \\
78.19 \\
76.69 \\
60.73\end{array}$ & $\begin{array}{c}4 \\
1 \\
1 \\
1 \\
\text { HNR }\end{array}$ & $\begin{array}{l}\text { 4, II A } \\
\text { tr, IV A } \\
\text { tr, V } \\
\text { 1, III A } \\
\text { 1, III A }\end{array}$ & $\begin{array}{l}708.90 \\
737.48 \\
749.14 \\
752.40 \\
787.23\end{array}$ & $\begin{array}{l}a^{2} \mathrm{D}_{1 / 2}-z^{4} \mathrm{D}_{11 / 2}^{\circ} \\
z^{2} \mathrm{~F}_{31 / 2}^{\circ}-e^{4} \mathrm{D}_{31 / 2} \\
z^{4} \mathrm{O}_{11 / 2}-33 \\
a^{4} \mathrm{~F}_{21 / 2}-z^{4} \mathrm{P}_{11 / 2}^{\circ}\end{array}$ & & 1 \\
\hline $\begin{array}{l}60.23 \\
53.05 \\
48.12 \\
41.20 \\
37.29\end{array}$ & $\begin{array}{r}1 \\
40 \\
10 \\
2 \\
1\end{array}$ & $\begin{array}{l}50, \text { I A } \\
10, \text { II A }\end{array}$ & $\begin{array}{l}788.32 \\
804.04 \\
814.86 \\
830.07 \\
838.67\end{array}$ & $\begin{array}{l}a^{2} \mathrm{P}_{11 / 2}-v^{2} \mathrm{P}_{21 / 2}^{\circ} \\
a^{2} \mathrm{D}_{11 / 2}-z^{2} \mathrm{D}_{21 / 2}^{\circ} \\
b^{2} \mathrm{D}_{11 / 2}-z^{2} \mathrm{~S}_{01 / 2}^{0}\end{array}$ & $(0.00 \mathrm{w}) 1.51 \mathrm{~A}^{2}$ & $(0.00) 1.51 \mathrm{~A}^{2}$ \\
\hline $\begin{array}{r}15.96 \\
6,709.49 \\
6,699.86 \\
99.26 \\
92.86\end{array}$ & $\begin{array}{r}150 \\
3 \\
2 \\
20\end{array}$ & $\begin{array}{l}1, \text { IVA? } \\
200, \text { I } \\
4, \text { III A } \\
2, \text { III? } \\
30, \text { I A }\end{array}$ & $\begin{array}{r}885.80 \\
900.16 \\
921.57 \\
922.91 \\
14,937.18\end{array}$ & $\begin{array}{l}z^{4} \mathrm{D}_{1 / 2}^{\circ}-34^{4} \\
a^{4} \mathrm{~F}_{21 / 2}-y^{2} \mathrm{~F}_{21 / 2}^{\circ} \\
a^{4} \mathrm{~F}_{21 / 2}-z^{4} \mathrm{G}_{21 / 2}^{\circ}\end{array}$ & $\begin{array}{l}(0.00) 1.18 \\
(0.00) 1.06\end{array}$ & $\begin{array}{l}(0.00) 1.14 \\
(0.09) 1.05\end{array}$ \\
\hline $\begin{array}{l}64.45 \\
61.40 \\
58.06 \\
50.81 \\
45.15\end{array}$ & $\begin{array}{r}1 \\
60 \\
1 \\
80 \\
7\end{array}$ & $\begin{array}{l}80, \text { I A } \\
100, \text { I A } \\
8, \text { IV }\end{array}$ & $\begin{array}{r}15,000.85 \\
007.72 \\
015.25 \\
031.62 \\
044.42\end{array}$ & $\begin{array}{r}a^{4} \mathrm{~F}_{41 / 2}-z^{4} \mathrm{G}_{i 1 / 2}^{0} \\
a^{4} \mathrm{P}_{1 / 2}-y^{4} \mathrm{D}_{0}^{1 / 2} \\
a^{2} \mathrm{D}_{11 / 2}-z^{2} \mathrm{D}_{11 / 2} \\
z^{4} \mathrm{~F}_{41 / 2}^{112-e^{4} \mathrm{D}_{31 / 2}}\end{array}$ & $\begin{array}{l}(0.65) 1.23 \mathrm{~B} ? \\
(0.00 \mathrm{w}) 0.88\end{array}$ & $\begin{array}{l}(0.66) 1.24 \\
(0.25) 0.88\end{array}$ \\
\hline $\begin{array}{l}44.40 \\
31.20 \\
28.4 \\
16.59 \\
12.48\end{array}$ & $\begin{array}{r}30 \\
3 \\
1 \\
60 \\
3\end{array}$ & $\begin{array}{l}\text { 40, I A } \\
3, \text { V? } \\
1, \text { IV A } \\
80, \text { I }\end{array}$ & $\begin{array}{l}046.12 \\
076.07 \\
082.4 \\
109.36 \\
118.75\end{array}$ & $\begin{array}{l}a^{2} \mathrm{D}_{21 / 2}-z^{4} \mathrm{D}_{31 / 2}^{0} \\
b^{2} \mathrm{D}_{11 / 2}-x^{4} \mathrm{D}_{01 / 2}^{\circ} \\
a^{4} \mathrm{~F}_{31 / 2}-z^{4} \mathrm{G}_{31 / 2}^{\circ}\end{array}$ & $(0.59) 1.12 \mathrm{~B}$ & $(0.47) 1.14$ \\
\hline $\begin{array}{r}08.25 \\
07.7 \\
6,600.17 \\
6,599.48 \\
93.45\end{array}$ & $\begin{array}{r}40 \\
1 \\
25 \\
40\end{array}$ & $\begin{array}{l}40, \text { II } \\
3, \text { IV } \\
50, \text { II A } \\
\text { tr, IV A } \\
60, \text { I }\end{array}$ & $\begin{array}{l}128.43 \\
129.7 \\
146.95 \\
148.53 \\
162.39\end{array}$ & $\begin{array}{c}a^{2} \mathrm{G}_{31 / 2}-z^{2} \mathrm{H}_{11 / 2}^{\circ} \\
a^{2} \mathrm{P}_{1 / 2}-4_{1}^{1} 1 / 2 \\
a^{4} \mathrm{~F}_{21 / 2}-z^{4} \mathrm{P}_{21 / 2}^{\circ} \\
a^{4} \mathrm{~F}_{21 / 2}-y^{2} \mathrm{D}_{11 / 2}^{\circ}\end{array}$ & $\begin{array}{l}(0.00) 1.01 \\
(0.35) 1.12 \\
(0.60 \mathrm{w}) 1.30 \mathrm{~A}^{2}\end{array}$ & $\begin{array}{l}(0.00) 1.00 \\
(0.37) 1.12 \\
(0.00) 1.33 \mathrm{~A}^{2}\end{array}$ \\
\hline $\begin{array}{l}90.59 \\
82.18 \\
78.51 \\
68.54 \\
65.45\end{array}$ & $\begin{array}{r}1 \\
4 \\
200 \\
1 \\
40\end{array}$ & $\begin{array}{r}6, \text { IV } \\
400, \text { I } \\
40, \text { I A }\end{array}$ & $\begin{array}{l}168.97 \\
188.35 \\
196.82 \\
219.89 \\
227.05\end{array}$ & $\begin{array}{l}a^{2} \mathrm{~F}_{31 / 2}-1_{31 / 2}^{\circ} \\
z^{4} \mathrm{D}_{31 / 2}^{0}-e^{4} \mathrm{D}_{31 / 2} \\
a^{2} \mathrm{D}_{11 / 2}-z^{2} \mathrm{~F}_{21 / 2}^{\circ} \\
a^{2} \mathrm{D}_{11 / 2}-z^{2} \mathrm{P}^{0} 1 / 1 / 2 \\
a^{2} \mathrm{D}_{21 / 2}-z^{2} \mathrm{P}_{11 / 2}^{0}\end{array}$ & $\begin{array}{l}(0.00 \mathrm{~h}) 1.41 \\
(0.00) 1.00\end{array}$ & $(0.00) 0.98$ \\
\hline
\end{tabular}


TABLE 6.-The arc spectrum of lanthanum ( $L a I)$-Continued

\begin{tabular}{|c|c|c|c|c|c|c|}
\hline \multirow{2}{*}{$\lambda_{\text {air I. A. }}$} & \multicolumn{2}{|c|}{$\begin{array}{l}\text { Intensities, tem- } \\
\text { perature class }\end{array}$} & \multirow{2}{*}{$\nu_{\mathrm{vac}} \mathrm{Cmi}^{-1}$} & \multirow{2}{*}{$\begin{array}{l}\text { Term combi- } \\
\text { nation }\end{array}$} & \multicolumn{2}{|c|}{ Zeeman effects } \\
\hline & B. S. & K. \& C. & & & Obserred & Computed \\
\hline $\begin{array}{l}55.95 \\
55.11 \\
51.78 \\
49.16 \\
43.17\end{array}$ & $\begin{array}{r}2 \\
1 \\
2 \\
300\end{array}$ & 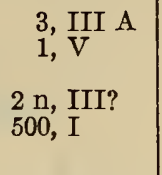 & $\begin{array}{l}249.12 \\
251.07 \\
258.82 \\
264.92 \\
278.90\end{array}$ & $\begin{array}{l}a^{2} \mathrm{~F}_{31 / 2}-y^{4} \mathrm{D}_{31 / 2} \\
b^{2} \mathrm{D}_{11 / 2}-x^{4} \mathrm{D}_{11 / 2}^{\circ} \\
a^{2} \mathrm{P}_{13 / 2}-x^{4} \mathrm{~F}_{21 / 2}^{\circ} \\
a^{4} \mathrm{~F}_{13 / 2}-z^{4} \mathrm{G}_{21 / 2}^{\circ}\end{array}$ & $\begin{array}{l}(0.33,0.99) 0.00,0.74, \\
\quad 1.41,2.07\end{array}$ & $\begin{array}{l}(0.33,0.99) 0.08,0.75 \\
\quad 1.40,2.06\end{array}$ \\
\hline $\begin{array}{r}23.86 \\
20.70 \\
19.30 \\
17.35 \\
6,506.25\end{array}$ & $\begin{array}{r}2 \\
15 \\
3 \\
1 \\
4\end{array}$ & $\begin{array}{l}2, \text { III A? } \\
20, \text { IV } \\
4, \text { IV } \\
6, \text { IV }\end{array}$ & $\begin{array}{l}324.12 \\
331.55 \\
334.84 \\
339.43 \\
365.59\end{array}$ & $\begin{array}{l}b^{2} \mathrm{D}_{23 / 2}-v^{2} \mathrm{~F}_{21 / 2}^{\circ} \\
z^{4} \mathrm{~F}_{31 / 2}^{\circ}-e^{4} \mathrm{~F}_{21 / 2} \\
z^{4} \mathrm{G}_{21 / 3}^{\circ}-35 \\
z^{4} \mathrm{D}_{3 / 2}-33\end{array}$ & $(0.00 \mathrm{~h}) 1.10 \mathrm{~h}$ & \\
\hline $\begin{array}{r}6,492.86 \\
85.54 \\
80.20 \\
68.44 \\
55.99\end{array}$ & $\begin{array}{r}4 \\
20 \\
1 \\
10 \\
250\end{array}$ & $\begin{array}{l}5, \mathrm{~V} \\
20, \mathrm{II} A \\
8, \text { II A } \\
300, \mathrm{I}\end{array}$ & $\begin{array}{l}397.29 \\
414.66 \\
427.37 \\
455.42 \\
485.22\end{array}$ & $\begin{array}{l}a^{2} \mathrm{~F}_{31 / 3}-2 i \frac{1}{2} \\
a^{2} \mathrm{~F}_{21 / 2}-y^{4} \mathrm{D}_{11 / 2} \\
b^{2} \mathrm{D}_{21 / 2}-z^{4} \mathrm{~S}_{1 / 2} \\
a^{2} \mathrm{D}_{21 / 2}-z^{2} \mathrm{~F}_{31 / 2}^{\circ}\end{array}$ & $\begin{array}{l}(0.00) 1.10 \\
(0.00) 1.18\end{array}$ & $\begin{array}{l}(0.00) 1.10 \\
(0.00) 1.15\end{array}$ \\
\hline 54.50 & 150 & $200, \mathrm{I}$ & 488.80 & $a^{4} \mathrm{~F}_{11 / 2}-z^{4} \mathrm{P}_{21 / 2}^{0}$ & $(0.38,1.11) 0.80,1.54$, & $(0.39,1.18) \quad 0.02,0.81$, \\
\hline $\begin{array}{l}50.34 \\
49.94 \\
48.25 \\
48.10\end{array}$ & $\begin{array}{r}6 \\
3 \\
4 \\
20\end{array}$ & $\begin{array}{r}8, \text { II A } \\
10 ?, \text { II A } \\
50 ?, \text { II A }\end{array}$ & $\begin{array}{l}498.78 \\
499.74 \\
503.81 \\
504.17\end{array}$ & $\begin{array}{l}a^{2} \mathrm{P}_{11 / 2}-x^{2} \mathrm{D}_{21 / 2}^{\circ} \\
a^{2} \mathrm{D}_{11 / 2}-z^{4} \mathrm{D}_{21 / 2}^{\circ} \\
a^{4} \mathrm{~F}_{11 / 2}-y^{2} \mathrm{D}_{11 / 2}^{1}\end{array}$ & $(0.00) 1.29$ & $(0.00) 1.20$ \\
\hline $\begin{array}{r}26.60 \\
20.90 \\
17.23 \\
6,410.98 \\
6,394.23\end{array}$ & $\begin{array}{r}2 \\
1 \\
2 \\
200 \\
400\end{array}$ & $\begin{array}{l}3, \text { III? A } \\
300, \text { I } \\
600, \text { I }\end{array}$ & $\begin{array}{l}556.04 \\
569.85 \\
578.85 \\
593.94 \\
634.79\end{array}$ & $\begin{array}{l}z^{4} \mathrm{D}_{23 / 2}^{0}-e^{4} \mathrm{~F}_{31 / 2} \\
z^{2} \mathrm{~F}_{31 / 2}^{\circ}-e^{2} \mathrm{~F}_{31 / 2} \\
b^{2} \mathrm{D}_{21 / 2}-x^{2} \mathrm{D}_{11 / 3}^{\mathrm{o}} \\
a^{4} \mathrm{~F}_{21 / 2}-z^{4} \mathrm{G}_{31 / 2}^{0} \\
a^{4} \mathrm{~F}_{33 / 2}-z^{4} \mathrm{G}_{43 / 2}^{4}\end{array}$ & $\begin{array}{l}(0.00) 1.09 \\
(0.00 \mathrm{~W}) 1.02 \mathrm{~A}^{1}\end{array}$ & $\begin{array}{l}(0.00) \\
1.10 \\
(0.00) \\
0.90 \mathrm{~A}^{1}\end{array}$ \\
\hline $\begin{array}{l}80.48 \\
75.50 \\
75.11 \\
60.20 \\
56.38\end{array}$ & $\begin{array}{r}1 \\
2 \\
2 \\
30 \\
3\end{array}$ & $\begin{array}{l}1, \mathrm{~V} \\
2, \mathrm{~V} \\
30, \text { II } \\
4, \text { III A }\end{array}$ & $\begin{array}{l}668.48 \\
680.72 \\
681.68 \\
718.44 \\
727.88\end{array}$ & $\begin{array}{c}z^{4} \mathrm{~F}_{41 / 2}-e^{4} \mathrm{~F}_{41 / 2} \\
a^{2} \mathrm{P}_{1 / 2}-x^{2} \mathrm{D}_{11 / 2}^{\circ} \\
b^{2} \mathrm{D}_{13 / 2}-\mathrm{L}_{11 / 2}\end{array}$ & $(0.00) 0.89$ & $(0.00) 0.93$ \\
\hline $\begin{array}{l}53.63 \\
39.16 \\
33.74 \\
33.24 \\
30.42\end{array}$ & $\begin{array}{l}1 \\
2 \\
3 \\
2 \\
3\end{array}$ & $\begin{array}{l}2, \text { III A } \\
2, \text { IV? } \\
2, \text { III? } \\
2, \text { III A }\end{array}$ & $\begin{array}{l}734.69 \\
770.61 \\
784.10 \\
785.35 \\
792.38\end{array}$ & $\begin{array}{l}a^{2} \mathrm{P}_{13 / 2}-x^{2} \mathrm{P}_{03 / 2}^{0} \\
a^{4} \mathrm{P}_{13 / 2}-z^{2} \mathrm{~S}_{01 / 2}^{0} \\
z^{4} \mathrm{D}_{23 / 2}^{0}-e^{4} \mathrm{D}_{31 / 2} \\
a^{2} \mathrm{~F}_{23 / 2}-y^{4} \mathrm{D}_{21 / 2}^{\circ}\end{array}$ & & \\
\hline 25.90 & 100 & $150, I$ & 803.67 & $a^{2} \mathrm{D}_{21 / 2}-y^{2} \mathrm{~F}_{21 / 2}^{\circ}$ & $\begin{array}{l}(0.63,0.99) 0.25,0.63 \\
\quad 1.01,1.38,1.76\end{array}$ & $\begin{array}{l}(0.19,0.56,0.94) \\
0.64,1.01,1.39,1.77\end{array}$ \\
\hline $\begin{array}{r}18.26 \\
10.13 \\
08.87 \\
6,308.21\end{array}$ & $\begin{array}{l}5 \\
8 \\
2 \\
2\end{array}$ & $\begin{array}{l}12, \text { III A } \\
12, \text { IV } \\
2, \text { III } \\
3, \text { III }\end{array}$ & $\begin{array}{l}822.78 \\
843.16 \\
846.32 \\
847.98\end{array}$ & $a^{2} \mathrm{~F}_{31 / 2}-w^{2} \mathrm{~F}_{21 / 2}^{\circ}$ & & \\
\hline $\begin{array}{r}6,293.57 \\
88.56 \\
87.73 \\
78.31 \\
66.00\end{array}$ & $\begin{array}{r}60 \\
6 \\
7 \\
1 \\
40\end{array}$ & $\begin{array}{l}80, \text { II A } \\
7, \text { III A } \\
8, \text { III A } \\
60, \text { III }\end{array}$ & $\begin{array}{l}884.85 \\
897.50 \\
899.60 \\
923.46 \\
954.74\end{array}$ & $\begin{array}{l}a^{4} \mathrm{~F}_{31 / 2}-y^{2} \mathrm{D}_{21 / 2}^{0} \\
a^{2} \mathrm{P}_{11 / 2}-y^{4} \mathrm{P}_{01 / 3}^{0} \\
b^{2} \mathrm{D}_{21 / 2}-x^{4} \mathrm{D}_{31 / 2}^{3} \\
a^{2} \mathrm{P}_{11 / 2}-y^{4} \mathrm{P}_{1 / 2}^{1 / 2} \\
a^{2} \mathrm{G}_{41 / 2}-z^{2} \mathrm{H}_{31 / 2}^{1}\end{array}$ & $(0.00) 1.06$ & $(0.00) 1.06$ \\
\hline $\begin{array}{l}49.92 \\
38.58 \\
36.76 \\
36.17 \\
34.85\end{array}$ & $\begin{array}{r}300 \\
12 \\
7 \\
8 \\
10\end{array}$ & $\begin{array}{l}500, \text { I } \\
15, \text { III A } \\
10, \text { III A } \\
12, \text { IV } \\
15, \text { III A }\end{array}$ & $\begin{array}{r}15,995.79 \\
16,024.86 \\
029.54 \\
031.06 \\
034.45\end{array}$ & $\begin{array}{c}a^{4} \mathrm{~F}_{41 / 2}-z^{4} \mathrm{G}_{51 / 2}^{\circ} \\
a^{4} \mathrm{P}_{21 / 2}-x^{4} \mathrm{D}_{11 / 2}^{\circ} \\
a^{4} \mathrm{P}_{1 / 2}-z^{2} \mathrm{~S}_{01 / 2}^{1} \\
b^{2} \mathrm{D}_{21 / 2}-x^{2} \mathrm{D}_{21 / 2}^{\circ}\end{array}$ & $(0.00) \quad 1.26$ & $(0.09) \quad 1.27$ \\
\hline $\begin{array}{l}33.51 \\
32.56 \\
25.33 \\
24.24 \\
19.46\end{array}$ & $\begin{array}{l}10 \\
1 \\
2 \mathrm{~h} \\
1 \\
2\end{array}$ & 15, III A & $\begin{array}{l}037.90 \\
040.34 \\
058.97 \\
061.78 \\
074.13\end{array}$ & $\begin{array}{l}a^{4} \mathrm{P}_{11 / 2}-x^{4} \mathrm{D}_{01 / 2}^{\circ} \\
z^{4} \mathrm{~F}_{31 / 2}^{\circ}-e^{4} \mathrm{~F}_{31 / 2} \\
a^{4} \mathrm{P}_{11 / 2}-3_{21 / 2}^{0} \\
b^{2} \mathrm{D}_{11 / 2}-v^{2} \mathrm{~F}_{23 / 2}^{\circ}\end{array}$ & & \\
\hline $\begin{array}{r}18.19 \\
14.35 \\
6,206.76 \\
6,173.74 \\
67.69\end{array}$ & $\begin{array}{l}5 \\
2 \\
2\end{array}$ & $\begin{array}{l}\text { 7, III } \\
1, \text { IV A }\end{array}$ & $\begin{array}{l}077.41 \\
087.34 \\
107.02 \\
193.17 \\
209.05\end{array}$ & $\begin{array}{l}a^{2} \mathrm{G}_{41 / 3}-x^{4} \mathrm{~F}_{41 / 2}^{\circ} \\
z^{2} \mathrm{D}_{11 / 2}^{\circ}-e^{2} \mathrm{~F}_{21 / 2} \\
b^{2} \mathrm{D}_{11 / 2}-z^{4} \mathrm{~S}_{11 / 2}^{\circ} \\
a^{2} \mathrm{~F}_{21 / 2}-1_{31 / 3}^{\circ}\end{array}$ & & \\
\hline
\end{tabular}


TABLE 6.-The arc spectrum of lanthanum ( $L a r$ )-Continued

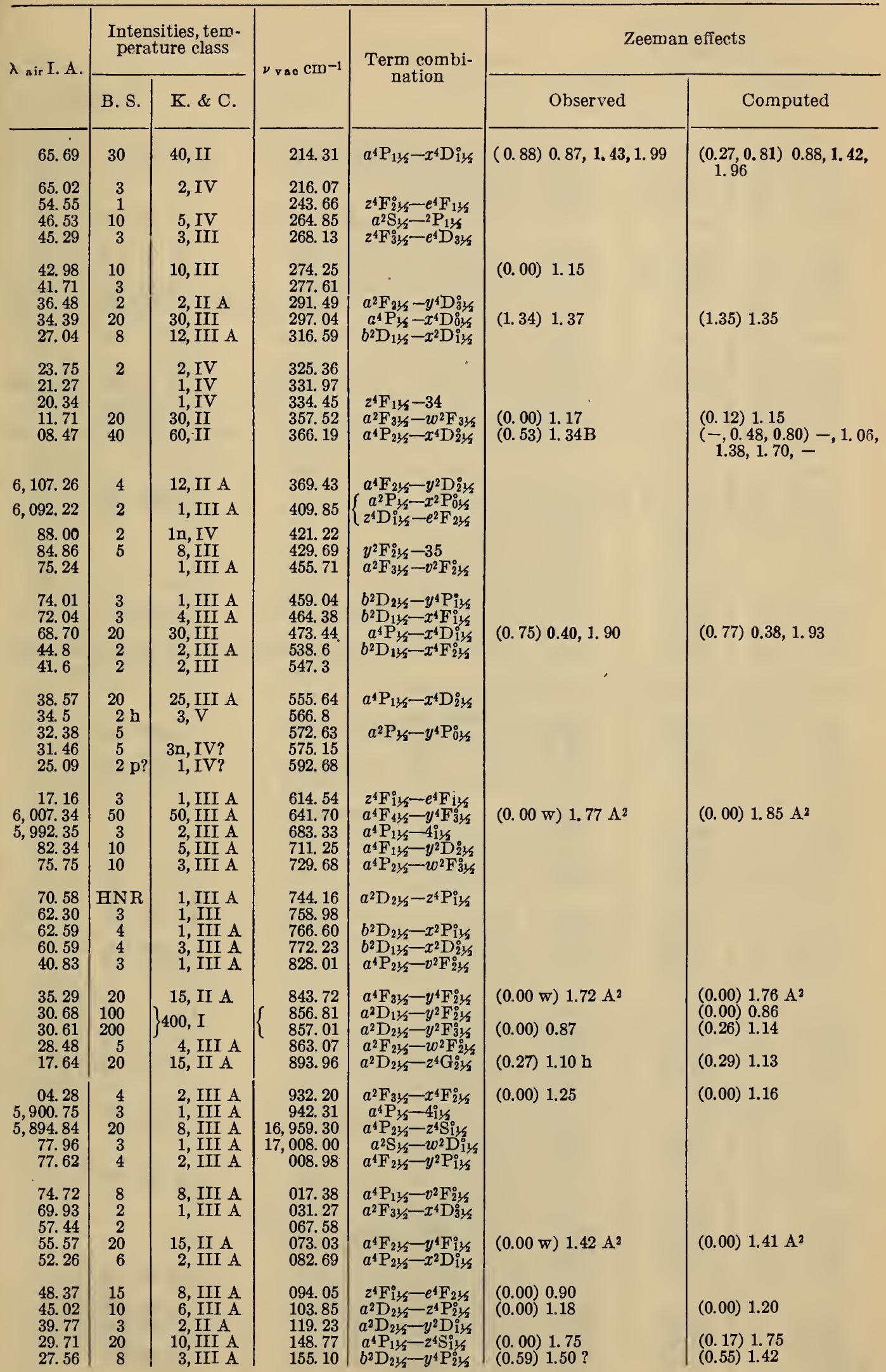


TABLE 6.-The arc spectrum of lanthanum (La I)-Continued

\begin{tabular}{|c|c|c|c|c|c|c|}
\hline \multirow{2}{*}{$\lambda_{\text {air I. A: }}$} & \multicolumn{2}{|c|}{$\begin{array}{l}\text { Intensities, te } n \text { - } \\
\text { perature class }\end{array}$} & \multirow{2}{*}{$\nu_{\nabla a \mathrm{c}} \mathrm{cm}^{-1}$} & \multirow{2}{*}{$\begin{array}{l}\text { Term combi- } \\
\text { nation }\end{array}$} & \multicolumn{2}{|c|}{ Zeeman effects } \\
\hline & B. S. & K. \& C. & & & Observed & Computed \\
\hline $\begin{array}{r}23.82 \\
21.98 \\
13.44 \\
5,802.10 \\
5,791.32\end{array}$ & $\begin{array}{r}15 \\
30 \\
2 \\
2 \\
200\end{array}$ & $\begin{array}{l}\text { 10, III A } \\
30, \text { III } \\
\text { 1, III A } \\
\text { 1, III A } \\
400, \text { I }\end{array}$ & $\begin{array}{l}166.11 \\
171.54 \\
196.76 \\
230.37 \\
262.45\end{array}$ & $\begin{array}{l}a^{2} \mathrm{~F}_{31 / 2}-x^{2} \mathrm{D}_{21 / 3}^{\circ} \\
a^{2} \mathrm{G}_{31 / 2}-y^{2} \mathrm{G}_{31 / 2}^{0} \\
b^{2} \mathrm{D}_{11 / 2}-y^{4} \mathrm{P}_{11 / 2}^{0} \\
a^{4} \mathrm{P}_{21 / 2}^{0}-x^{4} \mathrm{~F}_{1 / 2}^{0} \\
a^{4} \mathrm{~F}_{41 / 2}-y^{4} \mathrm{~F}_{41 / 2}^{0}\end{array}$ & $\begin{array}{l}(0.00 \mathrm{w}) 0.96 \mathrm{~h} \\
(0.00) 0.93 \\
(0.00 \mathrm{w}) 1.31 \mathrm{~B}\end{array}$ & $\begin{array}{l}(0.00) 0.98 \\
(0.12) 0.93 \\
\\
(0.18) 1.30\end{array}$ \\
\hline $\begin{array}{l}89.22 \\
69.97 \\
69.32 \\
61.83\end{array}$ & $\begin{array}{r}150 \\
25 \\
80 \\
50\end{array}$ & $\begin{array}{l}250, \text { I } \\
25, \text { III A } \\
80, \text { I } \\
60, \text { I }\end{array}$ & $\begin{array}{l}268.71 \\
326.32 \\
328.27 \\
350.80\end{array}$ & $\begin{array}{l}a^{4} \mathrm{~F}_{31 / 2}-y^{4} \mathrm{~F}_{31 / 2}^{0} \\
a^{2} \mathrm{~F}_{31 / 2}-v^{2} \mathrm{~F}_{31 / 2}^{3} \\
a^{4} \mathrm{~F}_{21 / 2}-y^{4} \mathrm{~F}_{21 / 2}^{0} \\
a^{4} \mathrm{~F}_{11 / 2}-y^{2} \mathrm{P}_{11 / 2}^{\circ}\end{array}$ & $\begin{array}{l}(0.15) 1.21 \\
(0.00) 1.01 \\
(0.94) 0.00,0.68,1.34\end{array}$ & $\begin{array}{l}(0.12) 1.20 \\
(0.04) 1.02 \\
(0.32,0.96) 0.09,0.73,\end{array}$ \\
\hline 44.41 & 60 & $80, \mathrm{III}$ & 403.41 & $a^{4} \mathrm{P}_{21 / 3}-x^{4} \mathrm{D}_{31 / 2}^{0}$ & $(0.00 \mathrm{w}) 1.24$ & $(0.00) 1.25$ \\
\hline $\begin{array}{l}42.93 \\
40.65\end{array}$ & $\begin{array}{r}4 \\
80\end{array}$ & $\begin{array}{l}2, \text { III A } \\
100, \text { I }\end{array}$ & $\begin{array}{l}407.90 \\
414.81\end{array}$ & $\begin{array}{l}a^{4} \mathrm{P}_{1 / 2}-z^{4} S_{11 / 2}^{\circ} \\
a^{4} \mathrm{~F}_{11 / 2}-y^{4} \mathrm{~F}_{1 / 2}^{11 / 2}\end{array}$ & $(0.47) 0.59 \mathrm{~B}$ & $(0.18,0.55) \quad 0.33,0.60$, \\
\hline $\begin{array}{l}34.93 \\
20.01\end{array}$ & $\begin{array}{r}6 \\
10\end{array}$ & $\begin{array}{r}5, \text { III A } \\
10, \text { III } A\end{array}$ & $\begin{array}{l}432.18 \\
477.65\end{array}$ & $\begin{array}{l}a^{2} \mathrm{G}_{31 / 2}-u^{2} \mathrm{~F}_{21 / 2}^{\circ} \\
a^{1} \mathrm{~F}_{31 / 2}-x^{2} \mathrm{~F}_{21 / 2}^{\circ}\end{array}$ & $\begin{array}{l}(0.00) 0.92 \\
(0.16,0.48,0.81) 1.93 \mathrm{~A}^{2}\end{array}$ & $(0.00) 0.92$ \\
\hline 14.55 & & 1, III A & 494.35 & $a^{2} \mathrm{G}_{31 / 2}-y^{4} \mathrm{G}_{31 / 2}^{0}$ & & \\
\hline $\begin{array}{r}14.01 \\
10.85 \\
03.32 \\
02.57 \\
5,701.15\end{array}$ & $\begin{array}{c}4 \\
2 \mathrm{p} \\
20\end{array}$ & $\begin{array}{l}\text { 5, III A } \\
2, \text { III } \\
10, \text { III } \\
2, \text { III } \\
1, \text { III }\end{array}$ & $\begin{array}{l}496.00 \\
505.68 \\
528.80 \\
531.10 \\
535.47\end{array}$ & $\begin{array}{l}a^{2} \mathrm{~F}_{21 / 2}-v^{2} \mathrm{~F}_{21 / 2}^{0} \\
a^{2} \mathrm{P}_{11 / 2}-w^{2} \mathrm{P}_{11 / 2}^{1} \\
a^{4} \mathrm{~F}_{11 / 2}-y^{2} \mathrm{P}_{11 / 2}^{11 / 2} \\
a^{4} \mathrm{P}_{11 / 2}-x^{2} \mathrm{D}_{11 / 2}^{11 / 2} \\
a^{2} \mathrm{G}_{41 / 2}-y^{4} \mathrm{G}_{31 / 2}^{0}\end{array}$ & $(0.62) ?$ & $(0.66) 1.05$ \\
\hline $\begin{array}{r}5,699.32 \\
96.18 \\
61.34 \\
57.71\end{array}$ & $\begin{array}{r}3 \\
30 \\
2 \\
30\end{array}$ & $\begin{array}{l}5, \text { III } \\
40, \text { I } \\
1, \text { III A } \\
50, \text { II }\end{array}$ & $\begin{array}{l}541.10 \\
550.77 \\
658.78 \\
670.10\end{array}$ & $\begin{array}{l}a^{4} \mathrm{~F}_{41 / 2}-x^{2} \mathrm{~F}_{31 / 2}^{\circ} \\
a^{2} \mathrm{D}_{21 / 2}-z^{4} \mathrm{G}_{31 / 2}^{3} \\
a^{2} \mathrm{G}_{31 / 2}-y^{2} \mathrm{G}_{41 / 2}^{0} \\
a^{4} \mathrm{~F}_{11 / 2}-y^{4} \mathrm{~F}_{21 / 2}^{0}\end{array}$ & $\begin{array}{l}(0.00 \mathrm{w}) 0.80 \mathrm{~A}^{1} \\
(0.29,0.88) 0.73,1.33\end{array}$ & $\begin{array}{l}(0.00) 0.70 \mathrm{~A}^{1} \\
(0.29,0.89) 0.12,0.71 \text {, }\end{array}$ \\
\hline 56.54 & 2 & 1, III A & 673.76 & $a^{2} \mathrm{P}_{11 / 2}-u^{2} \mathrm{~F}_{21 / 2}^{\circ}$ & & \\
\hline $\begin{array}{r}54.8 \\
48.24 \\
39.31 \\
32.02 \\
5,631.22\end{array}$ & $\begin{array}{r}20 \\
50 \\
8 \\
15 \\
60\end{array}$ & $\begin{array}{l}\text { 3, III A } \\
80, \text { III } \\
5 \text {, III A } \\
25, \text { II } \\
100, \text { I }\end{array}$ & $\begin{array}{l}679.2 \\
699.73 \\
727.76 \\
750.70 \\
753.22\end{array}$ & $\begin{array}{l}a^{4} \mathrm{P}_{1 / 2}-x^{4} \mathrm{~F}_{11 / 2}^{\circ} \\
a^{2} \mathrm{G}_{41 / 2}-y^{2} \mathrm{G}_{41 / 2}^{0} \\
a^{4} \mathrm{P}_{11 / 2}-x^{2} \mathrm{D}_{21 / 2}^{01} \\
a^{2} \mathrm{~F}_{21 / 2}-x^{2} \mathrm{D}_{11 / 2}^{\circ} \\
a^{4} \mathrm{~F}_{21 / 2}-y^{4} \mathrm{~F}_{31 / 2}^{0}\end{array}$ & $\begin{array}{l}(0.00) 1.12 \\
(0.00) 0.93 \\
(0.00 \mathrm{~W}) 1.45 \mathrm{~A}^{2}\end{array}$ & $\begin{array}{l}(0.00) 1.12 \\
(0.00) 0.92 \\
(0.00) 1.55 \mathrm{~A}^{2}\end{array}$ \\
\hline $\begin{array}{r}5,598.52 \\
88.33 \\
70.37 \\
68.45 \\
65.70\end{array}$ & $\begin{array}{r}3 \\
100 \\
3 \\
30 \\
10\end{array}$ & $\begin{array}{l}20, \text { II } \\
5, \text { II A } \\
50, \text { II } \\
20, \text { II }\end{array}$ & $\begin{array}{l}856.92 \\
889.48 \\
947.16 \\
953.35 \\
962.22\end{array}$ & $\begin{array}{l}a^{4} \mathrm{~F}_{31 / 2}-y^{4} \mathrm{~F}_{41 / 2}^{0} \\
a^{2} \mathrm{D}_{11 / 2}-z^{4} \mathrm{G}_{21 / 2}^{1} \\
a^{4} \mathrm{~F}_{31 / 5}-z^{2} \mathrm{G}_{31 / 2}^{0} \\
a^{4} \mathrm{~F}_{21 / 2}-x^{2} \Psi_{21 / 2}^{0}\end{array}$ & $(0.44) 1.16$ & $(0.41) 1.15$ \\
\hline $\begin{array}{l}65.43 \\
62.54 \\
44.90 \\
41.25 \\
32.17\end{array}$ & $\begin{array}{r}10 \\
2 \\
3 \\
15 \\
4\end{array}$ & $\begin{array}{r}\text { 20, III } \\
2, \text { III } \\
6, \text { III } \\
20, \text { III } \\
3, \text { III }\end{array}$ & $\begin{array}{r}963.09 \\
17,972.42 \\
18,029.60 \\
041.47 \\
071.08\end{array}$ & $\begin{array}{l}a^{4} \mathrm{P}_{21 / 2}-y^{4} \mathrm{P}_{11 / 2}^{0} \\
a^{2} \mathrm{~F}_{21 / 2}-x^{4} \mathrm{~F}^{2} 11 / 2 \\
a^{2} \mathrm{P}_{11 / 2}-\mathrm{S}_{11 / 2}^{1} \\
0^{2} \mathrm{D}_{21 / 2}-w^{2} \mathrm{P}_{11 / 2}^{0} \\
a^{2} \mathrm{~F}_{21 / 2}-x^{4} \mathrm{D}_{31 / 2}^{0}\end{array}$ & $\begin{array}{l}(0.00) 1.52 \\
(0.28) 1.47 \\
(0.00) 1.25\end{array}$ & $\begin{array}{ll}(0.00) & 1.49 \\
(0.35) & 1.44 \\
(0.00) & 1.25\end{array}$ \\
\hline $\begin{array}{l}29.86 \\
26.51 \\
17.34 \\
15.28 \\
07.33\end{array}$ & $\begin{array}{r}2 \\
20 \\
5\end{array}$ & $\begin{array}{l}3, \text { III } \\
1, \text { V } \\
30, \text { III } \\
10, \text { III } \\
2, \text { III }\end{array}$ & $\begin{array}{l}078.63 \\
089.59 \\
119.66 \\
126.42 \\
152.59\end{array}$ & $\begin{array}{l}a^{2} \mathrm{G}_{31 / 2}-u^{2} \mathrm{~F}_{31 / 2}^{0} \\
z^{2} \mathrm{~F}_{21 / 2}^{2}-35 \\
a^{2} \mathrm{G}_{41 / 2}-u^{2} \mathrm{~F}_{31 / 2}^{0} \\
a^{4} \mathrm{P}_{11 / 2}-y^{4} \mathrm{P}_{01 / 2}^{01} \\
a^{4} \mathrm{P}_{11 / 2}-y^{4} \mathrm{P}_{11 / 2}^{\circ}\end{array}$ & $\begin{array}{l}(0.60) \\
(0.25) \\
(.4 .42\end{array}$ & $\begin{array}{l}(0.00) 1.10 \\
(0.30) 1.39,2.00\end{array}$ \\
\hline $\begin{array}{r}06.00 \\
03.80 \\
02.66 \\
02.24 \\
5,510.34\end{array}$ & $\begin{array}{r}20 \\
40 \\
5 \\
3 \\
200\end{array}$ & $\begin{array}{l}\text { 40, II } \\
80, \text { III } \\
\text { 10, III } \\
\text { 4, III } \\
300, \text { I }\end{array}$ & $\begin{array}{l}156.97 \\
164.23 \\
168.00 \\
169.38 \\
172.35\end{array}$ & $\begin{array}{l}a^{2} \mathrm{D}_{11 / 2}-z^{4} \mathrm{P}_{21 / 3}^{0} \\
a^{4} \mathrm{~F}_{41 / 2}-z^{2} \mathrm{G}_{41 / 2}^{0} \\
a^{4} \mathrm{~F}_{31 / 2}-x^{2} \mathrm{~F}_{31 / 2}^{0} \\
a^{2} \mathrm{G}_{41 / 2}-y^{4} \mathrm{G}_{41 / 2}^{0} \\
a^{2} \mathrm{D}_{11 / 2}-y^{2} \mathrm{D}_{11 / 2}^{0}\end{array}$ & $\begin{array}{l}(0.00 \mathrm{w}) 1.54 \mathrm{~h} \\
(0.70) 1.22 \mathrm{~B}\end{array}$ & $\begin{array}{l}(0.00) \\
(0.69) \\
1.23\end{array}$ \\
\hline $\begin{array}{r}5,498.70 \\
91.07 \\
75.17 \\
66.91 \\
55.14\end{array}$ & $\begin{array}{r}2 \\
5 \\
10 \\
2 \\
200\end{array}$ & $\begin{array}{l}2 \mathrm{n}, \text { III } \\
8, \text { III } \\
15, \text { III } \\
3, \text { III } \\
400, \text { I }\end{array}$ & $\begin{array}{l}181.08 \\
206.34 \\
259.21 \\
286.80 \\
326.25\end{array}$ & $\begin{array}{l}a^{2} \mathrm{P}_{1 / 2}-w^{2} \mathrm{P}_{11 / 2}^{0} \\
a^{2} \mathrm{~F}_{21 / 2}-x^{2} \mathrm{D}_{21 / 2}^{0} \\
a^{2} \mathrm{~F}_{31 / 2}-y^{4} \mathrm{P}_{21 / 2}^{0} \\
a^{2} \mathrm{D}_{21 / 2}-y^{2} \mathrm{D}_{11 / 2}^{0}\end{array}$ & $\begin{array}{l}(0.00) 1.08 \\
(0.00) 1.20\end{array}$ & $(0.02) 1.20$ \\
\hline $\begin{array}{r}37.55 \\
29.86 \\
22.10 \\
5,415.67 \\
5,390.63\end{array}$ & ? & $\begin{array}{l}\text { 5, III } \\
\text { 15, III } \\
\text { 3, III A } \\
\text { 8, III }\end{array}$ & $\begin{array}{l}385.54 \\
411.57 \\
437.93 \\
459.82 \\
545.56\end{array}$ & $\begin{array}{l}a^{4} \mathrm{P}_{1 / 2}-y^{4} \mathrm{P}_{01 / 2}^{0} \\
a^{4} \mathrm{P}_{1 / 2}^{1 / 2}-y^{4} \mathrm{P}_{11 / 2}^{0} \\
a^{4} \mathrm{~F}_{21 / 2}-z^{2} \mathrm{C}_{31 / 2}^{0} \\
a^{4} \mathrm{P}_{13 / 2}-x^{4} \mathrm{P}_{11 / 2}^{0} \\
a^{2} \mathrm{G}_{31 / 2}-w^{2} \mathrm{D}_{21 / 2}^{0}\end{array}$ & $\begin{array}{l}(0.00) 2.54 \\
(0.49) 1.04 \\
(0.36) 1.56 \mathrm{~W}\end{array}$ & $\begin{array}{l}(0.21) 2.50 \\
(0.55) 1.06,2.15 \\
(0.39) 1.56\end{array}$ \\
\hline
\end{tabular}


TABLE 6.-The arc spectrum of lanthanum ( $L a I)$-Continued

\begin{tabular}{|c|c|c|c|c|c|c|}
\hline \multirow{2}{*}{$\lambda_{\text {a ir I. A. }}$} & \multicolumn{2}{|c|}{$\begin{array}{l}\text { Intensities, tem- } \\
\text { perature class }\end{array}$} & \multirow{2}{*}{$\nu_{\nabla 80} \mathrm{~cm}^{-1}$} & \multirow{2}{*}{$\begin{array}{l}\text { Terin combi- } \\
\text { nation }\end{array}$} & \multicolumn{2}{|c|}{ Zeeman effects } \\
\hline & B. S. & K. \& C. & & & Observed & Computed \\
\hline $\begin{array}{l}80.00 \\
65.87 \\
59.70 \\
57.85 \\
30.64\end{array}$ & $\begin{array}{r}3 \\
4 \\
2 \\
25 \\
1\end{array}$ & $\begin{array}{l}5, \text { III } \\
8, \text { III } \\
2, \text { III A } \\
60, \text { III }\end{array}$ & $\begin{array}{l}582.21 \\
631.14 \\
652.59 \\
659.03 \\
754.27\end{array}$ & $\begin{array}{l}a^{2} \mathrm{G}_{31 / 2}-t^{2} \mathrm{~F}_{31 / 2}^{0} \\
a^{2} \mathrm{~F}_{235}-y^{4} \mathrm{P}_{11 / 2}^{1} \\
a^{4} \mathrm{~F}_{21 / 2}-x^{2} \mathrm{~F}_{31 / 2}^{0} \\
a^{4} \mathrm{P}_{21 / 2}-y^{4} \mathrm{P}_{21 / 2}^{0}\end{array}$ & $(0.00) 1.53$ & $(0.00) 1.54$ \\
\hline $\begin{array}{r}23.56 \\
21.34 \\
20.14 \\
07.52 \\
5,304.01\end{array}$ & $\begin{array}{r}3 \\
1 \\
3 \\
3 \\
20\end{array}$ & $\begin{array}{r}3, \text { III } \\
\text { 3, III } \\
3, \text { III } \\
30, \text { III }\end{array}$ & $\begin{array}{l}779.22 \\
787.04 \\
791.28 \\
835.96 \\
848.43\end{array}$ & $\begin{array}{l}b^{2} \mathrm{D}_{11 / 2}-w^{2} \mathrm{P}_{11 / 2}^{\circ} \\
a^{2} \mathrm{P}_{1 / 2}-w^{2} \mathrm{D}_{21 / 2}^{2} \\
a^{4} \mathrm{~F}_{31 / 2}-z^{2} \mathrm{G}_{41 / 2} \\
a^{4} \mathrm{P}_{11 / 2}-y^{4} \mathrm{P}_{21 / 2}^{\circ}\end{array}$ & & . \\
\hline $\begin{array}{r}5,287.45 \\
76.40 \\
71.18 \\
57.83 \\
53.45\end{array}$ & $\begin{array}{r}1 \\
5 \\
100 \\
4 \\
100\end{array}$ & $\begin{array}{l}1, \text { V } \\
10, \text { III } \\
150, \text { I } \\
3, \text { III } \\
100, \text { I }\end{array}$ & $\begin{array}{r}907.46 \\
947.06 \\
18,965.82 \\
19,013.98 \\
029.83\end{array}$ & $\begin{array}{l}b^{2} \mathrm{D}_{11 / 2}-w^{2} \mathrm{~F}_{21 / 2}^{0} \\
a^{2} \mathrm{D}_{21 / 2}-y^{2} \mathrm{P}_{11 / 2}^{2} \\
a^{2} \mathrm{D}_{21 / 2}-y^{4} \mathrm{~F}_{11 / 2}^{\circ}\end{array}$ & $\begin{array}{l}(0.00) 0.90 \\
(0.00) 1.39 \mathrm{~A}^{2} \\
(0.00) 1.07 \\
(0.23,0.69) 0.57,1.03, \\
1.49, \mathbf{1 . 9 5}\end{array}$ & $\begin{array}{l}(0.00) 0.90 . \\
(0.00) 1.44 \mathrm{~A}^{2} \\
(0.21,0.63) \quad 0.57,0.99 \\
1.41,1.83 .\end{array}$ \\
\hline $\begin{array}{r}40.81 \\
39.54 \\
34.27 \\
5,211.85 \\
5,190.34\end{array}$ & $\begin{array}{r}4 \\
4 \\
150 \\
150 \\
8\end{array}$ & $\begin{array}{l}\text { 2, III } \\
4, \text { III } \\
300, \text { II } \\
300, \text { II }\end{array}$ & $\begin{array}{l}075.72 \\
080.35 \\
099.56 \\
187.72 \\
261.21\end{array}$ & $\begin{array}{l}a^{2} \mathrm{~F}_{31 / 2}-y^{2} \mathrm{G}_{31 / 2}^{\circ} \\
a^{4} \mathrm{~F}_{41 / 2}-1_{31 / 2}^{\circ} \\
a^{4} \mathrm{~F}_{41 / 2}-y^{4} \mathrm{D}_{31 / 2}^{\circ}\end{array}$ & $\begin{array}{l}(0.00 \mathrm{~W}) 2.10 \mathrm{~A}^{2} \\
(0.00 \mathrm{~W}) 1.73 \mathrm{~A}^{2}\end{array}$ & $\begin{array}{l}(0.00) \\
(0.00) \\
1.71 \mathrm{~A}^{2}\end{array}$ \\
\hline $\begin{array}{l}83.91 \\
79.11 \\
77.30 \\
68.95 \\
67.79\end{array}$ & $\begin{array}{r}10 \\
2 \\
150 \\
2 \\
20\end{array}$ & $\begin{array}{l}20, \text { II } \\
2, \text { III } \\
300, \text { II } \\
20, \text { III }\end{array}$ & $\begin{array}{l}285.10 \\
302.98 \\
309.72 \\
340.92 \\
345.26\end{array}$ & $\begin{array}{l}a^{2} \mathrm{D}_{21 / 2}-y^{4} \mathrm{~F}_{21 / 2}^{0} \\
b^{2} \mathrm{D}_{11 / 2}-5_{1 / 2}^{\circ} \\
a^{4} \mathrm{~F}_{31 / 2}-y^{4} \mathrm{D}_{21 / 2}^{\circ} \\
a^{2} \mathrm{~F}_{31 / 2}-u^{2} \mathrm{~F}_{21 / 2}^{0} \\
a^{4} \mathrm{~F}_{41 / 2}-2_{11 / 2}^{\circ}\end{array}$ & $(0.00) 1.07$ & $(0.00) 1.06$ \\
\hline $\begin{array}{l}64.03 \\
61.54 \\
58.68\end{array}$ & $\begin{array}{r}2 \\
40\end{array}$ & $\begin{array}{l}1, \mathrm{~V} ? \\
80, \mathrm{I}\end{array}$ & $\begin{array}{l}359.34 \\
368.68 \\
379.42\end{array}$ & $\begin{array}{l}b^{2} \mathrm{D}_{21 / 2}-t^{2} \mathrm{~F}_{31 / 2}^{\circ} \\
a^{2} \mathrm{D}_{11 / 2}-y^{2} \mathrm{D}_{21 / 2}^{\circ}\end{array}$ & $\begin{array}{l}(0.19,0.57) 1.05,1.45 \text {, } \\
1.85\end{array}$ & $\begin{array}{l}(0.20,0.60) \\
1.38,1.78\end{array}-, 0.98$ \\
\hline $\begin{array}{l}52.31 \\
45.42\end{array}$ & $\begin{array}{r}1 \\
100\end{array}$ & 200, II & $\begin{array}{l}403.38 \\
429.36\end{array}$ & $\begin{array}{l}a^{2} \mathrm{~F}_{31 / 2}-y^{4} \mathrm{G}_{31 / 3}^{0} \\
a^{4} \mathrm{~F}_{21 / 2}-y^{4} \mathrm{D}_{1 / 2 / 2}^{0}\end{array}$ & $(0.00) 0.89$ & $\begin{array}{r}1.38,1.18 \\
(0.00) 0.90)\end{array}$ \\
\hline $\begin{array}{l}39.16 \\
35.42 \\
34.37 \\
29.81 \\
20.87\end{array}$ & $\begin{array}{r}3 \\
3 \\
2 \\
3 \\
10\end{array}$ & $\begin{array}{l}2, \mathrm{~V} \\
10, \mathrm{III}\end{array}$ & $\begin{array}{l}453.03 \\
467.20 \\
471.18 \\
488.48 \\
522.51\end{array}$ & $b^{2} \mathrm{D}_{11 / 2}-w^{2} \mathrm{D}_{11 / 2}$ & $(0.00) 0.92$ & $(0.03) 0.90$ \\
\hline $\begin{array}{r}09.12 \\
06.23 \\
5,103.11 \\
5,096.59 \\
86.22\end{array}$ & $\begin{array}{r}3 \\
100 \\
3 \\
2 \\
2\end{array}$ & $\begin{array}{l}2, \text { III A } \\
150, \text { II } \\
2, \text { III } \\
\text { 1, IV? }\end{array}$ & $\begin{array}{l}567.40 \\
578.48 \\
590.45 \\
615.51 \\
655.50\end{array}$ & $\begin{array}{l}a^{2} \mathrm{~F}_{31 / 2}-y^{2} \mathrm{G}_{41 / 2}^{\circ} \\
a^{4} \mathrm{~F}_{11 / 2}-y^{4} \mathrm{D}_{01 / 2}^{\circ} \\
z^{4} \mathrm{~F}_{21 / 2}^{\circ}-35\end{array}$ & $(0.19) 0.62 \mathrm{~A}^{2} ?$ & $(0.19) 0.22,0.60$ \\
\hline $\begin{array}{l}79.37 \\
78.92 \\
72.10 \\
67.90 \\
56.46\end{array}$ & $\begin{array}{r}4 \\
3 \\
3 \\
15 \\
60\end{array}$ & $\begin{array}{l}5, \text { IV } \\
2, \text { V? } \\
\text { 1, III A } \\
\text { 15, III } \\
80, \text { II }\end{array}$ & $\begin{array}{l}682.01 \\
683.76 \\
710.22 \\
720.56 \\
771.18\end{array}$ & $\begin{array}{l}a^{2} \mathrm{D}_{21 / 2}-y^{4} \mathrm{~F}_{31 / 2}^{\circ} \\
a^{4} \mathrm{~F}_{31 / 2}-1_{31 / 2}^{1} \\
a^{4} \mathrm{~F}_{11 / 2}-y^{1} \mathrm{D}_{11 / 2}^{\circ}\end{array}$ & $(1.20) 0.00,0.79,1.57$ & $(0.39,1.19) 0.02,0.81$, \\
\hline $\begin{array}{l}52.10 \\
50.57\end{array}$ & $\begin{array}{r}1 \\
60\end{array}$ & 80, II & $\begin{array}{l}788.25 \\
794.24\end{array}$ & $\begin{array}{l}b^{2} \mathrm{D}_{21 / 2}-v^{2} \mathrm{D}_{11 / 2}^{i} \\
a^{4} \mathrm{~F}_{21 / 2}-y^{4} \mathrm{D}_{21 / 2}^{0}\end{array}$ & $(0.80) 1.18 \mathrm{~B}$ & $(-,-, 0.79)-, 0.88$ \\
\hline $\begin{array}{l}46.87 \\
37.60 \\
33.24\end{array}$ & $\begin{array}{r}30 \\
2 \\
2\end{array}$ & 60, III & $\begin{array}{l}808.75 \\
845.20 \\
862.40\end{array}$ & $\begin{array}{l}a^{4} \mathrm{~F}_{31 / 2}-y^{4} \mathrm{D}_{31 / 2}^{0} \\
a^{2} \mathrm{P}_{11 / 2}-6_{01 / 2}^{0}\end{array}$ & $(0.00 \mathrm{w}) 1.24$ & $(0.00) 1.22$ \\
\hline $\begin{array}{r}19.50 \\
5,001.78 \\
4,995.95 \\
94.64 \\
93.87\end{array}$ & $\begin{array}{r}10 \\
20 \\
1 \\
2 \\
15\end{array}$ & $\begin{array}{l}8, \text { III } \\
10, \text { III A } \\
1, \text { III A } \\
20, \text { II }\end{array}$ & $\begin{array}{r}916.76 \\
19,987.32 \\
20,010.64 \\
015.90 \\
018.98\end{array}$ & $\begin{array}{l}a^{2} \mathrm{~F}_{31 / 2}-w^{2} \mathrm{~F}_{31 / 2}^{\circ} \\
a^{2} \mathrm{~F}_{21 / 2}-y^{4} \mathrm{G}_{21 / 2}^{2} \\
b^{2} \mathrm{D}_{21 / 2}-w^{4} \mathrm{D}_{11 / 2}^{1} \\
a^{2} \mathrm{D}_{11 / 2}-y^{2} \mathrm{P}_{11 / 2}^{1}\end{array}$ & $\frac{1}{1}$ & \\
\hline $\begin{array}{l}84.92 \\
84.63 \\
83.56 \\
77.95\end{array}$ & $\begin{array}{l}3 \\
1 \\
2 \\
8\end{array}$ & $2, \mathrm{IV} ?$ & $\begin{array}{l}054.92 \\
056.09 \\
060.40 \\
083.01\end{array}$ & $\begin{array}{l}a^{1} \mathrm{~F}_{31 / 2}-3_{21 / 2}^{\circ} \\
a^{2} \mathrm{P}_{1 / 2}-v^{2} \mathrm{D}_{21 / 2}^{2} \\
b^{2} \mathrm{D}_{1 / 2}-w^{2} \mathrm{D}_{21 / 2}^{1} \\
a^{2} \mathrm{D}_{11 / 2}-y^{4} \mathrm{~F}_{11 / 2}^{\circ}\end{array}$ & & \\
\hline 68.59 & $4 \mathrm{~d}$ & $2\left\{\begin{array}{l}\mathrm{III} \\
\mathrm{V}\end{array}\right\}$ & 120.84 & $a^{2} \mathrm{~F}_{21 / 2}-y^{2} \mathrm{G}_{3}^{0}$ & & \\
\hline
\end{tabular}


TABLE 6.-The arc spectrum of lanthanum ( $L a I)$-Continued

\begin{tabular}{|c|c|c|c|c|c|c|}
\hline \multirow{2}{*}{$\lambda_{\text {air I. A. }}$} & \multicolumn{2}{|c|}{$\begin{array}{l}\text { Intensities, tem- } \\
\text { perature class }\end{array}$} & \multirow{2}{*}{$\nu_{\nabla_{\mathrm{aO}}} \mathrm{cm}^{-1}$} & \multirow{2}{*}{$\begin{array}{l}\text { Term combi- } \\
\text { nation }\end{array}$} & \multicolumn{2}{|c|}{ Zeeman effects } \\
\hline & B. S. & K. \& C. & & & Observed & Computed \\
\hline $\begin{array}{l}64.84 \\
57.77 \\
49.76 \\
45.84 \\
25.40\end{array}$ & $\begin{array}{r}3 \\
4 \\
50 \\
3 \\
3\end{array}$ & $\begin{array}{l}4, \text { III A } \\
200, \text { I } \\
5, \text { III A } \\
2, \text { IV }\end{array}$ & $\begin{array}{l}136.03 \\
164.75 \\
197.38 \\
213.39 \\
297.27\end{array}$ & 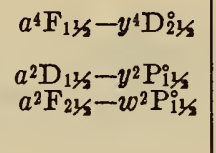 & $(0.00) 0.84$ & $(0.00) 0.84$ \\
\hline $\begin{array}{r}16.62 \\
05.13 \\
4,901.87 \\
4,894.24 \\
87.60\end{array}$ & $\begin{array}{r}3 \\
4 \\
15 \\
2 \\
4\end{array}$ & $\begin{array}{l}\text { 1, III? A } \\
4, \text { III A } \\
25, \text { I } \\
1, \text { III } \\
5, \text { III }\end{array}$ & $\begin{array}{l}333.52 \\
381.15 \\
394.70 \\
426.49 \\
454.24\end{array}$ & $\begin{array}{l}a^{2} \mathrm{~F}_{21 / 5}-u^{2} \mathrm{~F}_{21 / 5}^{\circ} \\
a^{2} \mathrm{D}_{21 / 5}-z^{2} \mathrm{G}_{31 / 5}^{\circ} \\
a^{2} \mathrm{~F}_{31 / 5}-w^{2} \mathrm{D}_{21 / 5}^{\circ}\end{array}$ & $(0.00) 0.94$ & $(0.00) 0.92$ \\
\hline $\begin{array}{l}86.82 \\
81.94 \\
78.86 \\
70.56 \\
68.90\end{array}$ & $\begin{array}{r}3 \\
1 \\
10 \\
5 \\
3\end{array}$ & $\begin{array}{c}2, \text { IV } \\
15, \text { III } \\
\text { 5, III } \\
2, \text { IV? }\end{array}$ & $\begin{array}{l}457.51 \\
477.96 \\
490.89 \\
525.81 \\
532.80\end{array}$ & $\begin{array}{l}a^{4} \mathrm{P}_{11 / 3}-w^{2} \mathrm{D}_{13 / 3}^{\circ} \\
a^{2} \mathrm{~F}_{31 / 3}-t^{2} \mathrm{~F}_{31 / 3}^{\circ} \\
b^{2} \mathrm{D}_{11 / 3}-v^{2} \mathrm{D}_{11 / 3}^{10}\end{array}$ & $(0.00) 1.10 \mathrm{R}$ & $(0.18) 1.10$ \\
\hline $\begin{array}{l}67.37 \\
54.95 \\
50.81 \\
39.51 \\
17.55\end{array}$ & $\begin{array}{r}3 \\
8 \\
20 \\
20 \\
3\end{array}$ & $\begin{array}{l}\text { 8, III } \\
20, \mathrm{I} \\
25, \mathrm{II}\end{array}$ & $\begin{array}{l}539.26 \\
591.80 \\
609.37 \\
657.49 \\
751.66\end{array}$ & $\begin{array}{l}a^{4} \mathrm{~F}_{21 / 3}-3_{21 / 3}^{\circ} \\
b^{2} \mathrm{D}_{21 / 3}-v^{2} \mathrm{D}_{23 / 2}^{\circ} \\
a^{2} \mathrm{D}_{21 / 2}-x^{2} \mathrm{~F}_{33 / 2}^{0} \\
a^{2} \mathrm{~F}_{21 / 2}-t^{2} \mathrm{~F}_{23 / 3}^{3}\end{array}$ & $\begin{array}{l}(0.00) 1.21 \mathrm{R} \\
(0.00) 0.89\end{array}$ & $\begin{array}{l}(0.35) 1.21 \\
(0.04) 0.89\end{array}$ \\
\hline $\begin{array}{r}17.17 \\
4,800.24 \\
4,799.99 \\
92.46 \\
91.77\end{array}$ & $\begin{array}{r}10 \\
9 \\
8 \\
1 \\
1\end{array}$ & $\begin{array}{l}\text { 4, IV? } \\
8, \text { III } \\
8, \text { III } \\
\text { tr, III A }\end{array}$ & $\begin{array}{l}753.29 \\
826.49 \\
827.57 \\
860.30 \\
863.30\end{array}$ & 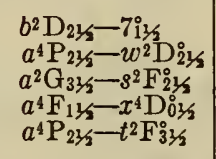 & & \\
\hline $\begin{array}{l}91.39 \\
79.89 \\
75.14 \\
70.43 \\
67.80\end{array}$ & $\begin{array}{c}5 \\
4 \\
3 \\
10 \\
1 \mathrm{~h}\end{array}$ & $\begin{array}{r}5, \text { II } \\
4, \text { II } \\
2, \mathrm{~V} \\
15, \text { II }\end{array}$ & $\begin{array}{l}864.96 \\
915.16 \\
935.96 \\
956.63 \\
968.19\end{array}$ & 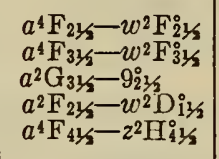 & $(0.00) 0.93 \mathrm{R}$ & $(0.00) 0.89$ \\
\hline $\begin{array}{l}66.89 \\
59.71 \\
57.14 \\
56.97 \\
53.11\end{array}$ & $\begin{array}{r}60 \\
2 \\
3 \\
2\end{array}$ & $\begin{array}{l}100, \mathrm{I} \\
2, \mathrm{IV} ? \\
2, \mathrm{IV} \\
1, \mathrm{~V} \\
1, \mathrm{IV}\end{array}$ & $\begin{array}{r}20,972.19 \\
21,003.83 \\
015.18 \\
015.93 \\
032.99\end{array}$ & $\begin{array}{l}a^{2} \mathrm{D}_{11 / 3}-x^{2} \mathrm{~F}_{21 / 3}^{\circ} \\
a^{2} \mathrm{G}_{31 / 2}-s^{2} \mathrm{~F}_{31 / 2}^{0} \\
a^{4} \mathrm{P}_{13 / 2}-w^{2} \mathrm{D}_{21 / 2}^{\circ}\end{array}$ & $(0.00) 0.99$ & $(0.00) 0.98$ \\
\hline $\begin{array}{l}52.41 \\
50.41 \\
33.82 \\
29.09 \\
23.72\end{array}$ & $\begin{array}{r}3 \\
10 \\
8 \\
1 \\
2\end{array}$ & $\begin{array}{l}\text { 3, III } \\
15, \text { III } \\
4, \text { V? } \\
\text { 1, V } \\
3, \text { II }\end{array}$ & $\begin{array}{l}036.09 \\
044.95 \\
118.70 \\
139.82 \\
163.85\end{array}$ & $\begin{array}{l}a^{4} \mathrm{~F}_{21 / 5}-x^{4} \mathrm{D}_{21 / 2}^{\circ} \\
a^{2} \mathrm{G}_{43 / 3}-s^{2} \mathrm{~F}_{31 / 3}^{\circ} \\
b^{2} \mathrm{D}_{13 / 5}-6_{01 / 2}^{0} \\
a^{4} \mathrm{~F}_{23 / 2}-4_{11 / 2}^{\circ}\end{array}$ & $(0.00) 1.04 \mathrm{R}$ & $(0.00) 1.02$ \\
\hline $\begin{array}{r}14.14 \\
08.18 \\
02.64 \\
4,700.26 \\
4,695.30\end{array}$ & $\begin{array}{l}4 \\
8 \\
8 \\
8 \\
3\end{array}$ & $\begin{array}{l}\text { 5, I } \\
\text { 8, III? } \\
10, \text { I } \\
\text { 8, III? }\end{array}$ & $\begin{array}{l}206.86 \\
233.71 \\
258.72 \\
269.48 \\
291.95\end{array}$ & $\begin{array}{l}a^{4} \mathrm{~F}_{11 / 2}-w^{2} \mathrm{~F}_{21 / 2}^{\circ} \\
b^{2} \mathrm{D}_{21 / 2}-8_{11 / 2}^{1} \\
a^{4} \mathrm{~F}_{41 / 5}-x^{4} \mathrm{~F}_{31 / 2}^{\circ} \\
a^{4} \mathrm{P}_{21 / 5}-v^{2} \mathrm{D}_{1 / 2}\end{array}$ & $(0.00) 0.97 \mathrm{R}$ & $(0.00) 1.15$ \\
\hline $\begin{array}{l}60.70 \\
53.90 \\
52.07 \\
50.32 \\
48.64\end{array}$ & $\begin{array}{r}8 \\
4 \\
15 \\
12 \\
30\end{array}$ & $\begin{array}{l}8, \text { III } \\
20, \text { I } \\
15, \text { I } \\
40, \text { I }\end{array}$ & $\begin{array}{l}450.02 \\
481.36 \\
489.81 \\
497.90 \\
505.67\end{array}$ & $\begin{array}{l}a^{2} \mathrm{~F}_{31 / 5}-w^{4} \mathrm{D}_{21 / 5}^{\circ} \\
a^{4} \mathrm{P}_{11 / 5}-v^{2} \mathrm{D}_{11 / 5}^{1} \\
a^{4} \mathrm{~F}_{31 / 5}-x^{4} \mathrm{~F}_{21 / 5}^{\circ} \\
a^{4} \mathrm{~F}_{23 / 5}-v^{2} \mathrm{~F}_{21 / 5}^{2} \\
a^{4} \mathrm{~F}_{11 / 5}-4_{11 / 5}^{1}\end{array}$ & $\begin{array}{l}(0.00) 0.92 \mathrm{R} \\
(0.30) 1.13 \\
(0.48)\end{array}$ & $\begin{array}{l}(0.00) 0.92 \\
(0.37) 1.12 \\
(0.15,0.46) 0.26,0.57, \\
0.87\end{array}$ \\
\hline $\begin{array}{l}46.33 \\
43.11 \\
27.35 \\
15.06 \\
05.08\end{array}$ & $\begin{array}{r}10 \\
5 \\
2 \\
8 \\
6\end{array}$ & $\begin{array}{l}\text { 12, III } \\
5, \text { III } \\
15, \text { III } \\
10, \text { III }\end{array}$ & $\begin{array}{l}516.36 \\
531.28 \\
604.61 \\
662.14 \\
709.09\end{array}$ & 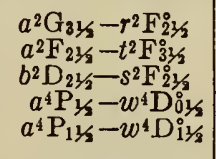 & $\begin{array}{l}(0.00) 0.89 \mathrm{R} \\
(1.31) 1.37 \mathrm{R}\end{array}$ & $\begin{array}{l}(0.00) 0.95 \\
(1.35) 1.35\end{array}$ \\
\hline $\begin{array}{r}04.24 \\
4,602.04 \\
4,596.19 \\
89.89 \\
81.20\end{array}$ & $\begin{array}{r}6 \\
10 \\
6 \\
5 \\
10\end{array}$ & $\begin{array}{l}\text { 10, III } \\
20, \text { III } \\
10, \text { I } \\
12, \text { III }\end{array}$ & $\begin{array}{l}713.05 \\
723.43 \\
751.08 \\
780.93 \\
822.25\end{array}$ & 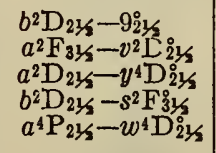 & & \\
\hline $\begin{array}{l}70.02 \\
67.90 \\
64.85 \\
52.47 \\
50.76\end{array}$ & $\begin{array}{r}60 \\
50 \\
6 \\
8 \\
8\end{array}$ & $\begin{array}{l}250, \text { I } \\
200, \text { I } \\
12, \text { III } \\
8, \text { II A } \\
10, \text { III A }\end{array}$ & $\begin{array}{l}875.63 \\
885.78 \\
900.41 \\
959.96 \\
968.21\end{array}$ & 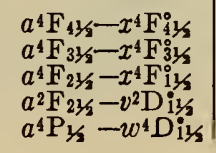 & $\begin{array}{l}(0.00) 1.34 \\
(0.00) 1.24 \\
(0.00) 1.42 \mathrm{R} \\
(0.00) 0.87 \\
(0.78) 0.38 \mathrm{R}\end{array}$ & $\begin{array}{l}(0.11) 1.34 \\
(0.02) 1.23 \\
(0.15,045) 1.18,1.48 \\
(0.00) 0.87 \\
(0.77) 0.38,1.93\end{array}$ \\
\hline
\end{tabular}


TABLE 6.-The arc spectrum of lanthanum ( $L a I)$-Continued

\begin{tabular}{|c|c|c|c|c|c|c|}
\hline \multirow{2}{*}{$\lambda_{\text {air I. A. }}$. } & \multicolumn{2}{|c|}{$\begin{array}{l}\text { Intensities, tem- } \\
\text { perature class }\end{array}$} & \multirow{2}{*}{$\nu \nabla 80 \mathrm{~cm}^{-1}$} & \multirow{2}{*}{$\begin{array}{l}\text { Term combi- } \\
\text { nation }\end{array}$} & \multicolumn{2}{|c|}{ Zeeman effects } \\
\hline & B. $\mathrm{s}$. & K. \& C. & & & Observed & Computed \\
\hline $\begin{array}{l}50.16 \\
49.50 \\
41.78 \\
37.57 \\
28.88\end{array}$ & $\begin{array}{r}4 \\
40 \\
10 \\
2 \\
3\end{array}$ & $\begin{array}{l}5, \text { III A } \\
50, \text { I } \\
15, \text { III }\end{array}$ & $\begin{array}{r}971.11 \\
21,974.30 \\
22,011.65 \\
032.07 \\
074.34\end{array}$ & $\begin{array}{l}a^{4} \mathrm{~F}_{11 / 2}-z^{4} \mathrm{~S}_{11 / 2} \\
a^{4} \mathrm{~F}_{21 / 2}-x^{4} \mathrm{~F}_{21 / 2}^{\circ} \\
a^{4} \mathrm{P}_{11 / 2}-w^{4} \mathrm{D}_{21 / 2}^{21} \\
a^{2} \mathrm{P}_{11 / 2}-u^{2} \mathrm{D}_{13 / 2}^{\mathrm{o}} \\
a^{4} \mathrm{P}_{11 / 2}-6_{01 / 2}^{0}\end{array}$ & $(0.00) 1.08$ & $(0.17) 1.07$ \\
\hline $\begin{array}{c}07.4 \\
01.57 \\
4,500.21\end{array}$ & $\begin{array}{r}2 \\
6 \\
30\end{array}$ & $\begin{array}{l}10, \text { II A } \\
40, \text { II }\end{array}$ & $\begin{array}{l}179.5 \\
208.26 \\
214.97\end{array}$ & $\begin{array}{l}a^{2} \mathrm{G}_{31 / 2}-r^{2} \mathrm{~F}_{31 / 2}^{0} \\
a^{4} \mathrm{~F}_{21 / 2}-x^{2} \mathrm{D}_{21 / 2}^{\circ} \\
a^{4} \mathrm{P}_{21 / 2}-w^{4} \mathrm{D}_{31 / 2}^{10}\end{array}$ & $(0.00 \mathrm{w}) 1.10 \mathrm{~A}^{1}$ & $(0.12,0.37-) 0.67$, \\
\hline $\begin{array}{r}4,499.04 \\
94.71\end{array}$ & $\begin{array}{l}10 \\
20\end{array}$ & $\begin{array}{l}10, \text { III } \\
30, I\end{array}$ & $\begin{array}{l}220.75 \\
242.16\end{array}$ & $\begin{array}{l}a^{2} \mathrm{G}_{413 / 2}-r^{2} \mathrm{~F}_{31 / 2}^{\circ} \\
a^{4} \mathrm{~F}_{1 / 2}-x^{4} \mathrm{~F}_{1 / 2}^{11 / 2}\end{array}$ & $\begin{array}{ll}(0.00) & 1.02 \\
(0.51) & 0.27,0.58,0.91 \\
\text { us } & \end{array}$ & $\begin{array}{l}(0.00) 1.02 \\
(0.16,0.47) \\
0.88\end{array}$ \\
\hline $\begin{array}{l}93.81 \\
93.11 \\
91.76 \\
86.06 \\
79.82\end{array}$ & $\begin{array}{r}5 \\
15 \\
10 \\
10 \\
6\end{array}$ & $\begin{array}{l}10, \text { I A } \\
25, \text { I } \\
15, \text { III } \\
20, \text { III } \\
15, \text { II A }\end{array}$ & $\begin{array}{l}246.61 \\
250.08 \\
256.76 \\
285.04 \\
316.08\end{array}$ & $\begin{array}{l}a^{2} \mathrm{D}_{11 / 2}-y^{4} \mathrm{D}_{031 / 2}^{\circ} \\
a^{2} \mathrm{D}_{21 / 2}-y^{4} \mathrm{D}_{31 / 2}^{\circ} \\
a^{4} \mathrm{P}_{21 / 2}-7_{1}^{1} 1 / 2 \\
a^{4} \mathrm{P}_{11 / 2}-v^{2} \mathrm{D}_{21 / 2}^{2} \\
a^{4} \mathrm{~F}_{13 / 2}-x^{4} \mathrm{~F}_{21 / 2}^{\circ}\end{array}$ & $\begin{array}{l}(0.00) 0.72 \mathrm{R} \\
(0.00) 1.16 \\
(0.00) 1.52\end{array}$ & $\begin{array}{l}(0.38) 0.42,1.17 \\
(0.04) 1.21 \\
(0.00) 1.54\end{array}$ \\
\hline $\begin{array}{l}74.54 \\
68.97 \\
55.21 \\
53.85 \\
52.15\end{array}$ & $\begin{array}{r}4 \\
10 \\
3 \\
15\end{array}$ & $\begin{array}{l}5, \text { III A } \\
25, \text { II } \\
10, \text { II A } \\
2, \text { IV A } \\
30, \text { II }\end{array}$ & $\begin{array}{l}342.42 \\
370.26 \\
439.35 \\
446.20 \\
449.78\end{array}$ & $\begin{array}{l}b^{2} \mathrm{D}_{13 / 2}-s^{2} \mathrm{~F}_{21 / 2}^{\circ} \\
a^{4} \mathrm{~F}_{21 / 2}-x^{4} \mathrm{~F}_{31 / 2}^{\circ} \\
a^{2} \mathrm{D}_{11 / 2}-y^{4} \mathrm{D}_{11 / 2}^{\circ} \\
a^{4} \mathrm{P}_{11 / 2}-7_{11 / 2}^{\circ} \\
a^{2} \mathrm{G}_{31 / 2}-y^{2} \mathrm{H}_{41 / 2}^{\circ}\end{array}$ & $(0.00) 0.98$ & $(0.00) 0.97$ \\
\hline $\begin{array}{l}45.12 \\
43.94 \\
42.68 \\
23.90 \\
17.14\end{array}$ & $\begin{array}{r}2 \\
5 \\
6 \\
20 \\
2\end{array}$ & $\begin{array}{l}\text { 2, III A } \\
10, \text { I } \\
12, \text { II } \\
30, \text { II } \\
6 \text { n, III }\end{array}$ & $\begin{array}{l}490.29 \\
496.26 \\
502.64 \\
598.16 \\
632.75\end{array}$ & $\begin{array}{c}a^{2} \mathrm{~F}_{21 / 2}-w^{4} \mathrm{D}_{21 / 2}^{\circ} \\
a^{2} \mathrm{D}_{23 / 2}-3_{21 / 2}^{\circ} \\
a^{4} \mathrm{~F}_{31 / 2}-x^{4} \mathrm{~F}_{41 / 2}^{\circ} \\
a^{2} \mathrm{G}_{41 / 2}-y^{2} \mathrm{H}_{51 / 2}^{\circ} \\
a^{4} \mathrm{~F}_{21 / 2}-y^{4} \mathrm{P}_{1 / 3}^{0}\end{array}$ & $(0.00) 1.09$ & $(0.00) 1.09$ \\
\hline $\begin{array}{r}13.45 \\
03.02 \\
4,402.64 \\
4,397.04 \\
96.79\end{array}$ & $\begin{array}{l}2 \\
5\end{array}$ & $\begin{array}{l}\text { 2, III A } \\
\text { 8, III A } \\
15 \text {, III } \\
2 \text {, IV A } \\
4 \text {, IV A }\end{array}$ & $\begin{array}{l}651.67 \\
705.33 \\
707.29 \\
736.21 \\
737.50\end{array}$ & $\begin{array}{c}a^{2} \mathrm{D}_{21 / 2}-x^{4} \mathrm{D}_{11 / 2}^{0} \\
a^{4} \mathrm{P}_{1 / 2}-7_{11 / 2}^{1} \\
a^{2} \mathrm{P}_{1 / 2}-u^{2} \mathrm{D}_{11 / 2}^{1} \\
a^{2} \mathrm{~F}_{31 / 2}-s^{2} \mathrm{~F}_{21 / 2}^{01} \\
a^{4} \mathrm{P}_{21 / 2}-8_{1 / 2}^{0}\end{array}$ & $(0.00) 0.86$ & $(0.00) 0.86$ \\
\hline $\begin{array}{l}96.31 \\
93.52 \\
89.87 \\
80.55 \\
60.86\end{array}$ & $\begin{array}{l}2 \\
6 \\
4 \\
2\end{array}$ & $\begin{array}{l}2, \text { IV } \\
4, \text { III } \\
15, \text { III } \\
12, \text { II A } \\
2, \text { III A }\end{array}$ & $\begin{array}{l}739.98 \\
754.42 \\
773.34 \\
821.79 \\
924.84\end{array}$ & $\begin{array}{l}a^{2} \mathrm{P}_{11 / 2}-u^{2} \mathrm{D}_{21 / 2}^{\circ} \\
a^{2} \mathrm{D}_{21 / 2}-w^{2} \mathrm{~F}_{21 / 2}^{\circ} \\
a^{2} \mathrm{~F}_{21 / 3}-7_{1}^{\circ} 1 / 2\end{array}$ & $(0.00) 1.07$ & $(0.00) 1.06$ \\
\hline $\begin{array}{l}60.49 \\
57.88 \\
54.79 \\
40.72 \\
39.93\end{array}$ & $\begin{array}{r}2 \\
2 \\
20 \\
10 \\
5\end{array}$ & $\begin{array}{l}2, \text { III A } \\
2, \text { III A } \\
25, \text { III } \\
15, \text { III } \\
6 \text {, III A }\end{array}$ & $\begin{array}{r}926.78 \\
340.51 \\
22,956.79 \\
23,031.20 \\
035.39\end{array}$ & $\begin{array}{l}a^{4} \mathrm{P}_{11 / 2}-8_{11 / 2}^{\circ} \\
a^{4} \mathrm{~F}_{21 / 2}-x^{2} \mathrm{P}_{11 / 2} \\
b^{2} \mathrm{D}_{11 / 2}-r^{2} \mathrm{~F}_{21 / 2}^{\circ} \\
b^{2} \mathrm{D}_{11 / 2}-r^{2} \mathrm{~F}_{21 / 2}^{\circ}\end{array}$ & $(0.00) 0.90$ & $(0.00) 0.87$ \\
\hline $\begin{array}{r}26.19 \\
11.73 \\
06.00 \\
4,300.62 \\
4,291.00\end{array}$ & $\begin{array}{c}2 \\
5 \\
6 \\
\mathrm{HNR} \\
2\end{array}$ & $\begin{array}{l}4, \text { III A } \\
6, \text { II A } \\
3\end{array}$ & $\begin{array}{l}108.55 \\
186.05 \\
216.90 \\
245.94 \\
298.06\end{array}$ & $\begin{array}{c}a^{4} \mathrm{P}_{21 / 2}-s^{2} \mathrm{~F}_{21 / 2}^{\circ} \\
a^{4} \mathrm{P}_{1 / 2}-8^{1} 1 / 2 \\
a^{4} \mathrm{P}_{21 / 2}-9^{2} 1 / 2 \\
a^{2} \mathrm{P}_{1 / 2}=v^{2} \mathrm{P}_{01 / 2}^{\circ} \\
a^{4} \mathrm{P}_{11 / 2}-s^{2} \mathrm{~F}_{21 / 2}^{2}\end{array}$ & $\begin{array}{l}(0.59) \\
0.96 \mathrm{R} \\
(0.14) 1.49\end{array}$ & $\begin{array}{l}(0.58) 0.96,2.12 \\
(0.20) 1.50\end{array}$ \\
\hline $\begin{array}{l}89.65 \\
89.01 \\
80.27 \\
71.14 \\
67.74\end{array}$ & $\begin{array}{c}\text { HNR } \\
\text { HNR } \\
60 \\
4 \\
2\end{array}$ & $\stackrel{1}{2}$ & $\begin{array}{l}305.39 \\
308.87 \\
356.46 \\
406.39 \\
425.04\end{array}$ & $\begin{array}{l}b^{2} \mathrm{D}_{11 / 2}-u^{2} \mathrm{D}_{11 / 2}^{\circ} \\
b^{2} \mathrm{D}_{21 / 2}-u^{2} \mathrm{D}_{21 / 2}^{\circ} \\
a^{2} \mathrm{D}_{21 / 2}-w^{2} \mathrm{~F}_{31 / 2}^{\circ} \\
a^{4} \mathrm{P}_{11 / 2}-9_{21}^{\circ} / / 2 \\
a^{2} \mathrm{~F}_{33 / 2}-r^{2} \mathrm{~F}_{21 / 2}^{\circ}\end{array}$ & $(0.00) 1.14$ & $(0.00) 1.13$ \\
\hline $\begin{array}{r}62.35 \\
56.92 \\
38.59 \\
4,216.54 \\
4,192.72\end{array}$ & $\begin{array}{r}10 \\
6 \\
4 \\
4 \\
\text { HNR }\end{array}$ & $\begin{array}{l}\text { 15, II A } \\
6, \text { III A } \\
10, \text { III A } \\
2\end{array}$ & $\begin{array}{l}454.66 \\
484.58 \\
586.13 \\
709.47 \\
844.17\end{array}$ & $\begin{array}{l}a^{2} \mathrm{D}_{21 / 2}-v^{2} \mathrm{~F}_{21 / 2}^{0} \\
a^{2} \mathrm{P}_{11 / 2}-v^{2} \mathrm{P}_{11 / 2}^{0} \\
a^{2} \mathrm{D}_{21 / 2}-z^{4} \mathrm{~S}_{11 / 2}^{0} \\
a^{2} \mathrm{D}_{21 / 2}-x^{2} \mathrm{D}_{11 / 2}^{\circ} \\
b^{2} \mathrm{D}_{11 / 2}-v^{2} \mathrm{P}_{01 / 2}^{\circ}\end{array}$ & $\begin{array}{l}(0.00 \mathrm{w}) 1.19 \\
(0.00) 1.29 \mathrm{R}\end{array}$ & $\begin{array}{l}(0.00) 1.20 \\
(0.08) 1.29\end{array}$ \\
\hline $\begin{array}{l}87.31 \\
77.48 \\
72.32 \\
71.13 \\
63.31\end{array}$ & $\begin{array}{r}50 \\
15 \\
6 \\
5 \\
5\end{array}$ & $\begin{array}{l}\text { 125, I } \\
30, \text { I } \\
\text { 10, III A } \\
\text { 8, III A } \\
\text { 8, III A }\end{array}$ & $\begin{array}{r}874.98 \\
931.15 \\
9 € 9.75 \\
23,907.58 \\
24.012 .60\end{array}$ & $\begin{array}{l}a^{2} \mathrm{D}_{11 / 2}-w^{2} \mathrm{~F}_{21 / 2}^{\circ} \\
a^{2} \mathrm{~N}_{21 / 2}-x^{4} \mathrm{~F}_{21 / 2}^{\circ} \\
a^{4} \mathrm{~F}_{31 / 2}-y^{4} \mathrm{G}_{31 / 2}^{\circ} \\
a^{4} \mathrm{~F}_{41 / 2}-y^{4} \mathrm{G}_{41 / 2}^{0} \\
a^{4} \mathrm{~F}_{21 / 2}-y^{4} \mathrm{G}_{21 / 2}^{0}\end{array}$ & $\begin{array}{l}\left(0.00 \text { w) } 1.16 \mathrm{~A}^{2}\right. \\
(0.00) 1.50(?)\end{array}$ & $\begin{array}{l}(0.00) 1.16 \mathrm{~A}^{2} \\
(0.20) 1.16\end{array}$ \\
\hline $\begin{array}{l}60.26 \\
57.52\end{array}$ & $\begin{array}{r}20 \\
6\end{array}$ & $\begin{array}{l}30, \text { I } \\
10, \text { II A }\end{array}$ & $\begin{array}{l}030.21 \\
046.04\end{array}$ & $\begin{array}{l}a^{2} \mathrm{D}_{21 / 2}-x^{4} \mathrm{D}_{31 / 2}^{0} \\
a^{2} \mathrm{D}_{135}-x^{4} \mathrm{D}_{13 / 2}^{\circ}\end{array}$ & $(0.00 \mathrm{w}) 1.65 \mathrm{w}$ & $(0.00) 1.67$ \\
\hline
\end{tabular}


TABLE 6.-The arc spectrum of lanthanum (La I)-Continued

\begin{tabular}{|c|c|c|c|c|c|c|}
\hline \multirow{2}{*}{$\lambda_{\text {sir }}$ I. A. } & \multicolumn{2}{|c|}{$\begin{array}{l}\text { Intensities, tem- } \\
\text { perature class }\end{array}$} & \multirow{2}{*}{$\nu_{\nabla \mathrm{ac}} \mathrm{cm}^{-1}$} & \multirow{2}{*}{$\begin{array}{l}\text { Terin combi- } \\
\text { nation }\end{array}$} & \multicolumn{2}{|c|}{ Zeeman effects } \\
\hline & B. S. & K. \& C. & & & Observed & Computed \\
\hline $\begin{array}{l}50.24 \\
44.36 \\
43.92\end{array}$ & $\begin{array}{l}2 \\
4 \\
5\end{array}$ & 12, III A & $\begin{array}{l}088.22 \\
122.40 \\
124.96\end{array}$ & $\begin{array}{l}a^{2} \mathrm{~F}_{31 / 2}-r^{2} \mathrm{~F}_{31 / 2}^{\circ} \\
a^{4} \mathrm{~F}_{21 / 2}-y^{2} \mathrm{G}_{31 / 2}^{0} \\
a^{4} \mathrm{~F}_{31 / 2}-y^{2} \mathrm{G}_{41 / 2}^{0}\end{array}$ & t & \\
\hline $\begin{array}{l}37.05 \\
17.67\end{array}$ & $\begin{array}{r}20 \\
8\end{array}$ & $\begin{array}{l}40, \text { I } \\
20, \text { III }\end{array}$ & $\begin{array}{l}165.02 \\
278.75\end{array}$ & & $\begin{array}{l}(0.00) 1.22 \\
(0.00) 0.93\end{array}$ & $(0.09) 1.23$ \\
\hline $\begin{array}{l}09.80 \\
09.48\end{array}$ & $\begin{array}{r}10 \\
6\end{array}$ & $\begin{array}{l}20, \text { I A } \\
15, \text { II A }\end{array}$ & $\begin{array}{l}325.24 \\
327.14\end{array}$ & $\begin{array}{l}a^{2} \mathrm{D}_{21 / 2}-v^{2} \mathrm{~F}_{31 / 2}^{\circ} \\
a^{2} \mathrm{D}_{21 / 3}-x^{4} \mathrm{~F}_{31 / 3}^{0}\end{array}$ & $\begin{array}{l}(0.00) 1.04 \\
(0.00) 1.26\end{array}$ & $\begin{array}{l}(0.00) 1.04 \\
(0.00) 1.22\end{array}$ \\
\hline $4,104.87$ & 30 & $60, \mathrm{I}$ & 354.46 & $a^{4} \mathrm{~F}_{11 / 2}-y^{1} \mathrm{G}_{21 / 2}^{2 / 2}$ & $\begin{array}{l}(0.08,0.25) 0.53,0.69 \\
0.85 \text { ur }\end{array}$ & $\begin{array}{l}(0.07,0.23) 0.34,0.49 \text {, } \\
0.64,0.80\end{array}$ \\
\hline $\begin{array}{r}4,090.40 \\
89.61 \\
79.17 \\
65.58 \\
64.79\end{array}$ & $\begin{array}{r}2 \\
25 \\
20 \\
15 \\
25\end{array}$ & $\begin{array}{l}50, \text { I } \\
40, \text { I } \\
30, \text { II } \\
50, \text { II }\end{array}$ & $\begin{array}{l}440.61 \\
445.33 \\
507.90 \\
589.82 \\
594.60\end{array}$ & $\begin{array}{l}a^{2} \mathrm{~F}_{31 / 2}-u^{2} \mathrm{D}_{21 / 2}^{\circ} \\
a^{4} \mathrm{~F}_{21 / 2}-y^{4} \mathrm{G}_{31 / 2}^{0} \\
a^{2} \mathrm{D}_{11 / 2}-v^{2} \mathrm{~F}_{21 / 2}^{21 / 2} \\
a^{2} \mathrm{D}_{21 / 2}-y^{4} \mathrm{P}_{11 / 2}^{0} \\
a^{4} \mathrm{~F}_{31 / 2}-y^{4} \mathrm{G}_{11 / 2}^{\circ}\end{array}$ & $\begin{array}{l}(0.00) 0.93 \\
(0.00 \mathrm{w}) 1.03 \mathrm{~A}^{1}\end{array}$ & $\begin{array}{l}(0.00) 0.94 \\
(0.00) 0.94 \mathrm{~A}^{1}\end{array}$ \\
\hline $\begin{array}{l}60.33 \\
40.97\end{array}$ & $\begin{array}{r}30 \\
2\end{array}$ & 60, II & $\begin{array}{l}621.61 \\
739.57\end{array}$ & $\begin{array}{l}a^{4} \mathrm{~F}_{41 / 3}-y^{4} \mathrm{G}_{51 / 2}^{\circ} \\
a^{2} \mathrm{~F}_{21 / 3}-u^{2} \mathrm{D}_{11 / 2}^{1}\end{array}$ & $(0.00 \mathrm{w}) 1.10 \mathrm{~A}^{1}$ & $(0.00) 1.12 \mathrm{~A}^{1}$ \\
\hline $\begin{array}{l}37.21 \\
15.39\end{array}$ & $\begin{array}{l}25 \\
25\end{array}$ & $\begin{array}{l}50, \mathrm{I} \\
50, \mathrm{I}\end{array}$ & $\begin{array}{l}\text { 762. ô } \\
897.17\end{array}$ & $\begin{array}{l}a^{2} \mathrm{D}_{11 / 2}-x^{2} \mathrm{D}_{21 / 2}^{0} \\
a^{2} \mathrm{D}_{21 / 2}-x^{2} \mathrm{P}_{21 / 2}^{2}\end{array}$ & $\begin{array}{l}(0.00) 0.86 \\
(0.10,0.31) 0.90,1.10 \\
1.30,1.48 \text { ur }\end{array}$ & $\begin{array}{l}(0.13) 0.84 \\
(0.10,0.30) 0.90,1.10 \text {, } \\
\quad 1.30,1.51\end{array}$ \\
\hline $4,001.38$ & 2 & & $24,984.34$ & $a^{2} \mathrm{D}_{11 / 2}-x^{4} \mathrm{~F}_{21 / 5}^{0}$ & & \\
\hline $\begin{array}{r}3,953.67 \\
27.56 \\
3,902.57 \\
3,898.60 \\
3,895.65\end{array}$ & $\begin{array}{r}10 \\
30 \\
5 \\
8\end{array}$ & $\begin{array}{l}\text { 40, II } \\
80, \text { I } \\
20, \text { II } \\
40, \text { II } \\
8 ?, \text { IV }\end{array}$ & $\begin{array}{r}25,285.83 \\
453.92 \\
616.91 \\
25,642.99 \\
25,662.42\end{array}$ & $\begin{array}{l}a^{2} \mathrm{D}_{21 / 2}-y^{4} \mathrm{P}_{31 / 2}^{0} \\
a^{2} \mathrm{D}_{11 / 2}-x^{2} \mathrm{P}_{01 / 2}^{0} \\
a^{2} \mathrm{D}_{13 / 2}-y^{4} \mathrm{P}_{01 / 2}^{013} \\
a^{2} \mathrm{D}_{11 / 2}-y^{4} \mathrm{P}_{11 / 2}^{0}\end{array}$ & $(0.00) 0.76$ & $(0.00) 0.76$ \\
\hline $\begin{array}{r}3,714.30 \\
3,704.54 \\
3,699.57 \\
72.02 \\
49.55\end{array}$ & $\begin{array}{r}2 \\
10 \\
4 \\
8 \\
10\end{array}$ & $\begin{array}{l}40, \text { II } \\
12, \text { II A } \\
30, \text { II } \\
40, \text { II }\end{array}$ & $\begin{array}{r}26,915.34 \\
26,986.25 \\
27,022.51 \\
225.24 \\
392.86\end{array}$ & $\begin{array}{l}a^{2} \mathrm{D}_{21 / 2}-w^{2} \mathrm{D}_{11 / 2}^{\circ} \\
a^{2} \mathrm{D}_{21 / 2}-u^{2} \mathrm{~F}_{31 / 2}^{3} \\
a^{2} \mathrm{D}_{11 / 2}-y^{4} \mathrm{G}_{21 / 2} \\
a^{2} \mathrm{D}_{11 / 2}-w^{2} \mathrm{P}_{11 / 2} \\
a^{2} \mathrm{D}_{11 / 2}-u^{2} \mathrm{~F}_{21 / 2}^{1}\end{array}$ & $\begin{array}{l}(0.00) 1.08 \\
(0.00) 1.00\end{array}$ & $\begin{array}{l}(0.00) 1.04 \\
\pi(0.00) 0.98\end{array}$ \\
\hline $\begin{array}{r}41.53 \\
36.67 \\
3,613.08 \\
3,574.43 \\
3,514.07\end{array}$ & $\begin{array}{c}20 ? \\
6 \\
10 \\
20 \\
6\end{array}$ & $\begin{array}{l}100, \text { II } \\
40, \text { III } \\
30, \text { II } \\
50, \text { II } \\
20, \text { II A }\end{array}$ & $\begin{array}{r}453.19 \\
489.88 \\
669.36 \\
27,968.53 \\
28,448.92\end{array}$ & $\begin{array}{l}a^{2} \mathrm{D}_{21 / 2}-w^{2} \mathrm{D}_{21 / 2}^{\circ} \\
a^{2} \mathrm{D}_{21 / 2}-t^{2} \mathrm{~F}_{31 / 2}^{\circ} \\
a^{2} \mathrm{D}_{11 / 2}-t^{2} \mathrm{~F}_{21 / 2}^{\circ} \\
a^{2} \mathrm{D}_{11 / 2}-w^{2} \mathrm{D}_{11 / 2}^{11 / 2} \\
a^{2} \mathrm{D}_{21 / 2}-w^{4} \mathrm{D}_{23 / 2}^{2}\end{array}$ & $\begin{array}{ll}(0.00) & 0.97 \\
(0.00) & 0.83\end{array}$ & $\begin{array}{ll}(0.00) & 0.90 \\
(0.17) & 0.85\end{array}$ \\
\hline $\begin{array}{r}3,480.61 \\
61.18 \\
50.65 \\
3,404.53 \\
3,388.61\end{array}$ & $\begin{array}{r}3 \\
10 \\
5 \\
10 \\
6\end{array}$ & $\begin{array}{l}\text { 8, III A } \\
25 \text {, III A } \\
12 \text {, III A } \\
15 \text {, III A } \\
12 \text {, II A }\end{array}$ & $\begin{array}{r}722.40 \\
883.64 \\
28,971.78 \\
29,364.24 \\
502.18\end{array}$ & $\begin{array}{l}a^{2} \mathrm{D}_{21 / 2}-v^{2} \mathrm{D}_{21 / 2}^{\circ} \\
a^{2} \mathrm{D}_{21 / 2}-7_{13 / 2}^{\circ} \\
a^{2} \mathrm{D}_{11 / 2}-v^{2} \mathrm{D}_{11 / 2}^{\circ} \\
a^{2} \mathrm{D}_{21 / 2}-8_{11 / 2}^{0} \\
a^{2} \mathrm{D}_{11 / 2}-w^{1} \mathrm{D}_{21 / 2}^{\circ}\end{array}$ & & \\
\hline $\begin{array}{l}81.42 \\
68.36 \\
64.88 \\
62.04 \\
\mathbf{5 7 . 5 0}\end{array}$ & $\begin{array}{l}3 \\
2 \\
7 \\
5\end{array}$ & $\begin{array}{l}\text { 15, II A } \\
\text { 12, III A } \\
7 \text {, III A }\end{array}$ & $\begin{array}{l}564.92 \\
679.54 \\
710.24 \\
735.33 \\
775.54\end{array}$ & $\begin{array}{l}a^{2} \mathrm{D}_{11 / 2}-6_{03 / 2}^{\circ} \\
a^{2}{ }^{2} 31 / 2-11^{\circ} \\
a^{2} \mathrm{~F}_{31 / 2}-10^{\circ} \\
a^{2} \mathrm{D}_{21 / 2}-s^{2} \mathrm{~F}_{21 / 2} \\
a^{2} \mathrm{D}_{13 / 2}-v^{2} \mathrm{D}_{21 / 2}^{2}\end{array}$ & & \\
\hline $\begin{array}{r}49.82 \\
3,342.23 \\
3,256.60 \\
47.06 \\
35.66\end{array}$ & $\begin{array}{r}2 \\
10 \\
2 \\
5 \\
3\end{array}$ & $\begin{array}{l}\text { 3, III A } \\
20, \text { II A } \\
\text { 8, II A } \\
5 \text {, III A }\end{array}$ & $\begin{array}{r}843.80 \\
29,911.57 \\
30,698.05 \\
788.24 \\
30,896.71\end{array}$ & $\begin{array}{l}a^{2} \mathrm{D}_{21 / 2}-9_{21 / 2}^{\circ} \\
a^{2} \mathrm{D}_{21 / 2}-s^{2} \mathrm{~F}_{31 / 2}^{\circ} \\
a^{2} \mathrm{D}_{11 / 2}-s^{2} \mathrm{~F}_{21 / 2}^{\circ} \\
a^{2} \mathrm{D}_{11 / 2}-9_{21 / 3}^{\circ}\end{array}$ & $(0.00) 1.08$ & $(0.00) 1.14$ \\
\hline $\begin{array}{r}3,215.81 \\
3,179.78 \\
75.99 \\
48.51 \\
3,109.42\end{array}$ & $\begin{array}{r}10 \\
4 \\
8 \\
4 \\
8\end{array}$ & $\begin{array}{l}\text { 15, II A } \\
\text { 8, III A } \\
15 \text {, II A } \\
\text { 4, III A } \\
12 \text {, II A }\end{array}$ & $\begin{array}{r}31,087.42 \\
439.65 \\
477.17 \\
31,751.89 \\
32,151.04\end{array}$ & $\begin{array}{l}a^{2} \mathrm{D}_{21 / 2}-r^{2} \mathrm{~F}_{31 / 2}^{0} \\
a^{2} \mathrm{D}_{21 / 2}-u^{2} \mathrm{D}_{21 / 2}^{2} \\
a^{2} \mathrm{D}_{11 / 2}-r^{2} \mathrm{~F}_{21 / 2}^{12} \\
a^{2} \mathrm{D}_{11 / 2}-u^{2} \mathrm{D}_{11 / 2}^{13} \\
a^{2} \mathrm{D}_{21 / 2}-v^{2} \mathrm{P}_{11 / 2}\end{array}$ & (0.00) 1.13 & $(0.00) 1.14$ \\
\hline $\begin{array}{r}3,096.02 \\
10.78 \\
3,001.41 \\
2,992.99 \\
09.65\end{array}$ & $\begin{array}{l}8 \\
4 \\
2 \\
2 \\
2\end{array}$ & 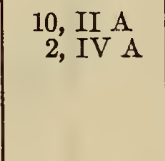 & $\begin{array}{r}32,290.19 \\
33,204.34 \\
307.99 \\
33,401.69 \\
34,358.36\end{array}$ & $\begin{array}{l}a^{2} \mathrm{D}_{11 / 2}-v^{2} \mathrm{P}_{01 / 2}^{0} \\
a^{2} \mathrm{D}_{11 / 2}-v^{2} \mathrm{P}_{11 / 2}^{1}\end{array}$ & & \\
\hline $\begin{array}{r}2,904.62 \\
2,817.46 \\
2,794.03 \\
66.46 \\
61.56\end{array}$ & $\begin{array}{l}1 \\
2 \\
4 \\
4 \\
7\end{array}$ & & $\begin{array}{r}34,417.85 \\
35,482.54 \\
35,780.07 \\
36,136.62 \\
200.74\end{array}$ & $\begin{array}{l}a^{4} \mathrm{~F}_{21 / 2}-13^{\circ} \\
a^{4} \mathrm{~F}_{1 / 2}-14^{\circ}\end{array}$ & & \\
\hline
\end{tabular}


TABLE 6.-The arc spectrum of lanthanum ( $L a I)$-Continued

\begin{tabular}{|c|c|c|c|c|c|c|}
\hline \multirow{2}{*}{$\lambda_{\text {air }}$ I. A. } & \multicolumn{2}{|c|}{$\begin{array}{l}\text { Intensities, tem- } \\
\text { perature class }\end{array}$} & \multirow{2}{*}{$\nu$ vac $\mathrm{cm}^{-1}$} & \multirow{2}{*}{$\begin{array}{l}\text { Term combi- } \\
\text { nation }\end{array}$} & \multicolumn{2}{|c|}{ Zecman effects } \\
\hline & B. S. & K. \& C. & & & Observed & Computed \\
\hline $\begin{array}{l}59.54 \\
56.57 \\
49.52 \\
39.25 \\
37.49\end{array}$ & $\begin{array}{l}4 \\
2 \\
2 \\
4 \\
3\end{array}$ & & $\begin{array}{l}227.24 \\
266.27 \\
359.25 \\
495.56 \\
519.03\end{array}$ & & & \\
\hline $\begin{array}{l}30.15 \\
29.85 \\
25.57 \\
22.31 \\
17.33\end{array}$ & $\begin{array}{r}2 \\
5 \\
15 \\
6 \\
2\end{array}$ & & $\begin{array}{l}617.20 \\
621.22 \\
678.73 \\
722.65 \\
789.90\end{array}$ & $\begin{array}{l}a^{4} \mathrm{~F}_{21 / 2}-13^{\circ} \\
a^{2} \mathrm{D}_{21 / 2}-11^{\circ} \\
a^{2} \mathrm{D}_{11 / 2}-10^{\circ}\end{array}$ & & \\
\hline $\begin{array}{r}15.77 \\
14.52 \\
10.69 \\
2,707.07 \\
2,684.11\end{array}$ & $\begin{array}{l}3 \\
8 \\
4 \\
3 \\
6\end{array}$ & 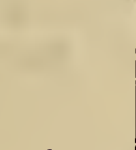 & $\begin{array}{r}811.08 \\
828.03 \\
880.06 \\
36,929.38 \\
37,245.25\end{array}$ & $\begin{array}{l}a^{4} \mathrm{~F}_{31 / 2}-14^{\circ} \\
a^{4} \mathrm{~F}_{11 / 2}-12^{\circ}\end{array}$ & & . \\
\hline $\begin{array}{l}77.77 \\
71.91 \\
53.48 \\
47.13\end{array}$ & $\begin{array}{l}2 \\
2 \\
2 \\
2\end{array}$ & & $\begin{array}{l}333.43 \\
415.30 \\
675.16 \\
765.53\end{array}$ & $\begin{array}{l}a^{4} \mathrm{~F}_{21 / 2}-15^{\circ} \\
a^{4} \mathrm{~F}_{1 / 1 / 2}-15^{\circ}\end{array}$ & & \\
\hline
\end{tabular}

\section{IONIZATION POTENTIALS OF LANTHANUM ATOMS}

The first two members of series have been identified in the spectra of lanthanum in all three degrees of ionization, and the ionization potentials for three different lanthanum atoms, $\mathrm{La} \mathrm{La}^{+}, \mathrm{La}^{++}$, may thus be deduced spectroscopically from extrapolation of the series to their limits.

To use the Rydberg formula is equivalent to assuming that the denominator $n^{*}$ changes by exactly 1 from one term to the next in a series. In those spectra for which long series and accurate limits are available, the change $\Delta n^{*}$ between the first and second members is usually somewhat greater and it appears to be preferable to assume that the value for lanthanum is comparable with that for similar spectra. For example, in the 1-electron spectra we find the following values of $\Delta n$ * for the $s$ electron:

\begin{tabular}{|c|c|c|c|c|c|}
\hline K I & Ca II & Rb I & Sr II & Cs I & Ba II \\
\hline 1.031 & 1.033 & 1.040 & 1.042 & 1.050 & 1.052 \\
\hline
\end{tabular}

We may therefore assume $\Delta n^{*}=1.050$ for La III. The terms $6{ }^{2} \mathrm{~S}$ and $7^{2} \mathrm{~S}$ then give a limit at 154,630 (above the lowest level ${ }^{2} \mathrm{D}_{11 / 2}$ ) with $n^{*}=2.6462,3.6962$. This corresponds to an ionization potential of 19.07 volts which is the lowest known value for a third ionization. Badami ${ }^{26}$ from his analysis of this spectrum derived 20.4 volts for the ionization potential of $\mathrm{La}^{++}$. 
For 2-electron spectra we find for the ${ }^{3} \mathrm{~S}$ and ${ }^{3} \mathrm{D}$ terms due to "running" $s$ and $d$ electrons:

\begin{tabular}{|c|c|c|c|c|c|c|}
\hline \multicolumn{1}{|c|}{ Term } & \multicolumn{3}{|c|}{${ }^{3 \mathrm{~S}}$} & \multicolumn{3}{|c|}{${ }^{3 \mathrm{D}}$} \\
\hline Spectrum & $\mathrm{Ca}$ & $\mathrm{Sr}$ I & Ba I & $\mathrm{Ca}$ I & Sr I & Ba I \\
\hline$\Delta n^{*}-\ldots$ & 1.040 & 1.042 & 1.045 & 1.135 & 1.181 & 1.289 \\
\hline
\end{tabular}

The ${ }^{1} \mathrm{~S}$ and ${ }^{1} \mathrm{D}$ series in the alkaline earths are too much perturbed ${ }^{27}$ to be of use. We therefore assume that in La II $\Delta n^{*}=1.05$ for a running $s$ electron; that is, for the terms $a^{3} \mathrm{D}, f^{3} \mathrm{D}$, and $a^{1} \mathrm{D}, f^{1} \mathrm{D}$, with limit $5^{2} \mathrm{D}$ in La III, and 1.15 for a running $d$ electron $\left(a^{3} \mathrm{D}, h^{3} \mathrm{D}\right.$ with limit $6^{2} \mathrm{~S}$ ). The resulting values of $n^{*}$ (referred to the proper limit for each component) and of the difference between the lowest level in. La II $\left(a^{3} \mathrm{~F}_{2}\right)$ and La III $\left(5^{2} \mathrm{D}_{11 / 2}\right)$ are as follows:

\begin{tabular}{|c|c|c|c|c|c|c|c|}
\hline Term & ${ }^{3} \mathrm{D}_{1}$ & ${ }^{3} \mathrm{D}_{2}$ & ${ }^{3} \mathrm{D}_{3}$ & ${ }^{1} \mathrm{D}_{1}$ & ${ }^{3} \mathrm{D}_{1}$ & ${ }^{3} \mathrm{D}_{2}$ & ${ }^{3} \mathrm{D}_{3}$ \\
\hline $\begin{array}{l}n^{*} \text {--.--- } \\
\text { Limit }\end{array}$ & $\left\{\begin{array}{l}2,2232 \\
3,2732 \\
90,704\end{array}\right.$ & $\begin{array}{l}2,2333 \\
3,2833 \\
90,600\end{array}$ & $\begin{array}{l}2,2206 \\
3,2706 \\
90,664\end{array}$ & $\begin{array}{l}2,1826 \\
3,2326 \\
91,924\end{array}$ & $\begin{array}{l}2,0384 \\
3,1884 \\
93,944\end{array}$ & $\begin{array}{l}2,0453 \\
3,1953 \\
93,924\end{array}$ & $\begin{array}{l}2,0518 \\
3,2018 \\
93,916\end{array}$ \\
\hline
\end{tabular}

The mean of these seven values is 92,240 , which corresponds to an ionization potential of 11.38 volts for $\mathrm{La}^{+}$.

Finally, for La I, we have the terms $a^{4} \mathrm{~F}, e^{4} \mathrm{~F}$ and $a^{2} \mathrm{~F}, e^{2} \mathrm{~F}$ with a running $s$ electron and limit $a^{3} \mathrm{~F}$ of La II. With limit $a^{3} \mathrm{D}$ we have only $a^{2} \mathrm{D}$ and $e^{4} \mathrm{D}$, but these terms, though of different multiplicity, may still be used. ${ }^{28}$ The first comes from the configuration $5 d 6 s^{2}$, the second from $5 d 6 s 7 s$. Dropping the $5 d$ electron we obtain the lowest ${ }^{1} \mathrm{~S}$ and ${ }^{3} \mathrm{~S}$ terms in $\mathrm{Ba} \mathrm{I}$. For these and the corresponding terms in Ca I and Sr I we have the values of $n^{*}$.

\begin{tabular}{|c|c|c|c|}
\hline Spectrum & $\mathrm{Ca} \mathrm{I}$ & $\mathrm{Sr} \mathrm{I}$ & $\mathrm{Ba} \mathrm{I}$ \\
\hline$n^{*}\left({ }^{1} \boldsymbol{S}\right)$ & 1. 442 & 1.545 & 1.616 \\
\hline $\begin{array}{l}n^{*}(3 \mathrm{~S}) \\
\Delta n^{*}\end{array}$ & $\begin{array}{l}2.484 \\
1.042\end{array}$ & $\begin{array}{l}2.548 \\
1.003\end{array}$ & $\begin{array}{l}2.629 \\
1.013\end{array}$ \\
\hline
\end{tabular}

We may, therefore, adopt $\Delta n^{*}=1.010$ in this case while in the others we use 1.060. We then find

\begin{tabular}{|c|c|c|c|c|c|c|c|}
\hline Term & $\begin{array}{l}a^{2} \mathrm{D}_{21 / 3} \\
e^{4} \mathrm{D}_{31 / 6}\end{array}$ & $\begin{array}{l}a^{4} \mathrm{~F}_{13 / 5} \\
d^{4} \mathrm{~F}_{11 / 6}\end{array}$ & $\begin{array}{l}a^{4} \mathrm{~F}_{21 / 2} \\
e^{4} \mathrm{~F}_{21 / 2}\end{array}$ & $\begin{array}{l}a^{4} \mathrm{~F}_{31 / 2} \\
e^{4} \mathrm{~F}_{3 / 2}\end{array}$ & $\begin{array}{l}a^{4} \mathrm{~F}_{43 / 2} \\
e^{4} \mathrm{~F}_{43 / 6}\end{array}$ & $\begin{array}{l}a^{2} \mathrm{~F}_{23 / 5} \\
e^{2} \mathrm{~F}_{21 / 2}\end{array}$ & $\begin{array}{l}a^{2} \mathrm{~F}_{33 / 4} \\
e^{2} \mathrm{~F}_{31 / 5}\end{array}$ \\
\hline & $\left\{\begin{array}{l}1.5221 \\
2.5321\end{array}\right.$ & $\begin{array}{l}\text { 1. } 6033 \\
\text { 2. } 6633\end{array}$ & $\begin{array}{l}1.6000 \\
2.6600\end{array}$ & 1. 5948 & 1. 5892 & $\begin{array}{l}\text { 1. } 6829 \\
\text { 2. } 7429\end{array}$ & $\begin{array}{l}\text { 1. } 6855 \\
\text { 2. } 7455\end{array}$ \\
\hline Limit_- & 45,145 & 45,337 & 45,856 & 45,606 & 45,583 & 44,721 & 44,687 \\
\hline
\end{tabular}

The mean is 45,293 for the interval from $a^{2} \mathrm{D}_{11 / 2}$ (La I) to $a^{3} \mathrm{~F}_{2}$ (La II). This corresponds to an ionization potential of 5.59 volts.

A change of +0.01 in $\Delta n^{*}$ alters the computed limits on the average by -152 or -0.019 volts. The average discordance of the seven

${ }_{27}$ A. G. Shenstone and H. N. Russell, Phys. Rev., vol. 39, p. 415, 1932.

28 We owe this suggestion to Prof. A. G. Shenstone. 
determinations from the mean is \pm 334 or \pm 0.041 volts. We, therefore, adopt $5.59 \pm 0.03$ volts as the first ionization potential of lanthanum. This is in excellent agreement with the value 5.49 volts derived by Rolla and Piccardi ${ }^{29}$ from observations of the ionization current and rate of dissociation of $\mathrm{La}_{2} \mathrm{O}_{3}$ in flames.

For La II a change in $\Delta n^{*}$ by \pm 0.01 affects the ionization potential by \pm 0.044 volts, while the average discordance of one determination is \pm 0.13 volts.

We may finally adopt for lanthanum atoms:

First ionization potential $=5.59 \pm 0.03$ volts.
Second ionization potential $=11.38 \pm 0.07$ volts.
Third ionization potential $=19.1 \pm 0.1$ volts.

\section{DISCUSSION OF ZEEMAN EFFECTS}

The observed Zeeman effects for lanthanum lines in all three spectra positively identify most of the spectral terms although a comparison of the observed and theoretical $g$ values discloses many discrepancies which greatly exceed the experimental errors. Similar deviations have been observed in other spectra, and have been assigned either to departures from strict SL coupling, or to the sharing of $g$ values by mutually perturbing terms. If the coupling between spin moment and orbital moment of each electron is weak individually as compared with the coupling of the spins and those of the orbital moments of the electrons mutually we have the so-called SL coupling:

$$
\left\{\left(s_{1} s_{2 \ldots} \ldots\right)\left(l_{1} l_{2-\ldots}\right)\right\}=(S L)=J
$$

An atom for which this is true will emit a spectrum in which interval rules, and Zeeman effects are in accord with the values predicted by the theory of Landé and others. But if the quantum vectors $s$ and $l$ are actually compounded to produce a resultant vector $J$ in some other manner the spectrum will show violations of the interval rules and deviations from Landé's $g$ values. Such deviations are evident in the La spectra. For example, the metastable quartet- $F$ term in the La I spectrum shows only a slight departure from the interval rule:

\begin{tabular}{|c|c|c|}
\hline & Separations & Ratios \\
\hline $\begin{array}{l}\text { Theoretical } \\
\text { La I }\end{array}$ & 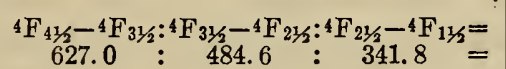 & \begin{tabular}{|l} 
9. $00: 7.00: 5.00$ \\
$9.00: 6.95: 4.90$
\end{tabular} \\
\hline
\end{tabular}

and the observed $g$ values are almost exactly those of Landé

\begin{tabular}{|c|c|c|}
\hline Level & Observed $g$ & Landé $g$ \\
\hline $\begin{array}{l}{ }^{4} \mathrm{~F}_{13 / 2} \\
{ }^{4} \mathrm{~F}_{21 / 3} \\
{ }^{4} \mathrm{~F}_{31 / 2} \\
{ }^{4} \mathrm{~F}_{41 / 2}\end{array}$ & $\begin{array}{l}0.411 \\
1.032 \\
1.222 \\
1.337\end{array}$ & $\begin{array}{l}0.400 \\
1.029 \\
1.238 \\
1.333\end{array}$ \\
\hline
\end{tabular}

The low terms in the La II spectrum do greater violence to the interval rule, and also somewhat larger divergences of observed and theoretical

${ }^{29}$ L. Rolla and G. Piccardi, Phil. Mag., vol. 7, p. 286, 1929. 
$g$ values occur. Thus, for the normal state represented by $a^{3} \mathrm{~F}$ we have the following interval ratios:

\begin{tabular}{|c|c|c|}
\hline & Separations & Ratios \\
\cline { 2 - 3 } Theoretical & & \\
\hline La II & & \\
\hline
\end{tabular}

and the following $g$ values:

\begin{tabular}{|c|c|c|}
\hline Level & $\begin{array}{c}\text { Observed } \\
g\end{array}$ & Lanđé $g$ \\
\hline${ }^{3} \mathrm{~F}_{2}$ & 0.730 & 0.667 \\
$3 \mathrm{~F}_{3}$ & 1.092 & 1.083 \\
${ }^{3 \mathrm{~F}_{4}}$ & 1.258 & 1.250 \\
\hline
\end{tabular}

Similarly for the term $a^{3} \mathrm{D}$, the interval ratios are:

\begin{tabular}{|c|c|c|}
\hline & Separations & Ratios \\
\hline $\begin{array}{l}\text { Theoretical } \\
\text { La II }\end{array}$ & $\begin{array}{c}{ }^{3} \mathrm{D}_{3}{ }^{-3} \mathrm{D}_{2}:{ }^{3} \mathrm{D}_{2}-3 \mathrm{D}_{1} \\
658.8: 696.4\end{array}$ & $\begin{array}{l}\text { 3. } 00: 2.00 \\
3.00: 3.17\end{array}$ \\
\hline
\end{tabular}

and the splitting factors are:

\begin{tabular}{|c|c|c|}
\hline Level & $\underset{g}{\text { Observed }}$ & Landé \\
\hline${ }^{3} \mathrm{D}_{1}$ & $\begin{array}{l}0.520 \\
1.140\end{array}$ & $\begin{array}{l}0.500 \\
1.167\end{array}$ \\
\hline $\begin{array}{l}{ }^{3} D_{2} \\
{ }_{3}\end{array}$ & $\begin{array}{l}1.140 \\
1.337\end{array}$ & $\begin{array}{l}1.107 \\
1.333\end{array}$ \\
\hline
\end{tabular}

These, and many other departures from theoretical values indicate a considerable deviation from (SL) coupling, probably in the direction of $(j j)$ coupling defined, in the case of two electrons, by

$$
\left\{\left(s_{1} l_{1}\right)\left(s_{2} l_{2}\right)\right\}=\left(j_{1} j_{2}\right)=J
$$

in which the interactions between spin and orbital moment of each electron predominate.

No matter what the nature of the coupling may be the so-called $g$-sum rule of Pauli ${ }^{30}$ is expected to be valid. This rule can be stated as follows: If, among all the terms arising through the coupling of one electron, those terms with the same inner quantum number $J$ be grouped, the sum of the $g$ values of the terms in each group must have a value independent of the nature of the coupling, and consequently is, among others, equal to the sum of the Landé $g$ values for a similar group. This rule is tested in Table 7 where the observed and Landé $g$ sums are displayed for all the groups in La II for which the data are complete and for the low terms in La I, although one term has not been found. The sums of observed $g$ values are slightly larger than the theoretical sums, but the differences can perhaps be ascribed to the accumulation of a small systematic error of observation. If the observered $g$ 's are diminished by 0.78 per cent, to allow for this, the agreement becomes almost perfect, with an average discordance of only 0.5 per cent. 


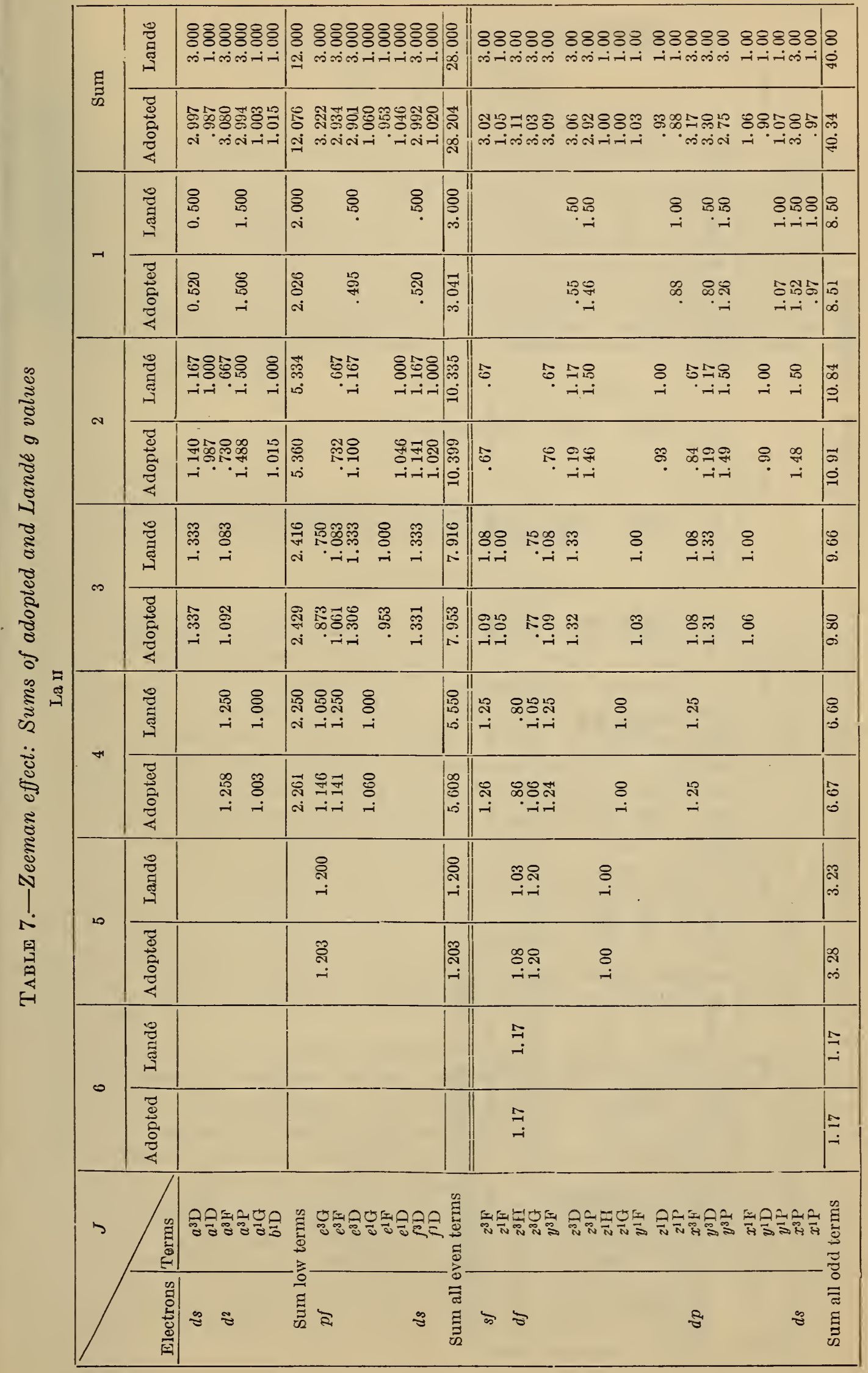




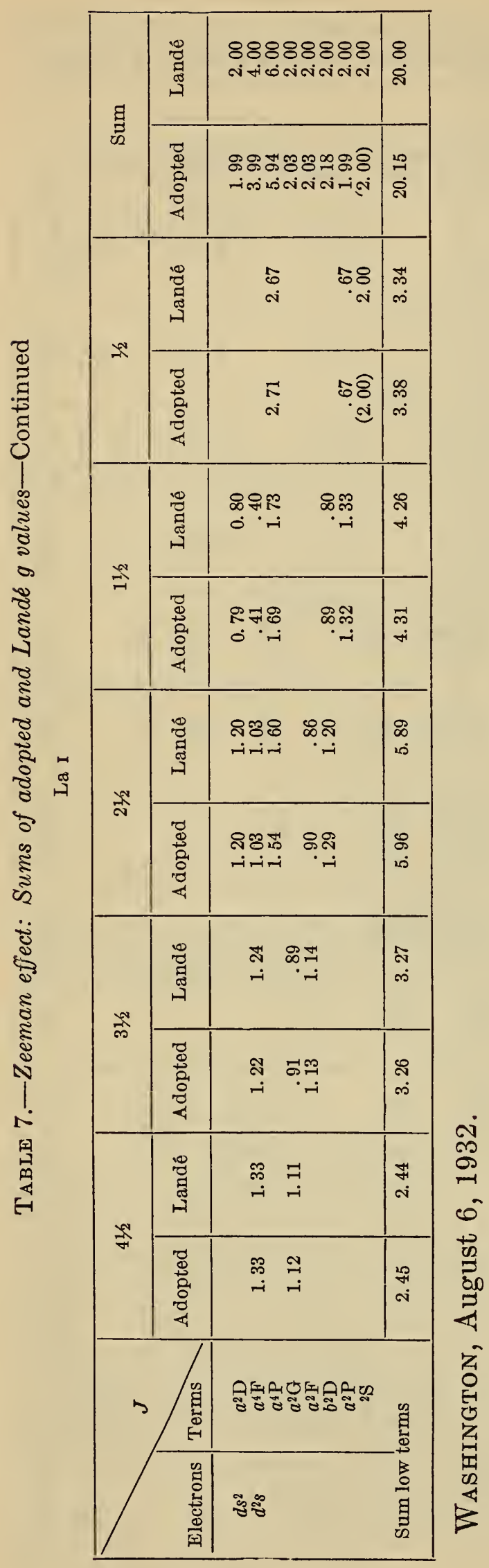

